\author{
Universidade de São Paulo \\ Instituto de Astronomia, Geofísica e Ciências Atmosféricas
}

Departamento de Astronomia

Patricia Martins de Novais

\title{
A Distribuição de Populações Estelares em Galáxias
}

São Paulo 



\section{A Distribuição de Populações Estelares em Galáxias}

Dissertação apresentada ao Departamento de Astronomia do Instituto de Astronomia, Geofísica e Ciências Atmosféricas da Universidade de São Paulo como requisito parcial para a obtenção do título de Mestre em Ciências.

Área de Concentração: Astronomia Orientador: Prof. Dr. Laerte Sodré Jr.

Versão Corrigida. O original encontrase disponível na Unidade.

São Paulo 

(...)pessoas habitadas são aquelas possuídas, de fato, por si mesmas, em diversas versões. Os habitados estão preenchidos de indagações, angústias, incertezas, mas não são menos felizes por causa disso. Não transformam suas inadequações em doença, mas em força e curiosidade. Não recuam diante de encruzilhadas, não se amedrontam com transgressões, não adotam as opiniões dos outros para facilitar o diálogo. São pessoas que surpreendem com um gesto ou uma fala fora do script, sem nenhuma disposição para serem bonecos de ventríloquos. Ao contrário, encantam pela verdade pessoal que defendem. Além disso, mantém com a solidão uma relação mais do que cordial.

Martha Medeiros (in Pessoas Habitadas) 



\section{Agradecimentos}

Gostaria de agradecer, e muito, o apoio da minha família nesse caminho em busca do conhecimento. Aos meus irmãos Anderson, Wellington e Jéssica por me apoiarem e sempre me ajudarem a criar forças para vencer as dificuldades diárias. A minha mãe, minha maior amiga, que foi paciente e carinhosa em todos os mmomentos de desesperos e angústias ao longo do trajeto até aqui. Ao senhor Orlando, meu falecido pai, dedico este trabalho, pois sei que ele ficaria muito orgulhoso de ver tudo o que a sua filha conquistou. Ao Walisson, meu grande amor, agradeço pelo companheirismo, pela força, compreensão e por andar de mãos dadas comigo, não só no caminho profissional como também em todos os outros caminhos!

Ao meu orientador, professor Laerte Sodré Jr., agradeço imensamente não somente pela orientação nesse trabalho, como também por todas as coisas que me ensinou desde meados de 2006, quando me aceitou como aluna de iniciação científica. Um orientador sempre presente e comprometido com um bom trabalho, com o meu crescimento profissional. Sem dúvidas, se hoje eu continuo na academia o mérito é do professor Laerte!

Aos amigos do grupo do professor Laerte: Ana Soja, Ana O’Mill, Ana Carolina, Marcus Duarte... Além de ser um grupo muito divertido, é um grupo muito unido. Sendo assim, muito obrigada pela ajuda e apoio, vocês foram essenciais em diversos momentos, contribuindo muito para o desenvolvimento do presente trabalho.

Ainda, gostaria muito de agradecer aos colegas que me ajudaram direta ou indiretamente, tanto no desenvolvimento do trabalho quanto em questões do dia a dia. Entre esses amigos, gostaria de citar minha grande amiga e companheira de jornada Carla Trucolo, meus amigos Felipe Oliveira, Vinicius Busti, Nathália Cibirka, Daiane Seriacopi, Marcelo Rubinho, Aline Ribeiro, Walter Santos... 
Agradeço também à agência financiadora CAPES (bolsa de mestrado PROEX) e, por último, mas não menos importante, agradeço ao departamento de Astronomia do IAG/USP, as secretárias, os técnicos e todos os que colaboraram para a contrução dessa dissertação.

Esta tese/dissertação foi escrita em $\mathrm{HAT}_{\mathrm{E}} \mathrm{X}$ com a classe IAGTESE, para teses e dissertações do IAG. 
"O correr da vida embrulha tudo. A vida é assim: esquenta e esfria, aperta e daí afrouxa, sossega e depois desinquieta. O que ela quer da gente é coragem."

João Guimarães Rosa

"Nada de imitar seja lá quem for. Temos de ser nós mesmos. Ser núcleo de cometa, não cauda. Puxar fila, não seguir."

Monteiro Lobato 



\section{Resumo}

O estudo de populações estelares em galáxias é particularmente interessante, uma vez que são um registro fóssil de vários processos físicos associados com a formação e evolução das galáxias. Neste trabalho são apresentados os resultados preliminares de uma nova abordagem para o estudo da distribuição de populações estelare no interior das galáxias.

A partir das magnitudes ugriz de uma amostra de galáxias, utilizou-se a análise PCA e a determinação de diversos parâmetros para estudar a distribuição espacial das populações estelares nas galáxias. Utilizando uma abordagem píxel a píxel, as populações estelares são investigadas através da aplicação de variadas ferramentas estatísticas, tais como Índices de Gini e Funcionais de Minkowski.

Esta abordagem é um passo a frente no estudo de galáxias, no sentido que a análise pode ser aprofundada em cada píxel da galáxia, ao invés de estudar a galáxia como um todo. A aplicação de tal tratamento aos píxeis de uma imagem permite a obtenção de estimativas quantitativas sobre a forma como as diferentes populações estelares são distribuídos dentro de uma galáxia, trazendo dicas sobre como elas crescem e evoluem.

Nossos resultados preliminares mostraram que uma metodologia píxel a píxel é eficiente no estudo das galáxias. Analisando 15 galáxias de tipos distintos, observou-se que as mesmas possuem populações estelares velhas no centro e novas nas regiões periféricas, corroborando com o cenário inside-out de formação de galáxias. Dada a potencialidade do método desenvolvido, pretende-se automatiza-lo para aplicação em dados de grandes surveys. 



\section{Abstract}

The study of stellar populations in galaxies is particularly interesting since they are a fossil record of various physical processes associated with the formation and evolution of galaxies. This work presents the preliminary results of a new approach to the study of the spatial distribution of stellar populations within a galaxy.

From the ugriz magnitudes of a sample of galaxies, we used the PCA analysis and determination of various parameters to study the spatial distribution of the stellar populations in galaxies. Using a pixel by pixel approach, the stellar populations are investigated through the application of various statistical tools, such as Gini indexes and Minkowski Functional.

This approach is a step forward in the study of galaxies, in the sense that the analysis can be deepened in each pixel of the galaxy, rather than studying the galaxy as a whole. The application of such treatment to the pixels of an image allows to obtain quantitative estimates on how the different stellar populations are distributed within a galaxy, bringing tips on how they grow and evolve.

Our preliminary results showed that a pixel by pixel approach is efficient in the study of galaxies. Analyzing 15 galaxies of different types, we observed that they have old stellar populations in the central regions and younger stellar population in the peripheral regions, corroborating the scenario inside-out formation of galaxies. Given the potential of the developed method, the aim is automate and to apply it in data of large surveys. 



\section{Lista de Figuras}

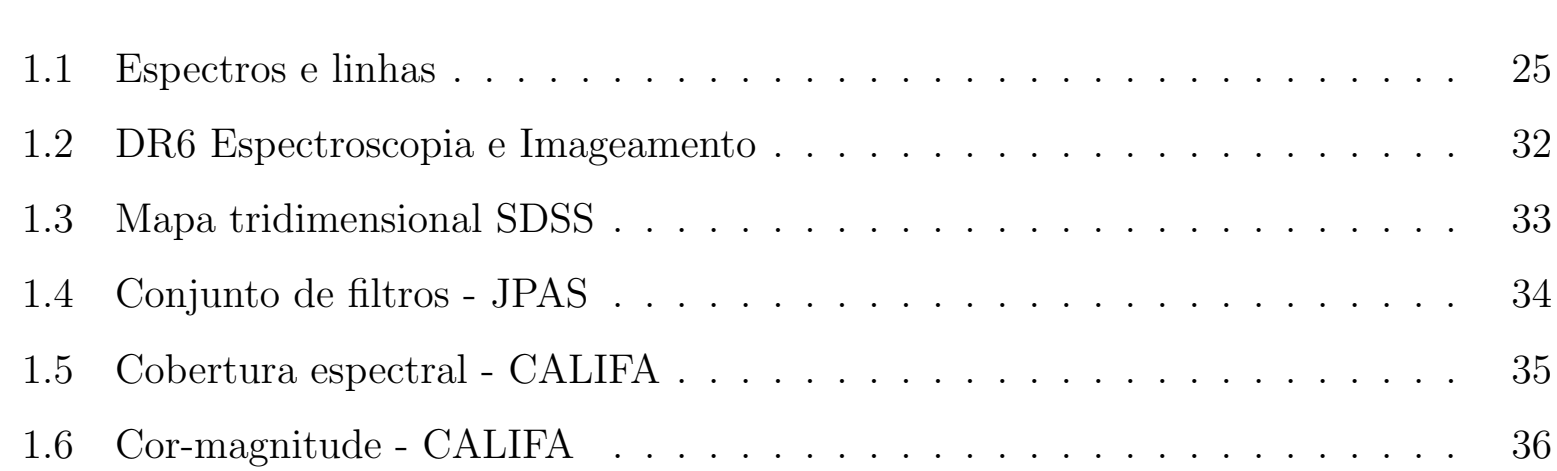

2.1 Amostra de dados: Early-tvpes . . . . . . . . . . . . . . . . . . 41

2.2 Amostra de dados: Late-tvpes . . . . . . . . . . . . . . . . . . . . 42

2.3 Amostra de dados: Late-types . . . . . . . . . . . . . . . . . . . . . . 42

2.4 Amostra de dados - SDSS $\ldots \ldots \ldots \ldots \ldots$

2.5 Amostra de dados - SDSS $\ldots \ldots \ldots \ldots$

3.1 PCA do SDSS . . . . . . . . . . . . . . . . . . . . . . . . . 49

3.2 Galáxias do SDSS: Primeira componente principal versus cores . . . . . . . 50

3.3 PCA do SDSS - Idade e Metalicidade . . . . . . . . . . . . . . . 50

3.4 Exemplo de resultado: PCA. . . . . . . . . . . . . . . . 52

3.5 Exemplo de resultado: PCA1 vs PCA2. . . . . . . . . . . . . . . . . 53

3.6 Exemplo de resultado: PCA1 vs cores . . . . . . . . . . . . . . . . . . . 53

$4.1 \mathrm{NGC2916} \ldots \ldots \ldots \ldots \ldots \ldots \ldots$

4.2 NGC2916 SDSS . . . . . . . . . . . . . . . . . . . . . . . . 56

4.3 NGC2916 SDSS . . . . . . . . . . . . . . . . . . . . . . . . 57

4.4 Mapa de Segmentacão $\ldots$. . . . . . . . . . . . . . . . . . . . . 60

4.5 Mapa Friends of Friends . . . . . . . . . . . . . . . . . . . . . . . . . . . . . 61 
4.6 Mapa de Distribuição espacial de cor . . . . . . . . . . . . . . . . . . . . 62

6.1 Exemplo de resultado: PCA1 vs cores . . . . . . . . . . . . . . . 70

6.2 Exemplo de resultado: PCA1 vs cores . . . . . . . . . . . . . . . 71

6.3 Exemplo de resultado: PCA1 vs cores . . . . . . . . . . . . . . . . 72

6.4 Distribuicão espacial das populacões estelares $\ldots \ldots \ldots \ldots \ldots$

6.5 Distribuicão espacial das populacões estelares $\ldots \ldots \ldots$. . . . . . . . 74

6.6 Distribuicão espacial das populacões estelares $\ldots \ldots \ldots$. . . . . . . 75

6.7 Distribuicão espacial das populações estelares $\ldots \ldots$. . . . . . . . . 76

6.8 Raios médios e cores $\ldots \ldots \ldots \ldots \ldots$

6.9 Coeficiente de Gini . . . . . . . . . . . . . . . . . . . 80

6.10 Característica de Euler-Poincaré . . . . . . . . . . . . . . . . . . 83

A.1 Resultado: PCA1 vs cores . . . . . . . . . . . . . . . . . . . . . . . . 104

A.2 Resultado: PCA1 vs cores . . . . . . . . . . . . . . . . . . . . . . 104

A.3 Resultado: PCA1 vs cores . . . . . . . . . . . . . . . . . . 105

A.4 Resultado: PCA1 vs cores . . . . . . . . . . . . . . . . . . . . 105

A.5 Resultado: PCA1 vs cores . . . . . . . . . . . . . . . . . 106

A.6 Exemplo de resultado: PCA1 vs cores . . . . . . . . . . . . . . . . . 107

A.7 Exemplo de resultado: PCA1 vs cores . . . . . . . . . . . . . . . . . . 107

A.8 Exemplo de resultado: PCA1 vs cores . . . . . . . . . . . . . . . . . . 108

A.9 Exemplo de resultado: PCA1 vs cores . . . . . . . . . . . . . . . . . 108

A.10 Exemplo de resultado: PCA1 vs cores . . . . . . . . . . . . . . . . . . 109

A.11 Exemplo de resultado: PCA1 vs cores . . . . . . . . . . . . . . . . . 110

A.12 Exemplo de resultado: PCA1 vs cores . . . . . . . . . . . . . . . . . . 110

A.13 Exemplo de resultado: PCA1 vs cores . . . . . . . . . . . . . . . . . 111

A.14 Exemplo de resultado: PCA1 vs cores . . . . . . . . . . . . . . . . . . 111

A.15 Exemplo de resultado: PCA1 vs cores . . . . . . . . . . . . . . . . . . 112

A.31 Raios médios e cores . . . . . . . . . . . . . . . . . . . . 121

A.32 Raios médios e cores . . . . . . . . . . . . . . . . . . . . . 122

A.33 Raios médios e cores . . . . . . . . . . . . . . . . . . . . . . . 123

A.34 Coeficiente de Gini - Elípticas . . . . . . . . . . . . . . . . . . . . 124

A.35 Coeficiente de Gini - Espirais . . . . . . . . . . . . . . 125 
A.36 Coeficiente de Gini - Espirais tardias . . . . . . . . . . . . . 126

A.37 Característica de Euler-Poincaré - Espirais tardias . . . . . . . . . . . . . 127

A.38 Característica de Euler-Poincaré - Espirais . . . . . . . . . . . . . . . . 128

A.39 Característica de Euler-Poincaré - Espirais tardias . . . . . . . . . . . . . 129 



\section{Lista de Tabelas}

2.1 Amostra de Galáxias . . . . . . . . . . . . . . . . . . . . . 40

3.1 Componentes Principais do SDSS . . . . . . . . . . . . . . . 48

4.1 Estatísticas do céu da galáxia NGC2916 . . . . . . . . . . . . . . . 58

4.2 Calibração Fotométrica . . . . . . . . . . . . . . . . . . . . . . . . . . . . . 59

6.1 Coeficientes de Gini . . . . . . . . . . . . . . . . . . . . . . . . . . 81

6.2 Funcionais de Minkowski . . . . . . . . . . . . . . . . . . . . . . . . 82 



\section{Sumário}

1. Introducãd . . . . . . . . . . . . . . . . . . . . . . . 21

1.1 Populacões Estelares _. . . . . . . . . . . . . . . . . . . . . 22

1.2 Síntese de Populacões Estelares . . . . . . . . . . . . . . . . . 23

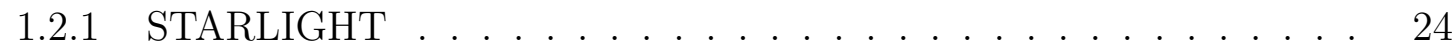

1.3 Distribuicão das Populacões Estelares e suas Propriedades . . . . . . . . . 26

1.3.1 Resultados do CALIFA . . . . . . . . . . . . . . . . . . 30

1.4 Os Grandes Surveys Modernos . . . . . . . . . . . . . . . . . . . . . . . 31

1.4 .1 SDSS/DR7 . . . . . . . . . . . . . . . . . . . 32

1.4 .2 JPAS Survev . . . . . . . . . . . . . . . . . . . 33

1.4 .3 CALIFA Survev . . . . . . . . . . . . . . . . . . . . . 34

1.5 Populações Estelares - Um outro approach . . . . . . . . . . . . . . . . . 36

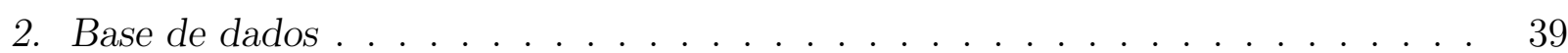

2.1 Amostra de galáxias do CALIFA . . . . . . . . . . . . . . . . . . . . . 39

2.2 Amostra de galáxias do SDSS . . . . . . . . . . . . . . . . . 40

3. Populacões Estelares . . . . . . . . . . . . . . . . . . . . 45

3.1 Análise de Componentes Principais - PCA . . . . . . . . . . . . . . 45

$3.2 \quad \mathrm{PCA}$ do SDSS . . . . . . . . . . . . . . . . . . . . . . . . . 48

3.3 PCA das cores em cada píxel . . . . . . . . . . . . . . . 51

3.4 Conclusões . . . . . . . . . . . . . . . . . . . . . . . . . . 54

4. Análise das Imagens das Galáxias . . . . . . . . . . . . . . . . . . . . . . 55

4.1 Extração, Redução e Pré-Processamento dos Dados . . . . . . . . . . . . 56 


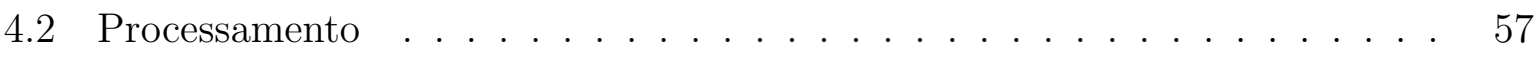

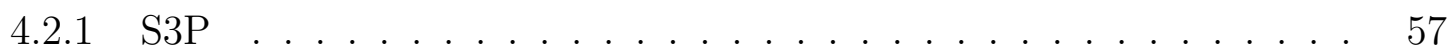

5. Estimadores Estatísticos da Distribuicão de Populações Estelares . . . . . . . . 65

5.1 Raio Médio e Dispersão . . . . . . . . . . . . . . . . . . . . 66

5.2 Coeficiente de Gini . . . . . . . . . . . . . . . . . 66

5.3 Funcionais de Minkowski . . . . . . . . . . . . . . . . . . . . . . 67

6. Resultados. . . . . . . . . . . . . . . . . . . . . . . . . . . . . 69

$6.1 \quad$ PCA e Populacões Estelares Píxel a Píxel . . . . . . . . . . . . . . . . . . 69

6.2 Distribuicãa Espacial de Populações Estelares … . . . . . . . . . . 72

6.3 Cores e Raios Médios . . . . . . . . . . . . . . . . . . . . . . . . 77

6.4 Coeficiente de Gini .... . . . . . . . . . . . . . . . . . . . . . . . . 79

6.5 Funcionais de Minkoswki . . . . . . . . . . . . . . . . . . . . . . 81

7. Conclusões e Perspectivas . . . . . . . . . . . . . . . . . . . . . 85

7.1 Conclusões $\ldots \ldots \ldots \ldots \ldots \ldots \ldots$

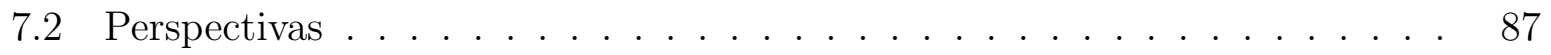

Referências . . . . . . . . . . . . . . . . . . . . 89

$\begin{array}{ll}\text { Apêndice } & 101\end{array}$

A. Apêndice-Resultados . . . . . . . . . . . . . . . . . . . . . . 103

A.1 PCA e Populacões Estelares Píxel a Píxel . . . . . . . . . . . . . . . . . 103

A.1.1 Galáxias Elípticas . . . . . . . . . . . . . . . . . . . . . . . 103

A.1.2 Galáxias Espirais . . . . . . . . . . . . . . . . . . 106

A.1.3 Galáxias Espirais Tardias . . . . . . . . . . . . . . . . . . . . 109

A.2 Distribuicão Espacial . . . . . . . . . . . . . . . . . . . . . . . . . . . . 112

A.2.1 Galáxias Elípticas . . . . . . . . . . . . . . . . . . . . . 113

A.2.2 Galáxias Espirais . . . . . . . . . . . . . . . . . . . 115

A.2.3 Galáxias Espirais Tardias . . . . . . . . . . . . . . . 118

A.3 Cores e Raios Médios . . . . . . . . . . . . . . . . . . . . . . . . . . 120

A.4 Coeficientes de Gini . . . . . . . . . . . . . . . . . . . . . . . . 124 
A.5 Funcionais de Minkowski . . . . . . . . . . . . . . . . . . . . . . 126 

Capítulo 1

\section{Introdução}

O complexo processo pelo qual as galáxias se formam e evoluem é, ainda hoje, um dos principais desafios da Astrofísica e da Cosmologia moderna (Cid Fernandes et al., 2007; Hopkins et al., 2009). Desde a época em que designávamos esses objetos simplesmente como nebulosas até os dias atuais, em que é possível obter imagens e espectroscopia de milhões de galáxias, com os enormes surveys modernos (Abazajian et al., 2009; Colless, 1999), nossa compreensão a respeito desses curiosos objetos tem mudado severamente. Se houve um tempo em que mal sabíamos determinar a sua natureza, hoje conseguimos explorar e estudar diversas de suas características e propriedades, do Grupo Local a altos redshifts (Ownsworth et al., 2012). Muito além dos poucos objetos estudados por Hubble (Hubble, 1920, 1922, 1926, 1929), hoje temos acesso a uma grande diversidade de objetos extragalácticos.

Mais do que descrever as galáxias, é necessário entender como tais objetos são formados e quais os mecanismos responsáveis por imprimir nelas as suas principais características, tanto morfológicas quanto estruturais. É de extrema importância, também, entender o modo pelo qual tais objetos astronômicos evoluem. Assim sendo, um dos principais objetivos do estudo das galáxias é entender a maneira como as populações estelares se formam, distribuem, evoluem e interferem dentro delas.

Uma vez que não é possível resolver individualmente cada estrela numa galáxia, exceto em galáxias muito próximas a nós, tais como as Nuvens de Magalhães e outros objetos do Grupo Local, para que seja possível estudar suas populações estelares, temos que lançar mão de outros observáveis, tais como seus espectros integrados. Através do estudo e análise de espectros integrados é possível entender e explorar características importantes das populações estelares, tais como idade, cinemática, composição química, histórias de formação 
estelar, cores, dentre outros conceitos fundamentais úteis no entendimento da natureza das galáxias (Eggen et al., 1962; Freeman e Bland-Hawthorn, 2002).

O estudo das propriedades das populações estelares é uma ferramenta chave no entendimento e diagnóstico de certas previsões de efeitos evolutivos e para testar modelos teóricos sobre a formação e evolução de galáxias, uma vez que as populações estelares são registros fósseis dos processos que aconteceram nas galáxias (Barker et al., 2007). Diversos estudos, utilizando espectros integrados, foram realizados nos últimos anos levando a uma maior compreensão da natureza desses objetos. Entretanto, com a grande quantidade de surveys que estão em andamento ou sendo construídos e programados para os próximos anos (Ivezic et al., 2009; Sodré, 2012b, a,; Sánchez et al., 2011) é possível ir além do estudo individual das galáxias, o que requer o desenvolvimento de novos métodos de análise.

Ainda que o estudo das populações estelares através do espectro integrado de galáxias como um todo seja uma ferramenta extremamente útil, e que tem se desenvolvido muito ao longos dos anos, tais esforços fornecem apenas informações globais a respeito da galáxia, não trazendo detalhes sobre os processos internos e evoluções seculares na mesma. Dessa forma, é necessário a elaboração de novos métodos e técnicas de análise que levem em conta a dimensão espacial das populações estelares.

\subsection{Populações Estelares}

As estrelas que constituem uma galáxia, em geral, não se formam isoladamente, mas sim em grupos de centenas ou milhares de membros. Essas aglomerações de estrelas possuem propriedades muito características, de modo que é interessante estudarmos o que é compreendido como populações estelares.

Baade (1944) estudou as estrelas da galáxia Andrômeda e os satélites M32 e NGC205, propondo então que as estrelas poderiam ser divididas em duas populações distintas, a saber, População I e População II. No caso da Via Láctea, as estrelas de População I foram classificadas como sendo as estrelas de baixas velocidades relativa ao Sol, quentes, jovens (as mais brilhantes com $\sim 10^{7}-10^{8}$ anos), metálicas e localizadas principalmente nos braços galácticos. Com magnitudes absolutas variando entre $-8 \mathrm{e}+3$, as estrelas dessa população são principalmente do tipo O, B, cefeidas tipo I, objetos em aglomerados abertos e associações de estrelas. Já a População II foi caracterizada como estrelas mais velhas 
$\left(\sim 10^{10}\right.$ anos $)$ e frias, com altas velocidades e baixas metalicidades, situadas essencialmente no halo e no bojo da galáxia, sendo principalmente estrelas de aglomerados globulares, novas e nebulosas planetárias. As estrelas mais brilhantes da população II chegam a ser até 100 vezes mais fracas do que as estrelas mais brihantes da população I.

As populações estelares de Baade (1944) foram definidas basicamente através do diagrama cor-magnitude, numa época em que muitas fases evolutivas eram mal conhecidas. Uma vez que é sabido que o diagrama cor-magnitude de um conjunto de estrelas que se formaram ao mesmo tempo reflete o estado físico da evolução dessas estrelas, tais como suas massas, idade e composição química, a divisão das estrelas de uma galáxia em populações tem um sentido físico intrínseco. Historicamente, Baade (1944) conjecturou que galáxias elípticas seriam constituídas puramente por estrelas de população II, enquanto que galáxias espirais do tipo Sa e Sb apresentariam ambas as populações estelares, com as estrelas de população I restritas a região dos braços.

Hoje sabemos que as populações estelares não são binárias, i.e, existe um contínuo de populações, mas o conceito introduzido por Baade (1944) é ainda útil na caracterização das propriedades das estrelas de uma galáxia.

As populações estelares possuem propriedades muito importantes que nos ajudam a entender sua formação e evolução e, consequentemente, a compreender seu papel nas galáxias. Tais populações carregam diversas propriedades físicas notáveis, dentre as quais podemos destacar principalmente sua idade, metalicidade, cor, taxas e históricos de formação estelar e distribuição espacial, sendo essa última particularidade um dos focos do presente estudo.

\subsection{Síntese de Populações Estelares}

Para obtermos informações a respeito da história da formação estelar de uma galáxia, utilizamos frequentemente como ferramenta o estudo de seu espectro integrado.

Em geral, um espectro é composto pelo contínuo, linhas de absorção e linhas de emissão. O contínuo do espectro de uma galáxia normal é produzido por suas estrelas; as linhas de absorção são produzidas pela absorção da luz das estrelas em suas atmosferas, ou, ainda, absorvidas pelo gás e poeira; já as linhas de emissão surgem de regiões ionizadas por estrelas jovens (regiões HII), choques no meio interestelar ou por núcleos ativos de galáxias. A figura 1.1, apresenta dois espectros de galáxias, uma espiral e outra elíptica, obtidos do 
SDSS, além da identificação de algumas das principais linhas de emissão e absorção.

A luz integrada das galáxias é produzida pela mistura de todas as suas populações estelares (SP, do inglês Stellar Population), com idades e metalicidades que evoluem com o tempo e, a partir da decomposição do espectro nessas diversas populações, podemos compreender melhor as propriedades físicas da galáxia.

Apesar de ser um modo eficiente de estudar e entender as galáxias, o processo de recuperação de seu conteúdo através da decomposição do seu espectro é uma tarefa muito árdua. Para contornar esse problema, muitos métodos de síntese espectral têm sido desenvolvidos, os quais podemos separar em duas principais categorias: síntese empírica e em síntese evolutiva.

Trabalhos pioneiros de Beatrice Tinsley (Tinslev e Gunn, 1976; Tinsley, 1980), Hyron Spinrad e Benjamim Taylor (Spinrad e Taylor, 1972) estabeleceram as bases da síntese evolutiva levando em conta a evolução de um sistema estelar. Um conceito fundamental é o de Populações Estelares Simples (SSP, do inglês Simple Stellar Population), um conjunto de estrelas que se formaram ao mesmo tempo e com a mesma metalicidade. A população estelar de uma galáxia pode então ser considerada como a superposição de SSP's formadas ao longo da vida de uma galáxia. Para recuperar as informações a respeito das populações estelares, a síntese evolutiva faz uso de várias expressões analíticas para a função de massa inicial (IMF, do inglês Initial Mass Function), taxa de formação estelar e de bibliotecas com trajetórias evolutivas de estrelas. A partir dessas informações, calcula-se a evolução estelar das SSP's e se obtêm o espectro da galáxia em um tempo t, como sendo uma superposição do espectro dessas SSP's. Os trabalhos Bruzual e Charlot (2003) e Leitherer et al. (1999) são dois trabalhos clássicos de síntese de populações estelares via síntese evolutiva.

A técnica de síntese de populações empírica foi desenvolvida principalmente devido aos trabalhos de Morgan (1956), Wood (1966) e Faber (1972). Nesse método, um conjunto de características observáveis de uma dada galáxia é ajustada como sendo uma combinação linear de sistemas simples, tais como estrelas individuais ou SSP's, calculadas a partir da síntese evolutiva. O código de síntese espectral STARLIGHT (Cid Fernandes et al., 2005), descrito a seguir, é um exemplo de síntese de populações estelares que utiliza o método empírico. 
$\mathrm{RA}=27.87467, \mathrm{DEC}=13.13170, \mathrm{MJD}=51877$, Plate $=430$, Fiber $=256$

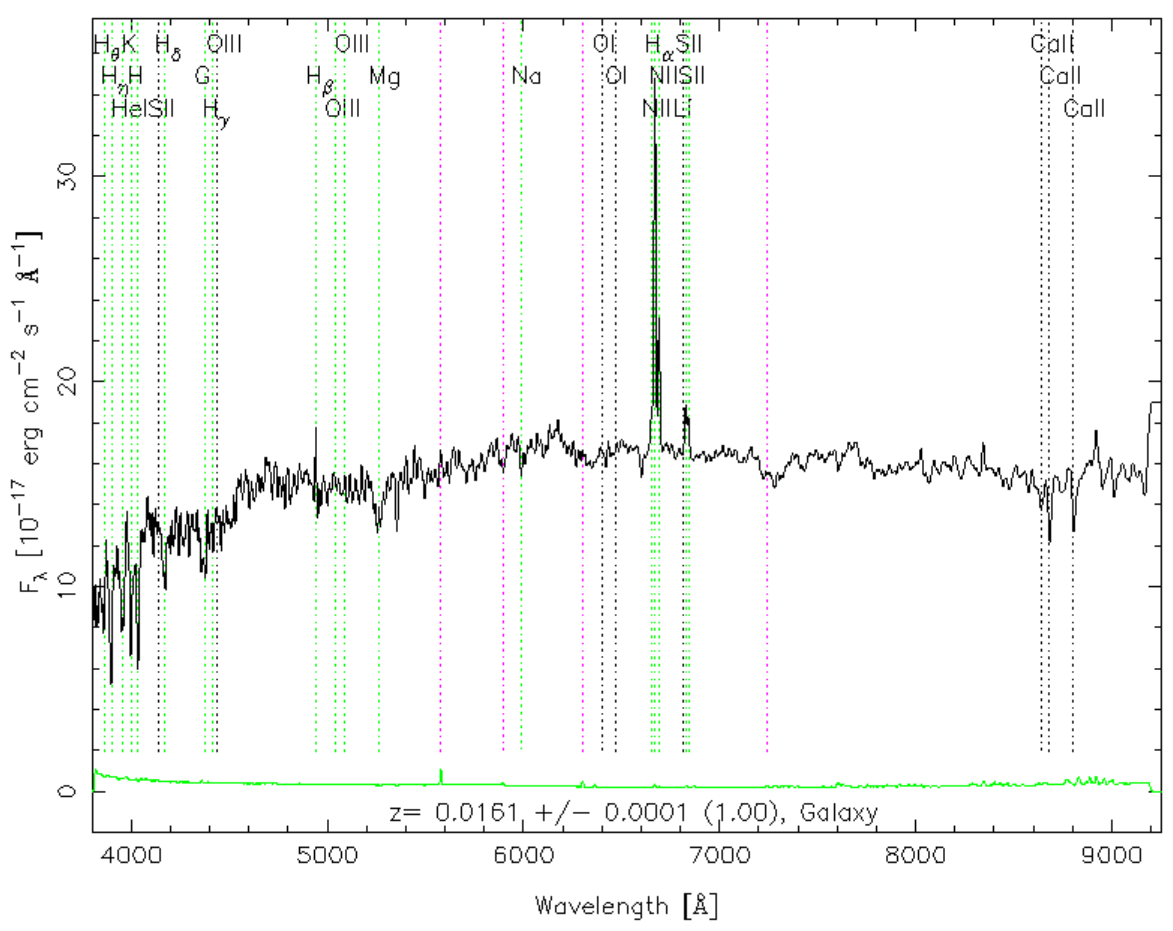

$\mathrm{RA}=181.11277, \mathrm{DEC}=1.89600, \mathrm{MJD}=52017$, Plate $=516$, Fiber $=36$

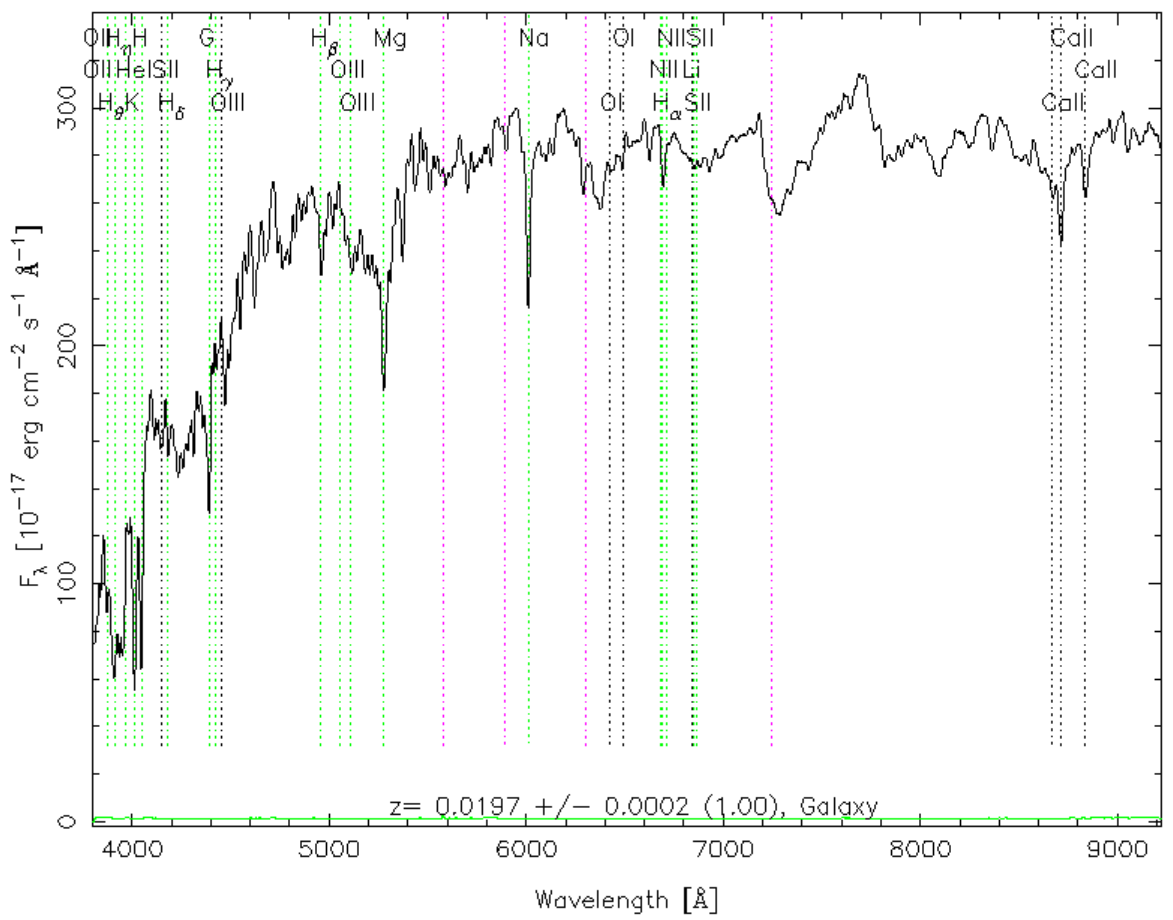

Figura 1.1: Espectros de uma galáxia espiral (acima) e outra elíptica (abaixo). Principais linhas características de absorção: Ca H+K (3934 $\AA, 3969 \AA)$, quebra em $4000 \AA, H \delta(4102 \AA)$,

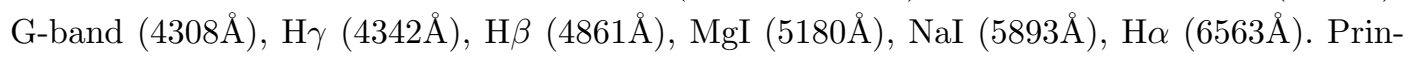

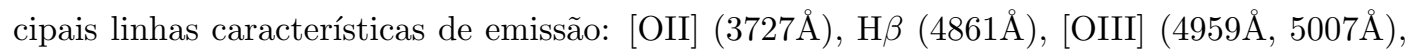
$\mathrm{H} \alpha(6563 \AA),[\mathrm{NII}](6548 \AA, 6584 \AA),[\mathrm{SII}](6716 \AA, 6731 \AA)$ 


\subsubsection{STARLIGHT}

O StARLight 1 (Cid Fernandes et al., 2005) é um código de síntese espectral que fornece informações a respeito das populações estelares das galáxias, tais como históricos de formação estelar e enriquecimento químico, extinções e dispersão de velocidade central. Ele emprega a técnica de síntese espectral semi-empírica, onde os espectros observados das galáxias são ajustados a partir de uma combinação de $N_{\star}$ SSP's.

Em Cid Fernandes et al. (2005), o código utiliza uma base de $N_{\star}=150$ SSP's obtidas de Bruzual e Charlot (2003), a biblioteca STELIB (Le Borgne et al., 2003), trajetórias evolutivas de Padova 1994 (Alongi et al., 1993; Girardi et al., 1996) e uma IMF dada por Chabrier (2003). As populações estelares simples são compostas por 25 idades $\left(10^{6} \leq\right.$ $t \leq 18 \times 10^{9}$ anos), 6 metalicidades $(0.0001 \leq Z \leq 0.05)$ e extinção estelar entre -1.0 e 4.0. Neste trabalho, serão utilizadas idades e metalicidades médias fornecidas pelo STARLIGHT, que são definidas como

$$
\begin{aligned}
\langle\log (t)\rangle_{L} & =\sum_{i=1}^{N_{\star}} \mathbf{x}_{\mathbf{i}} \log \left(t_{i}\right) \\
\langle Z\rangle_{L} & =\sum_{i=1}^{N_{\star}} \mathbf{x}_{\mathbf{i}} Z_{i}
\end{aligned}
$$

que são ponderadas pelo vetor de luz $\{\mathbf{x}\}$ das populaç oes da base.

Os estudos do STARLIGHT levaram a vários resultados interessantes como, por exemplo, sobre o modo como a bimodalidade observada nas galáxias pode estar diretamente ligada a idade média estelar ponderada pela luz (Mateus et al., 2006); a evolução de galáxias em ambientes densos é conduzida principalmente pela ação ambiental (Mateus et al., 2007); a evolução da relação massa-metalicidade (M-Z), em galáxias com massas menores do que $10^{10} M \odot$ são conduzidas principalmente pela história de formação estelar (Vale Asari et al., 2009), dentre outros resultados.

\subsection{Distribuição das Populações Estelares e suas Propriedades}

Os estudos realizados com o uso de espectros integrados têm acrescentado significativas informações a respeito da formação e evolução das galáxias no universo. Em particular, di-

1 http://www.starlight.ufsc.br/ 
versos trabalhos mais recentes têm investido esforços para estudar as propriedades das populações estelares espacialmente resolvidas dentro das galáxias. Estudar a distribuição espacial das populações estelares e suas propriedades é um modo viável de obter uma melhor compreensão sobre os processos e eventos experimentados pelas galáxias (Gargiulo et al., 2012). Estudar as variações espaciais das propriedades das populações estelares (idade, metalicidade, formação estelar, e até mesmo a presença de poeira) fornece, ainda, informações valiosas a cerca do cenário pelo qual as galáxias se formam.

No universo próximo, é bem estabelecido que as galáxias podem ser agrupadas em duas classes básicas: aquelas com morfologia do tipo disco, repletas de gás e sofrendo formação estelar (galáxias late-types); e aquelas que são dominadas por bojos, com pouco gás e cuja formação estelar cessou inteiramente (galáxias early-types) (Strateva et al., 2001; Kauffmann et al., 2003; Baldry et al., 2004; Mateus et al., 2006). É plausível postular que a bimodalidade da morfologia e das propriedades das galáxias esteja ligada a habilidade das mesmas em processar e transformar a massa de gás em estrelas, ou ainda em perdê-lo, tanto o gás contido em seu interior quanto nas regiões próximas a galáxia. Estudar as populações estelares resolvidas dessas galáxias tende a ajudar a entender o modo como o gás pode ser acretado e processado pela galáxia, de modo a reabastecer ou não seu conteúdo estelar.

Galáxias do tipo early-type são explicadas através de 3 principais cenários de formação: cenário monolítico, cenário hierárquico e cenário "inside-out". No cenário monolítico revisado, as galáxias early-types se formam pelo colapso de uma nuvem de gás que forma estrelas. A evolução dessas estruturas se dá então através do envelhecimento do seu conteúdo estelar (Larson e Tinsley, 1974; Katz, 1991; Arimoto, 1996; Kobayashi, 2004; Merlin e Chiosi, 2006). Neste cenário, devido ao poço de potencial, o gás rico em metais tende a fluir para o centro da galáxia, gerando uma população estelar mais rica em metais na região central do que nas regiões externas (gradiente radial de metalicidade negativo).

Dentro do cenário hierárquico, as galáxias early-types se formaram, em altos redshifts, através da fusão tanto de galáxias do tipo disco ricas em gás (fusão molhada, do inglês wet merger, Toomre e Toomre, 1972), quanto de galáxias quiescentes (fusão seca, do inglês dry merger, Bell et al., 2004). Na fusão molhada, a maior parte da massa estelar é formada através de surtos de formação estelar intensos (10-1000M $M_{\odot} /$ ano, Calzetti 2012) no centro da galáxias (Renzini, 2006), enquanto que na fusão seca o conteúdo estelar é basicamente a 
mistura do conteúdo estelar pré-existente nas galáxias que se fundiram. Nessas situações, o perfil radial de idade e metalicidade do conteúdo estelar tenderia a ser bastante significativo (com estrelas mais novas e metálicas no centro) no caso de fusão molhada e plano no caso de fusão seca, onde há a diluição das variações radiais de idade e metalicidade (Di Matteo et al., 2009).

Por último, o cenário mais recente de formação "inside-out", propõe que as galáxias se formam de dentro para fora (Daddi et al., 2005). Nesse contexto, as early-types são formadas como esferóides compactos ricos em gás. Em redshifts menores, as early-types sofrem fusões secas que vão adicionando camadas de baixa massa aos núcleos compactos, de modo que as regiões externas são mais novas que as internas (Hopkins et al., 2009; Wuyts et al.,,2010). Nesse cenário, as fusões secas não misturam o conteúdo estelar, mas rearranjam as populações estelares dos satélites acretados nas regiões periféricas das galáxias.

Galáxias do tipo espiral late-type são objetos que possuem uma componente do tipo disco e uma componente tipo bojo, onde a primeira se sobressai sobre a última. O paradigma clássico assume que tais objetos sejam formados através do colapso dissipativo de uma nuvem de gás em rotação (Fall e Efstathiou, 1980). Nesse cenário, as galáxias late-type possuem perfil de brilho exponencial na direção radial, além de um perfil de idade radial aproximadamente constante ou decrescente com o raio galáctico (Lin e Pringle, 1987; Ferguson e Clarke, 2001; Naab e Ostriker, 2006).

Num primeiro momento, pode-se conjecturar que as galáxias do tipo disco possuem formação estelar contínua ao longo do seu disco. Entretanto, Huang et al. (2013) mostrou que há uma fração considerável de galáxias late-types de baixa massa estelar e baixa densidade superficial que tem experimentado surtos recentes de formação estelar, com taxas maiores do que a habitual de $1-5 M_{\odot} /$ ano (Calzetti, 2012), tanto nas regiões externas quanto nas regiões internas. Neste mesmo trabalho, inclusive, Huang et al. (2013) afirmam que cerca de um terço das galáxias massivas $\left(\log M_{\star}>10.8\right)$ dominadas pela componente bojo experimentaram esses surtos de formação estelar recentes, mas apenas nas regiões externas $\left(R>0.7 R_{90}\right)$, pois a formação estelar nas regiões internas é suprimida em galáxias com razão bojo-disco grandes.

Bell et al. (2004) estudaram a correlação entre as idades e metalicidades com a posição das populações estelares em uma amostra de galáxias espirais, mostrando que o gradiente de idade nessas galáxias era maior do que o predito por modelos. Muñoz-Mateos et al. (2007), 
estudando o perfil radial da taxa de formação estelar específica de espirais, mostrou que as galáxias formam estrelas com taxas relativamente mais altas nas regiões externas, quando comparadas com as regiões internas. Wang et al. (2011) evidenciou que o gradiente de cor UV/óptico das galáxias são fortemente correlacionados com seu conteúdo de gás atômico. Galáxias ricas em gás com altas massas de gás HI tem gradiente de cor mais forte do que galáxias de mesma massa estelar, tamanho e conteúdo de gás HI médio. Além disso, o gradiente de cor UV/óptico das galáxias tem uma dependência muito forte tanto com a extinção dada pela poeira quanto pela formação estelar que ocorreu há menos do que $10^{9}$ anos (Kong et al., 2004).

Tanto Bell et al. (2004), quanto Muñoz-Mateos et al. (2007) e Wang et al. (2011) interpretam seus resultados como evidências do cenário de formação "inside-out".

As galáxias irregulares, por sua vez, são sistemas com estrelas velhas, cuja luz é dominadas pelas estrelas jovens e regiões HII. O que difere este tipo de objeto das demais galáxias late-type, basicamente, é o fato de não existirem braços espirais e a simetria imposta por tais subestruturas no disco das galáxias (Hunter, 1997), resultando numa aparência irregular. Entretanto, é justamente essa característica, a ausência de estrutura espiral, que faz desse tipo de galáxia um bom laboratório para o estudo das populações estelares e sua formação. Nesses objetos, pode-se investigar quais os mecanismos que regulam e distribuem o conteúdo estelar, uma vez que tais atividades não são conduzidas pela presença de braços.

As galáxias irregulares do tipo Sm são galáxias cuja estrutura espiral é fracamente identificada, enquanto que as do tipo Im são definidas pela ausência de estruturas organizadas. Ainda, as galáxias Im diferem das galáxias espirais também em termos das suas propriedades globais. As galáxias irregulares costumam ser mais azuis, menores, menos luminosas, menos massivas, mais ricas em gás, baixa metalicidade e menor quantidade de poeira. Uma vez que as irregulares possuem baixa metalicidade e grandes quantidades de gás, elas são entendidas como objetos menos desenvolvidos que as galáxias espirais, no sentido de que pouco do seu conteúdo interestelar foi convertido e processado pelas estrelas.

Dentre as galáxias irregulares, as Nuvens de Magalhães são duas galáxias muito próximas à nossa Galáxia e que, devido a isso, têm sido bastante estudadas (Dottori et al., 1996; van der Marel, 2001; van der Marel e Cioni, 2001). Maragoudaki et al. (1998, 2001) estu- 
daram a distribuição espacial das populações estelares em ambas as Nuvens de Magalhães, encontrando que a regularidade e ordenação da distribuição espacial do seu conteúdo estelar se correlaciona com o aumento de sua idade. As estrelas mais jovens encontradas em tais galáxias são creditadas como resultado de surtos de formação estelar, embora não haja ainda um consenso sobre os possíveis processos que desencadearam tais surtos (Belcheva et al., 2011).

Cioni et al. (2000) utilizou observações das Nuvens de Magalhães no infravermelho para distinguir três grupos de objetos com diferentes idades médias. Estudando a distribuição espacial desses três grupos, Cioni et al. (2000) observou que eles possuíam comportamentos distintos: enquanto as estrelas mais jovens se distribuem irregularmente sobre as $\mathrm{Nu}-$ vens de Magalhães, as estrelas mais velhas possuem uma distribuição suavemente regular. Com dados do 2MASS, Nikolaev e Weinberg (2000) e Gonidakis et al. (2009) analisaram o conteúdo estelar da Pequena Nuvem e Grande Nuvem, através de uma análise morfológica, idetificando diferentes populações e estimando sua distribuição projetada, encontrando que as populações estelares possuem um perfil exponencial, onde coordenadas centrais das populações coincidem entre si, mas diferem das coordenadas centrais obtidas da distribuição espacial do gás. Possivelmente, as principais características observadas sejam consequência da interação entre as Nuvens de Magalhães e a nossa Galáxia.

Novas e promissoras perspectivas de estudar as populações estelares nas Nuvens de Magalhães serão alcançadas com os dados que serão ofertados pelo projeto GAIA (Spite, 2002; Kučinskas et al., 2002b,a). Com as observações dessa missão, será possível resolver as estrelas dessas galáxias, possibilitando um estudo aprofundado das suas populações estelares e suas propriedades.

\subsubsection{Resultados do CALIFA}

O projeto CALIFA, cujos detalhes técnicos serão dados na seção 1.4.3, é um levantamento que fará espectroscopia tipo IFU (do inglês Integral Field Unit) de cerca de 600 galáxias. Ele fornecerá informações espacialmente resolvidas para estudar os seguintes tópicos:

- Distribuição de massa nas galáxias.

- Populações estelares: história de formação estelar em galáxias early-type e disco; 
relações entre as populações estelares e o meio ambiente.

- Propriedades do gás ionizado: traçadores da distribuição do gás ionizado e estimativas das abundâncias químicas do gás.

- Presença de núcleos ativos (AGN's).

- Medidas de propriedades cinemáticas: do gás, das populações estelares e das subestruturas.

Os dados divulgados no primeiro release do CALIFA (Data Release 1, Husemann et al., 2013), têm trazido novas informações importantes a cerca das populações estelares e suas propriedades nas galáxias do universo local. Dentre os trabalhos realizados foi analisada a dependência do crescimento da massa estelar com a massa das galáxias. Aplicando o código de síntese espectral STARLIGHT aos espectros do tipo IFU de 105 galáxias para derivar as propriedades das populações estelares, mostrou-se que as galáxias massivas se desenvolvem numa perspectiva "de dentro para fora" (inside-out). Com evidências de downsizing, observou-se que tanto as regiões internas quanto as externas crescem mais rápido conforme a galáxia for mais massiva (Pérez et al., 2013; García Benito et al., 2013). Já as galáxias com massas inferiores ao limite de $\sim 7 \times 10^{10} M_{\odot}$ possuem formação estelar ativa em quase toda a sua extensão. Ainda, encontrou-se que o gradiente radial de metalicidade se correlaciona com a densidade de massa estelar, evidenciando que a densidade local influencia na evolução das galáxias (González Delgado et al., 2013).

Sánchez et al. (2013) estudou a relação massa-metalicidade (M-Z) tanto global quanto localmente para 150 galáxias do CALIFA. Com $\sim 3000$ regiões HII resolvidas individualmente, derivou-se a abundância de oxigênio e a taxa de formação estelar associada com cada região e as correlacionou com a massa estelar. Corroborando com um cenário onde o gás é reciclado dentro das galáxias (modelo de caixa fechada), este estudo constatou que a relação M-Z também é satisfeita localmente, mas mostrou também que não existe nenhuma relação secundária entre massa estelar e a taxa de formação estelar, nem mesmo com a taxa de formação estelar específica.

Em um projeto preliminar, Ruiz-Lara et al. (2013) analisou os perfis de brilho superficial, distribuição de idade e metalicidade estelar, além das abundâncias de gás de uma amostra piloto de 5 galáxias espirais. Apesar de verificar comportamentos distintos para 
as 5 galáxias, observou que, aparentemente, as distribuições de idade e metalicidade não diferem entre espirais normais e espirais barradas. Ainda que tal trabalho seja limitado, mostrou-se promissor no uso dos dados ofertados pelo CALIFA Survey.

\subsection{Os Grandes Surveys Modernos}

O estudo rigoroso da distribuição das galáxias e suas propriedades demanda surveys muito profundos. Até o inicio dos anos 1980, catálogos como o de Shapley-Ames (Shapley e Ames, 1932; Sandage e Tammann, 1980) e o Reference Catalogue of Bright Galaxies (de Vaucouleurs et al., 1964, 1976, 1995) eram pouco profundos e tratavam as galáxias de modo individual; já os catálogos como o de Aglomerados de Abell registravam aglomerados como um todo, entretanto continuavam não sendo suficientemente profundos.

Subsequentemente, houve um salto quantitativo na qualidade dos dados fornecidos por grandes surveys, que passaram a conter informações de redshifts e, consequentemente, ofereceram um panorama tridimensional do céu. Dentre esses surveys, podemos citar o CfA Redshift Survey (Huchra et al., 1983), o Las Campanas Redshift Survey (Trac et al., 2002; Marzke et al., 1999; Shectman et al.,, 1996) e o 2 degree Field Galaxy Redshift Survey (Colless e Boyle, 1998; Díaz et al., 2005). A seguir, estão descritos três grandes surveys: um que revolucionou o modo como vemos o universo e que ainda produz muita ciência (seção 1.4.1) e outros dois surveys que irão causar uma nova mudança de paradigma quanto a nossa concepção de como as estruturas se formam (seção 1.4.3), além da possibilidade de contribuir para desvelar o caráter da energia escura (seção 1.4.2).

\subsubsection{SDSS/DR7}

Seguindo a tendência de busca por mais e melhores dados, o Sloan Digital Sky Survey (SDSS) é um dos maiores e mais importantes surveys fotométricos e espectroscópicos já realizados. Este grande projeto foi o primeiro a usar detectores CCD ao invés de placas fotográficas para um levantamento em grande escala, o que assegurou qualidade, homogeneidade e precisão controlada dos dados obtidos. Ao longo de sua operação, o SDSS mapeou mais de um quarto do céu, fornecendo imagens multicoloridas e profundas de mais de 350 milhões de objetos distintos, além de criar um mapa de distribuição tridimensional de mais de 930.000 galáxias, mais de 120.000 quasares e 460.000 estrelas. Desde o Early 
Data Release até o Data Release 10 (também conhecido como SDSS III)(Aihara et al., 2011), o SDSS imageou mais de 8000 graus quadrados do céu em cinco bandas ópticas u, g, r, i, z e obteve espectros de galáxias e quasares em cerca de 7500 graus quadrados do céu no hemisfério norte e 750 graus quadrados do céu no hemisfério sul (Abazajian et al., 2009).

$\mathrm{Na}$ figura 1.2, apresentamos a projeção em coordenadas equatoriais da cobertura espectroscópica e fotométrica das regiões que o SDSS mapeou no DR7. Já na figura 1.3, exibimos um mapa da distribuição de galáxias do SDSS. As regiões cobertas pelo Sloan Digital Sky Survey até o DR7 foram escolhidas de tal forma a evitar a extinção galáctica produzida no plano de nossa Galáxia.
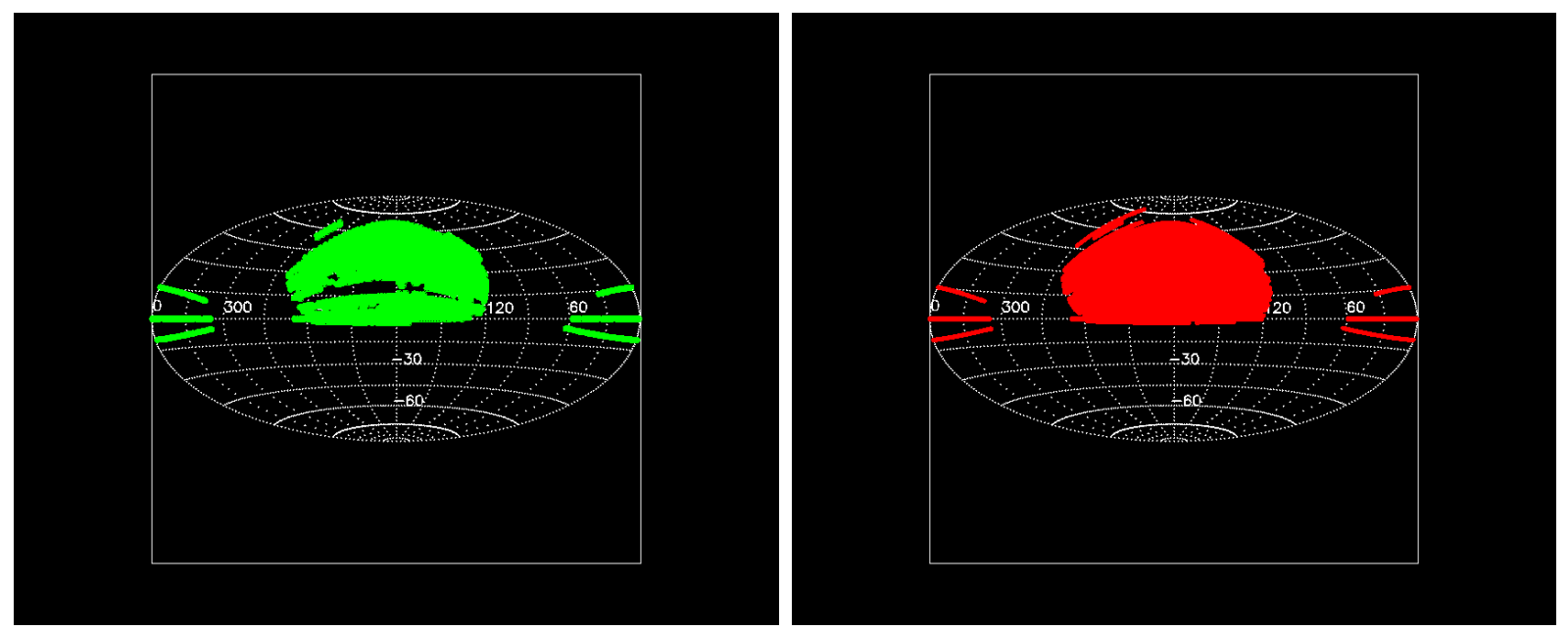

Figura 1.2: Levantamentos espectroscópico e fotométrico do DR7 do SDSS

\subsubsection{JPAS Survey}

Elaborado inicialmente com o objetivo de detectar Oscilações Acústicas de Bárions (BAO's), o projeto Javalambre Physics of the Accelerating Universe Astrophysical Survey (J-PAS) consiste de um levantamento a partir de um novo observatório astronômico e que irá mapear mais de 8000 graus quadrados do céu com 56 filtros. Este novo levantamento é uma colaboração entre astrônomos espanhóis e brasileiros.

Com uma combinação de dois telescópios com grande campo de visão, um de 2,5m para o levantamento e outro de $0,8 \mathrm{~m}$ para calibração, um conjunto de 14 CCDs de grande formato e um sistema de 54 filtros estreitos e 2 filtros largos, o J-PAS irá produzir imagens 


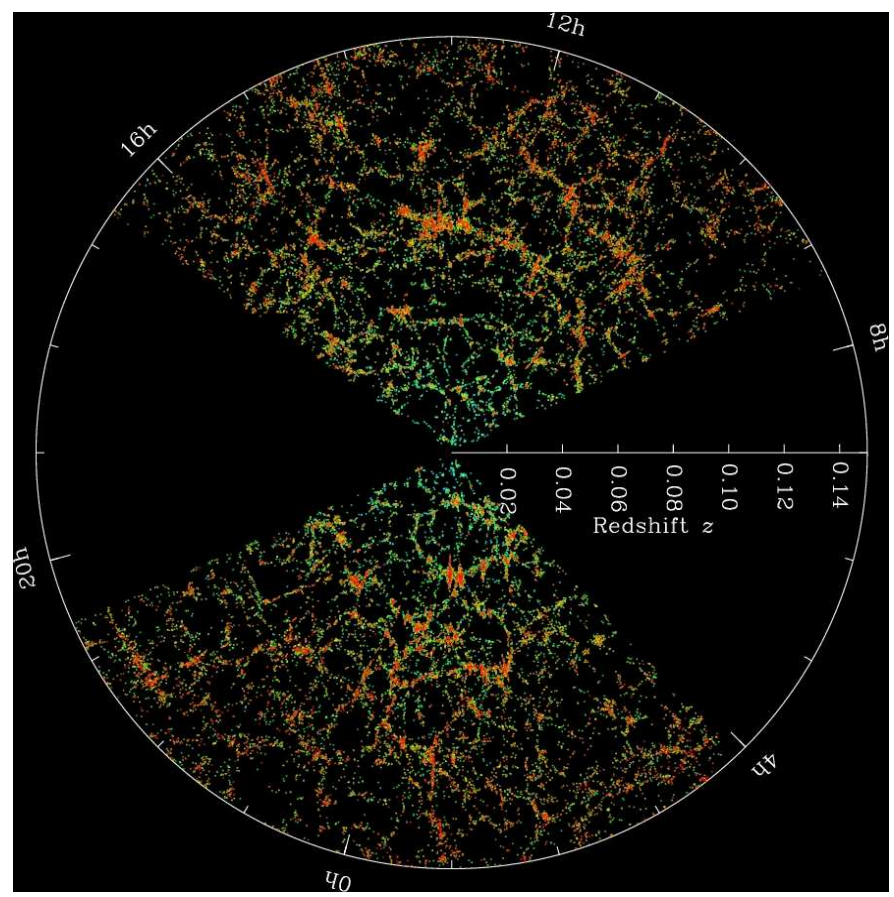

Figura 1.3: Mapa tridimensional da distribuição espectroscópica de galáxias do SDSS. Cada ponto representa uma galáxia com aproximadamente 100 bilhões de estrelas, e onde cada galáxia é colorida de acordo com a idade média de suas estrelas.

de alta qualidade e espectros de baixa resolução $(\mathrm{R} \sim 40)$ para cerca de $1 / 5$ do céu,

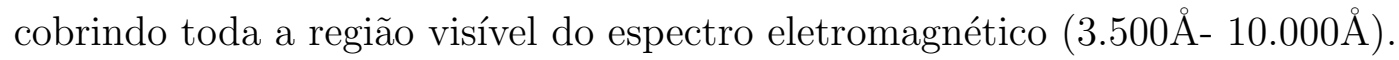

A articulação das imagens de alta qualidade e os espectros de baixa fornecerá dados e informações sem precedentes das dezenas de milhões de objetos que serão observados. Além da motivação inicial, o levantamento do J-PAS poderá ser usado em uma infinidade de aplicações, tais como o estudo da distribuição de energia escura no universo, a formação e evolução de galáxias, mapear a estrutura da nossa Galáxia, estudar quasares, supernovas e muito mais.

A previsão é que o mapeamento tenha início a partir de 2014 e que sua duração seja de 5 a 6 anos. A figura 1.4 mostra a cobertura espectral pretendida com os 56 filtros. 


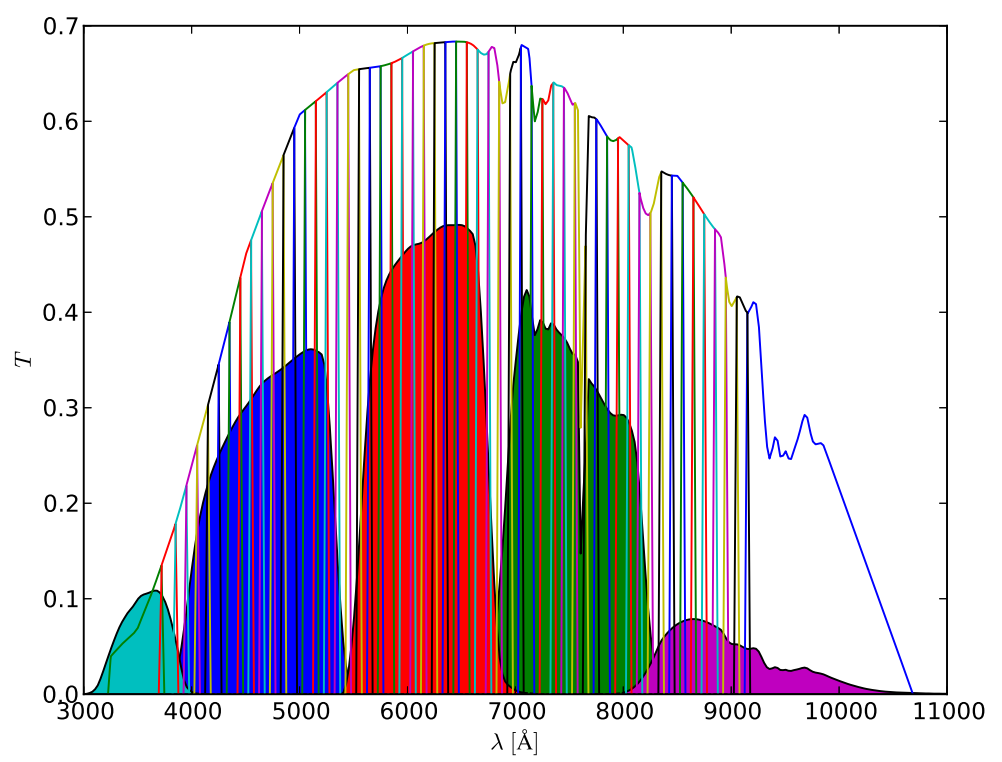

Figura 1.4: Definição teórica do conjunto de filtros do J-PAS. As regiões pintadas de azul, verde, vermelho, azul claro e magenta representam os filtros ugriz do SDSS e as curvas coloridas vazadas representam os 56 filtros do J-PAS, sendo o $1^{\circ}$ e o último filtros de banda larga. (Figura cedida gentilmente por William Schoenell.)

\subsubsection{CALIFA Survey}

O Calar Alto Legacy Integral Field Area Survey (CALIFA) é um grande projeto que está sendo realizado pelo Centro Astronômico Hispano-Germânico no observatório Calar Alto, onde serão obtidos espectros espacialmente resolvidos de 600 galáxias locais, por meio de espectroscopia de campo integral (IFS, do inglês integral field spectroscopy, Sánchez et al. 2012). As observações do CALIFA estão sendo realizadas com o espectrofotômetro Potsdam Multi Aperture Spectrograph (PMAS2, Roth et al. 2005), que está instalado no telescópio Calar Alto de 3,5 metros e com um campo de visão hexagonal grande, de 74 " $\times 64$ " (Verheijen et al., 2004; Kelz et al., 2006).

De uma amostra com 939 galáxias do DR7 do SDSS, foram observadas 600 galáxias, que foram selecionadas aleatoriamente, de acordo com as condições de observação. Utilizando a espectroscopia de campo integral, o survey realizado pelo CALIFA fornecerá uma grande e interessante quantidade de dados do tipo IFU de campo largo para as 600 galáxias. Com

\footnotetext{
${ }^{2}$ PMAS - Potsdam Multi Aperture Spectrograph, utilizado no modo PPAK, no qual as observações são feitas com um feixe de 331 fibras de ciência com um diâmetro de 2,7"/fibra cobrindo um hexágono de $72 " /$ de diâmetro.
} 
os dados fornecidos pelo CALIFA será possível aprimorar os estudos de AGN, formação estelar, fontes de ionização, gradientes de metalicidade e idade de populações estelares, dentre outras importantes áreas da astrofísica moderna.

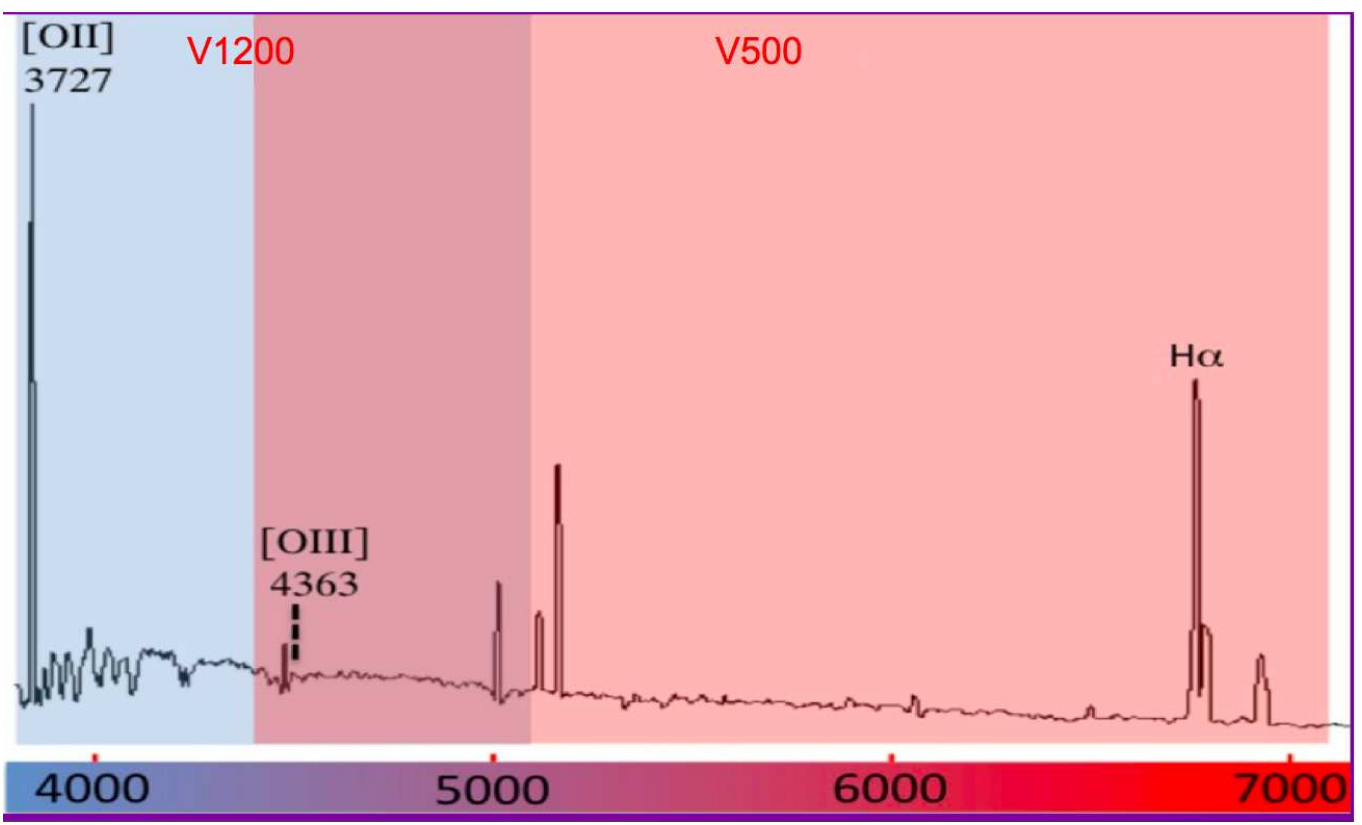

Figura 1.5: Cobertura espectral do CALIFA Survey: a região azul, vermelha e a região de sobreposição das duas bandas. Figura extraída de Marino et al. (2012)

As galáxias da amostra do CALIFA estão no universo local, com seus redshifts no intervalo $0,005<z<0,03$. A cobertura espectral se extende de $3500 \AA \sim 7000 \AA$, numa cobertura sobreposta (figura 1.5), sendo que as observações na faixa do vermelho ( $4300 \AA$ $7000 \AA$ ) foram realizadas com resolução espectral $R=850$, enquanto que as observações na faixa do azul $(3700 \AA \AA-5000 \AA)$ tiveram resolução $R \sim 1650$.

As observações realizadas pelo CALIFA tiveram início em meados de 2010. A figura 1.6 mostra o diagrama cor-magnitude com as galáxias do DR1, com um mapa do fluxo na banda V, mapa da idade das populações estelares, da velocidade do gás e da distribuição de $\mathrm{H} \alpha$ (zoom no canto esquerdo da imagem).

\subsection{Populações Estelares - Um outro approach}

Visto a importância do entendimento a respeito das populações estelares e, ainda, a relevância de entendê-las espacialmente dentro das galáxias, este trabalho se propõe a criar e testar ferramentas quantitativas para a análise das distribuições espaciais das populações 


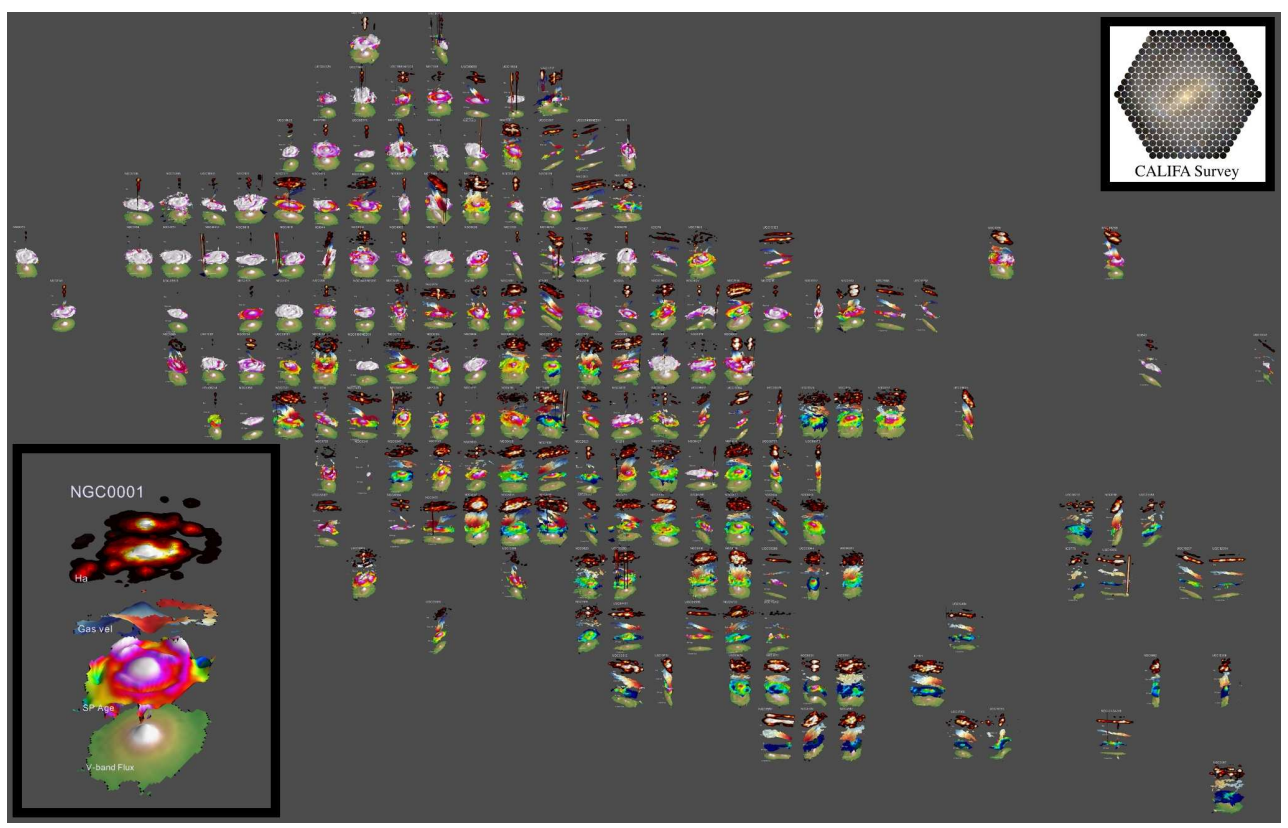

Figura 1.6: Diagrama cor-magnitude das galáxias já observadas pelo DR1 do CALIFA Survey. Figura obtida da página do 4th Busy Week do CALIFA, em 15 de julho de 2013.

estelares no interior das galáxias. As técnicas e ferramentas desenvolvidas neste trabalho são um projeto piloto voltado aos dados que serão ofertados em breve pelo projeto $J-P A S$, que, dado sua natureza, irá permitir análises espectrais píxel a píxel.

Para tanto, o presente trabalho traz uma análise fotométrica de uma pequena amostra de galáxias de diversos tipos, obtidas a partir do SDSS. Com o uso das magnitudes ugriz do SDSS, foi feita uma classificação espectral a partir de cores e foram adotados diversas ferramentas estatísticas para quantificar e explorar a distribuição espacial das populações estelares no interior das galáxias.

Esta dissertação se estrutura da seguinte forma: No capítulo 2 serão definidas as amostras utilizadas no estudo; no capítulo 3 será definido o conceito de populações estelares utilizado nesse estudo, além da definição e explicação do conceito de análise PCA; no capítulo 4 estão descritos os métodos e procedimentos utilizados no processamento dos dados; no capítulo 5 são apresentados os estimadores estatísticos que adotamos para as análises e, por último, no capítulo 6 estão apresentados os principais resultados obtidos no estudo, os quais são sumarizados no capítulo 7. 
Capítulo 2

\section{Base de dados}

Neste capítulo será feita a apresentação e descrição dos dados utilizados nesta pesquisa. A seção 2.1 caracteriza a amostra de 15 galáxias obtidas do projeto CALIFA, enquanto a seção 2.2 apresentará a amostra de dados do SDSS que foram utilizadas na análise PCA.

\subsection{Amostra de galáxias do CALIFA}

Uma amostra de galáxias de diferentes tipos foi selecionada a partir dos dados do First Data Release do projeto CALIFA (Sánchez et al., 2012), um empreendimento astronômico que pretende realizar espectroscopia de campo integral (IFU) de cerca de 600 galáxias. O objetivo de se escolher galáxias deste projeto é que pretendemos, mais tarde, refazer a análise apresentada nessa dissertação usando os espectros medidos píxel a píxel pelo CALIFA. As galáxias do CALIFA foram selecionados a partir do catálogo fotométrico do DR7/SDSS (Abazajian et al., 2009), de acordo com os seguintes critérios:

- $0,005<z<0,03$

- $45 " \leq D_{25} \leq 80 "$, na banda $r$ do SDSS;

O primeiro critério assegura que as galáxias estejam no universo local, enquanto que o critério quanto ao diâmetro isofotal $D_{25}$ garante que as galáxias sejam angularmente bem resolvidas e que tenha um bom ajuste com o campo de visão do espectrógrafo utilizado (PMAS). Nesta dissertação, estamos trabalhando com dados do DR7/SDSS para 15 galáxias desse levantamento, com tipos morfológicos variados, a fim de testarmos nossos métodos. Na tabela 2.1] são apresentadas algumas informações a respeito das 15 galáxias escolhidas, tais como os nomes dos objetos selecionados, suas coordenadas em ascensão reta 
e declinação, tipo morfológico obtido pelo NED ( The NASA/IPAC Extragalactic Database (Helou et al., 1995)), além dos redshifts e magnitudes de Petrosian nas bandas ugriz obtidos do DR7/SDSS. As imagens das galáxias foram agrupadas em elípticas e lenticulares E/S0 (figura 2.1), espirais SA/SB (figura 2.2) e espirais tardias (figura 2.3).

Tabela 2.1 - Nome, ascensão reta, declinação, tipo morfológico, redshift e magnitudes de Petrosian nas bandas ugriz das galáxias da amostra.

\begin{tabular}{|c|c|c|c|c|c|c|c|c|c|}
\hline Galáxia & RA(J2000) & $\operatorname{Dec}(J 2000)$ & Morfologia & Redshift & $\mathrm{u}$ & g & $\mathrm{r}$ & $\mathrm{i}$ & $\mathrm{z}$ \\
\hline NGC6173 & $16: 29: 44.87$ & $40: 48: 41.96$ & $\mathrm{E}$ & 0.029 & 15.28 & 13.22 & 12.36 & 11.95 & 11.78 \\
\hline NGC6125 & $16: 19: 11.53$ & $57: 59: 02.89$ & $\mathrm{E}$ & 0.015 & 14.93 & 12.99 & 12.16 & 11.73 & 11.51 \\
\hline NGC6146 & $16: 25: 10.32$ & $40: 53: 34.31$ & E? & 0.029 & 15.29 & 13.35 & 12.55 & 12.11 & 11.88 \\
\hline NGC1349 & $03: 31: 27.51$ & $04: 22: 51.24$ & So & 0.022 & 16.49 & 14.21 & 13.21 & 12.64 & 12.37 \\
\hline UGC05771 & $10: 37: 19.33$ & $43: 35: 15.31$ & $\mathrm{~S} 0 / \mathrm{a}$ & 0.025 & 16.02 & 14.14 & 13.26 & 12.83 & 12.57 \\
\hline NGC0776 & $01: 59: 54.52$ & $23: 38: 39.39$ & $\mathrm{SAB}(\mathrm{rs}) \mathrm{b}$ & 0.016 & 15.75 & 13.75 & 12.85 & 12.49 & 12.45 \\
\hline NGC2916 & $09: 34: 57.60$ & $21: 42: 18.94$ & $\mathrm{SA}(\mathrm{rs}) \mathrm{b} ?$ & 0.012 & 14.87 & 13.19 & 12.63 & 12.32 & 11.97 \\
\hline NGC7819 & $00: 04: 24.50$ & $31: 28: 19.22$ & $\mathrm{SB}(\mathrm{s}) \mathrm{b}$ & 0.016 & 16.06 & 14.28 & 13.62 & 13.48 & 13.68 \\
\hline UGC03253 & $05: 19: 41.88$ & 84:03:09.43 & $\mathrm{SB}(\mathrm{r}) \mathrm{b}$ & 0.013 & 15.68 & 13.81 & 13.14 & 12.77 & 12.66 \\
\hline NGC4185 & $12: 13: 22.19$ & $28: 30: 39.46$ & $\mathrm{Sbc}$ & 0.013 & 15.71 & 13.26 & 12.55 & 12.25 & 12.33 \\
\hline UGC00312 & $00: 31: 23.92$ & $08: 28: 00.23$ & $\mathrm{SB} ?$ & 0.014 & 15.39 & 14.00 & 13.59 & 13.45 & 13.12 \\
\hline UGC05358 & $09: 58: 47.13$ & $11: 23: 19.31$ & $\mathrm{SB}(\mathrm{s}) \mathrm{b}$ & 0.0097 & 16.63 & 15.34 & 14.77 & 14.67 & 15.04 \\
\hline UGC09665 & $15: 01: 32.46$ & $48: 19: 10.93$ & $\mathrm{Sc}$ & 0.009 & 15.99 & 14.23 & 13.53 & 13.13 & 12.92 \\
\hline UGC10331 & $16: 17: 21.12$ & $59: 19: 12.46$ & S pec & 0.015 & 15.75 & 14.43 & 13.99 & 13.78 & 13.87 \\
\hline NGC3991 & $11: 57: 30.95$ & $32: 20: 13.28$ & Im pec edge-on & 0.011 & 14.86 & 13.92 & 13.55 & 13.42 & 13.40 \\
\hline
\end{tabular}

\subsection{Amostra de galáxias do SDSS}

Muitos trabalhos têm mostrado que o uso de análises de componentes principais tem um grande potencial, uma vez que tal tipo de análise permite uma classificação espectral bastante acurada. Sendo assim, para mostrar a potencialidade deste método, foram selecionadas galáxias do DR7/SDSS (Abazajian et al., 2009) que atendessem aos seguintes critérios:

- Objetos com espectroscopia e cores;

- $0,005 \leq z \leq 0,03$;

- Raio de Petrosian na banda $r \geq 25$ "; 
- Flags na banda r para evitar objetos espúrios.

Utilizando a ferramenta CasJobs 1 fornecida pelo SDSS, foi obtida uma amostra com 1497 galáxias do DR7/SDSS. Ainda, mesmo com o uso das flags na banda r, foram utilizados diagramas cor-magnitude e cor-cor (figuras 2.4, 2.5) para determinar restrições nas cores, a fim de remover possíveis outliers. Essas restrições foram as seguintes:

- $0.7<u-g<2.3$

- $0.1<g-r<1.0$

- $-0.3<i-z<0.6$

- $r-i>0$

Assim sendo, 1396 galáxias foram selecionadas para a amostra final de galáxias do SDSS. No capítulo 3, iremos examinar estes dados no contexto da classificação espectral via análise PCA.
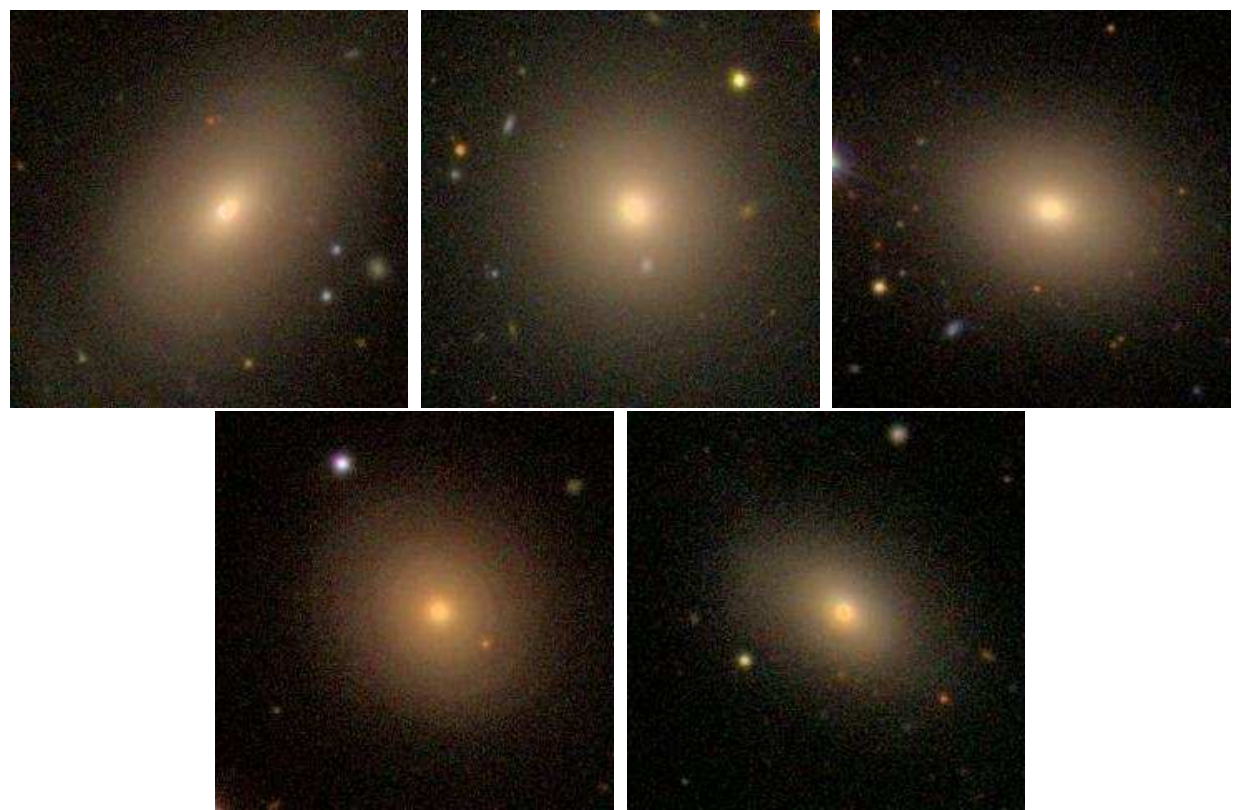

Figura 2.1: Galáxias elípticas e lenticulares da amostra. Imagens do SDSS/DR7.

\footnotetext{
${ }^{1}$ http://skyserver.sdss3.org/casjobs/
} 


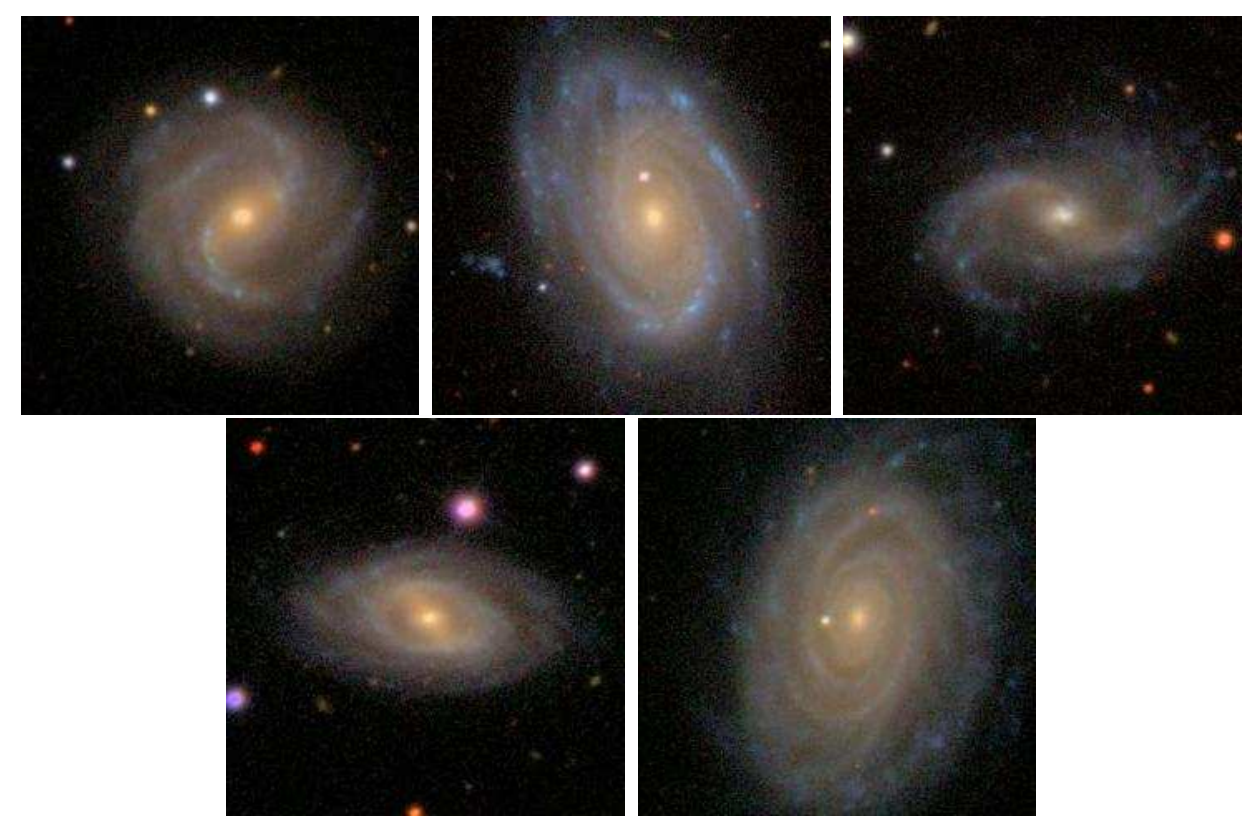

Figura 2.2: Galáxias espirais SA/SB da amostra. Imagens do SDSS/DR7.

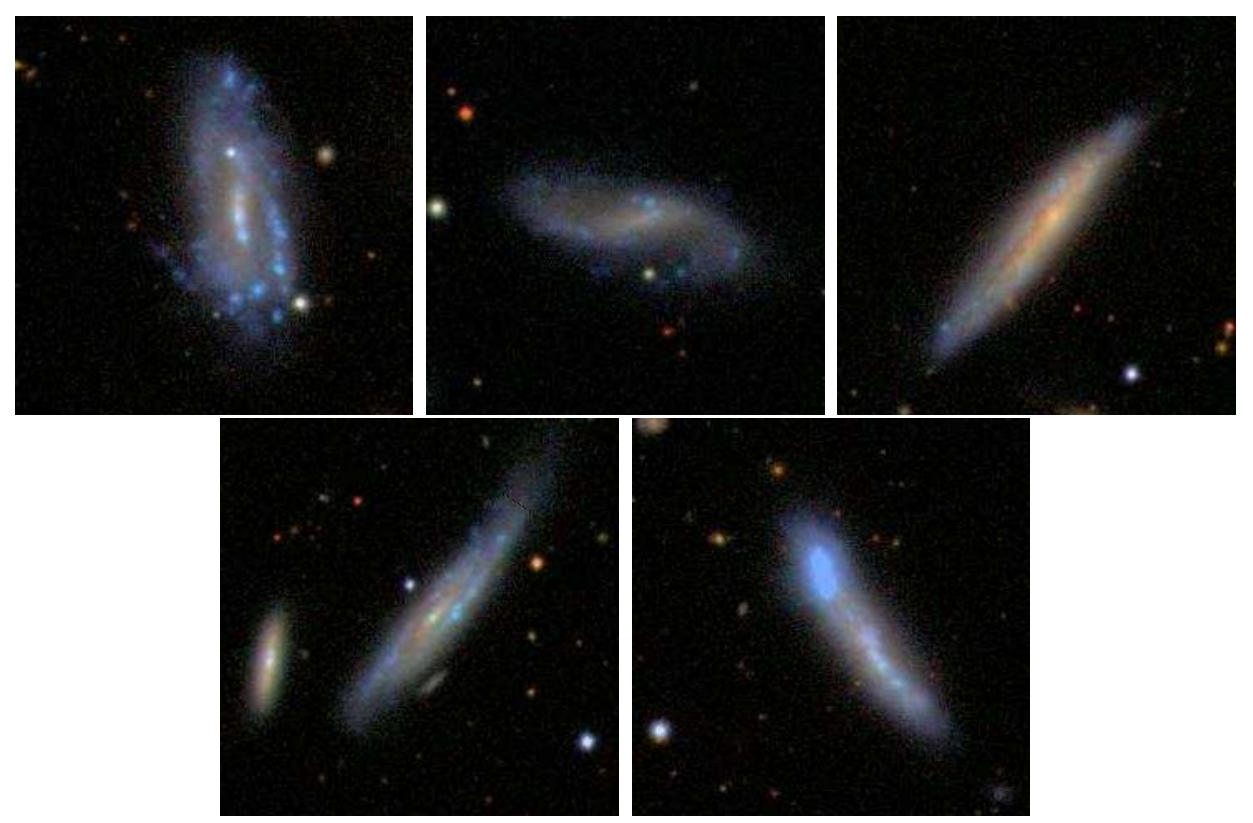

Figura 2.3: Galáxias espirais tardias da amostra, com os objetos irregulares e peculiares. Imagens do SDSS/DR7. 

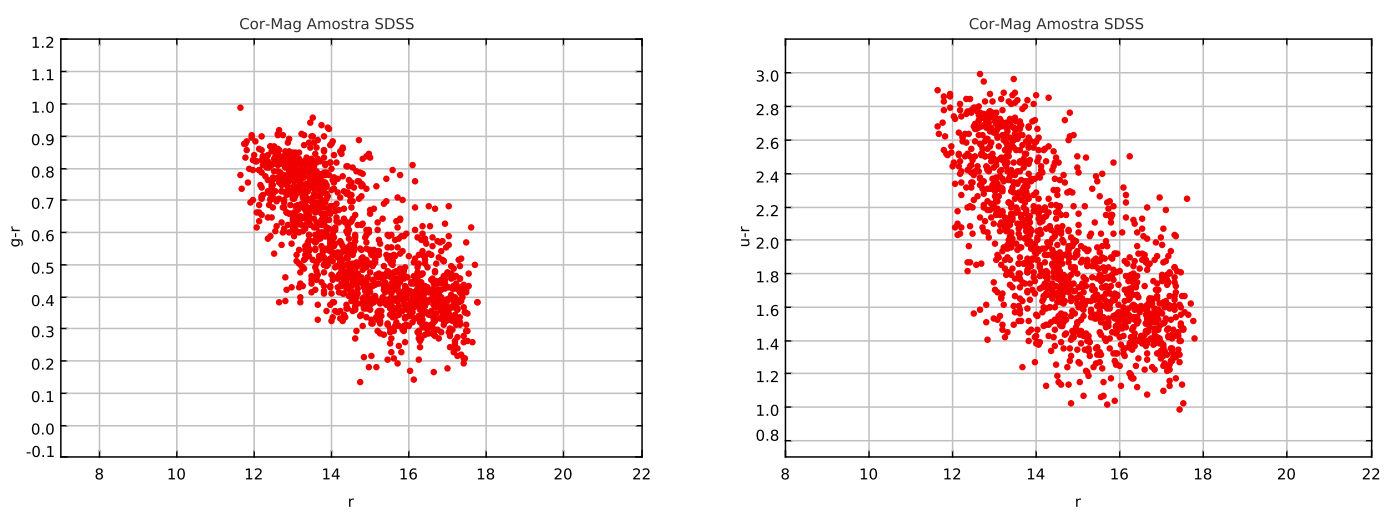

Figura 2.4: Diagramas cor-magnitude, $g-r$ versus r e $u-r$ versus r, para a amostra de galáxias obtidas a partir do DR7/SDSS.
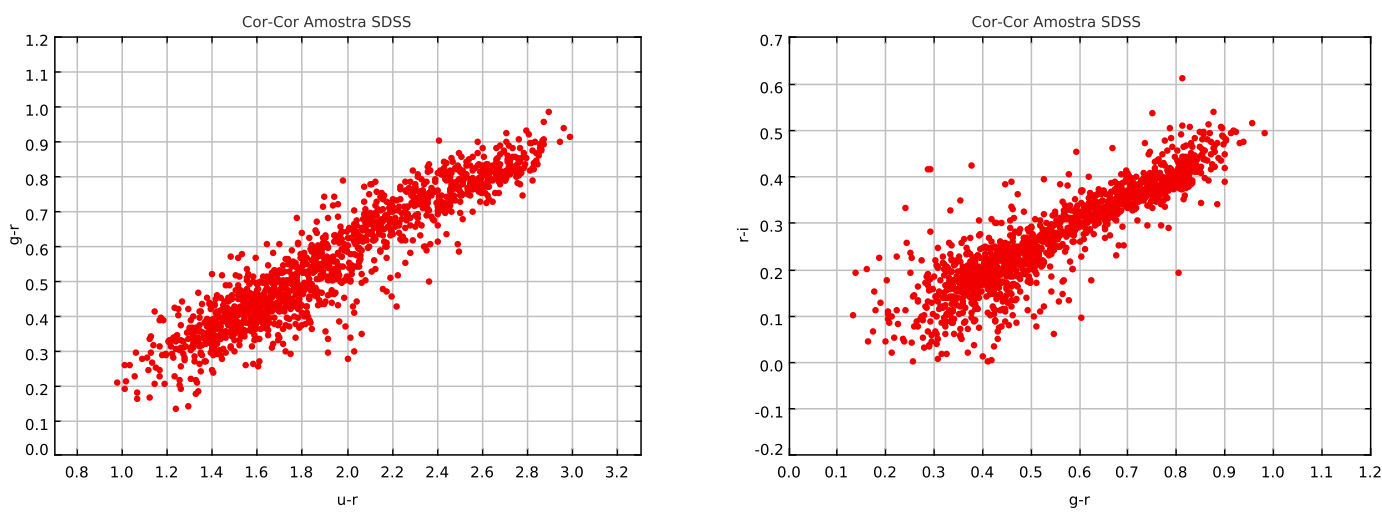

Figura 2.5: Diagramas cor-cor, $u-r$ versus $g-r$ e $g-r$ versus $r-i$, para a amostra de galáxias obtidas a partir do DR7/SDSS. 
Capítulo 3

\section{Populações Estelares}

Como visto na introdução desse trabalho, o estudo das populações estelares é elemento chave no entendimento de como se formam e evoluem as galáxias. Dado isso, neste capítulo será mostrado que as populações estelares de uma galáxia podem ser modeladas através da análise PCA das magnitudes ugriz das mesmas. Na seção 3.1 será feita uma explanação da análise PCA e seu potencial uso como proxy de populações estelares. Isto será demonstrado na seção 3.2. onde será feita a aplicação da análise PCA às galáxias do SDSS. Já na seção 3.3 será feita a descrição da aplicação de tal método nas galáxias da amostra escolhida para este trabalho.

\subsection{Análise de Componentes Principais - PCA}

Análise de Componentes Principais é uma poderosa ferramenta estatística com a qual podemos descrever um conjunto de observáveis possivelmente correlacionados através de uma base de vetores linearmente não correlacionados.

O uso de PCA é bastante difundido na astronomia, sendo aplicado em diversas áreas (Andreon et al., 2000; Zhao et al., 2009; Muñoz Bermejo et al., 2013; Lara-López et al., 2013; Carruba, 2013; Harnois-Déraps e Pen, 2013). Em particular, o uso da análise de componentes principais para determinação de tipos espectrais tem sido muito importante, principalmente pela sua aplicação aos dados de grandes levantamentos (Connollv et al., 1995; Folkes et al., 1996; Sodré e Cuevas, 1997; Sodré e Stasińska, 1999; Yip et al., 2004; McGurk et al., 2010).

Como descrito em Murtagh e Heck (1987), entre os principais objetivos da análise PCA, podemos citar os seguintes: 
- Redução de dimensionalidade;

- Determinação de uma combinação linear de variáveis;

- Seleção de características: a escolha de variáveis mais úteis na descrição do problema;

- Visualização de dados multidimensionais;

- Identificação de grupos de objetos ou de outliers.

Criado por Pearson (1901) e Hotelling (1933), a PCA utiliza um conjunto de dados representado por uma matriz $\mathrm{S}$ de $N$ registros por $M$ atributos, que podem estar correlacionados, e sumariza esse conjunto por eixos não correlacionados (componentes principais) que são uma combinação linear das $M$ variáveis originais. A análise via PCA é feita de forma que as componentes são ordenadas por sua variância.

Se há uma galáxia com $\mathrm{N}$ píxeis, é possível descrever cada píxel como um vetor $\boldsymbol{X}$ com $M$ magnitudes. Sendo $S$ um espaço $M$-dimensional gerado pelas $M$ magnitudes, tem-se que cada píxel da galáxia é um ponto no espaço $\boldsymbol{S}$

$$
\mathbf{S}=\left(\begin{array}{cccc}
S_{11} & S_{12} & \ldots & S_{1 M} \\
S_{21} & S_{22} & \ldots & S_{2 M} \\
\ldots & & & \\
S_{N 1} & S_{N 2} & \ldots & S_{N M}
\end{array}\right)
$$

onde $S_{N M}$ é a magnitude do $N$-ésimo píxel na banda $M$. Desse modo, todos os píxeis do objeto irão gerar uma nuvem de pontos nesse espaço.

Dado que as cores de uma galáxia estão correlacionadas com as populações estelares que a compõem, é de se esperar que os píxeis de uma galáxia se arranjem ao longo de uma linha, de uma direção preferencial dentro do espaço $\boldsymbol{S}$. Entretanto, dado que visualizar e analisar um espaço $M$-dimensional pode ser inviável, utilizar a análise PCA para reduzir a dimensionalidade do problema é uma alternativa muito eficiente de trabalhar tais dados.

Seja uma galáxia com seus píxeis representados por uma matriz $\mathbf{S}$ de $N$ píxeis em um espaço multidimensional de $M$ características $(\mathrm{M}=5$, magnitudes ou contagens nas bandas ugriz). Seja $S_{i j}$ o i-ésimo píxel na j-ésima banda. Estabelecendo o centróide dos pontos como a média $\bar{S}_{j}$ de cada atributo (eixo), podemos definir a variância em cada atributo (j-ésima banda) como sendo 


$$
\mathbf{V}_{\mathbf{j}}=\frac{1}{n-1} \sum_{i=1}^{m}\left(S_{i j}-\bar{S}_{j}\right)^{2} .
$$

O grau com que cada atributo $j$ é linearmente correlacionado com outro atributo $k$ é dado pela matriz de covariância

$$
\mathbf{C}_{\mathbf{j k}}=\frac{1}{n-1} \sum_{i=1}^{n}\left(S_{i j}-\bar{S}_{j}\right)\left(S_{i k}-\bar{S}_{k}\right) .
$$

Caso se deseje trabalhar com a matriz de correlação, é necessário normalizar cada variável de modo que a variância em cada variável seja igual a 1. Nesse caso, a matriz de correlação $r_{j k}$ é dada por

$$
\mathbf{r}_{\mathbf{j k}}=\frac{C_{j k}}{\sqrt{V_{j} V_{k}}} .
$$

Trabalhando com dados de modo que a média de cada eixo seja nula $\left(S_{i j}^{\prime}=S_{i j}-\bar{S}_{j}\right)$, podemos reescrever a matriz de covariância como sendo

$$
\mathbf{C}_{\mathbf{j k}}=\frac{1}{n-1} \sum_{i=1}^{n}\left(S_{i j}^{\prime} S_{i k}^{\prime}\right) .
$$

Seja $\boldsymbol{Y}$ um novo vetor, obtido pela transformação

$$
Y=\mathbf{A} X
$$

com A sendo a matrix $M \times M$ cujas linhas são os autovetores da matriz de covariância $\mathbf{C}$.

Pode-se mostrar que, nesse caso, a matriz de covariância do vetor $\boldsymbol{Y}$ é diagonal, com seus valores iguais aos autovalores da matriz $\boldsymbol{A}$, de modo que cada vetor $y_{k}$ que compõe $\boldsymbol{Y}$ é não correlacionado. Além disso, cada autovalor $\lambda_{\mathbf{k}}$ é igual a variância do k-ésimo elemento de $\boldsymbol{Y}$ :

$$
\lambda_{\mathrm{k}}=V_{k}
$$

Sendo $\boldsymbol{A}$ uma matriz real e simétrica, com transposta $A^{-1}=A^{\prime}$, podemos reverter o processo e, a partir da matriz $\boldsymbol{Y}$, reconstruir $\boldsymbol{X}$ :

$$
A^{-1}=A^{\prime} \rightarrow \mathbf{X}=A^{\prime} \mathbf{Y}
$$

Ainda, pode-se obter uma estimativa de $\boldsymbol{X}$ a partir dos $k$ maiores autovalores de $\boldsymbol{Y}$, com uma matriz $\mathbf{A}_{\mathbf{k}}$ contendo os $k$ autovetores correspondentes. Sendo $\boldsymbol{Y}$ agora kdimensional, a reconstrução de $\boldsymbol{X}$ será na verdade uma aproximação, obtida com $\mathbf{A}_{\mathbf{k}}$, sendo 
que a escolha dos autovetores com os $k$ maiores autovetores garante uma minimização no erro dessa aproximação. Assim, $\mathbf{X}$ ' será a aproximação obtida através da matriz $\mathbf{A}_{\mathbf{k}}$, dada por

$$
X^{\prime}=\mathbf{A}_{\mathbf{k}}^{\prime} Y
$$

Dado que a análise via PCA é uma ferramente efetiva na redução de dimensionalidade e na descrição de propriedades de um conjunto de dados, utilizaremos tal ferramenta para mostrar que é possível, a partir de cores e/ou magnitudes, atribuir um tipo espectral a uma galáxia através de suas componentes principais. A fim de se certificar que este tipo de análise é efetiva, aplicamos o método numa amostra de galáxias do DR7/SDSS.

\subsection{PCA do SDSS}

De modo análogo ao trabalho de Sodré e Cuevas (1997), que usaram o método de análise PCA para investigar propriedades estatísticas dos espectros integrados de galáxias, criou-se uma classificação espectral utilizando as cores ugriz do SDSS. A amostra de galáxias do SDSS foi selecionada como descrito no capítulo anterior e contém 1396 galáxias.

Tabela 3.1 - Componentes principais e respectivos autovalores e variâncias, em porcentagem e porcentagem acumulada.

\begin{tabular}{cccc}
\hline \hline Componente Principal & Autovalor & Porcentagem & Porcentagem Cumulativa \\
\hline 1 & 3.2356 & 80.8892 & 80.8892 \\
2 & 0.4438 & 11.0948 & 91.9839 \\
3 & 0.2103 & 5.2565 & 97.2404 \\
4 & 0.1104 & 2.7596 & 100.0000 \\
\hline
\end{tabular}

Empregando como base o método de análise proposto em Murtagh e Heck (1987), aplicamos a análise via PCA da matriz de correlação nas magnitudes das 1396 galáxias da amostra, de modo a descrever as informações através dos eixos principais. A tabela 3.1 exibe os autovalores e variância para as 4 primeiras componentes principais, tanto em porcentagem absoluta quanto em porcentagem acumulativa. Pode-se ver que a maior parte das variâncias estão concentradas nas duas primeiras componentes principais $(\sim 92 \%)$, 


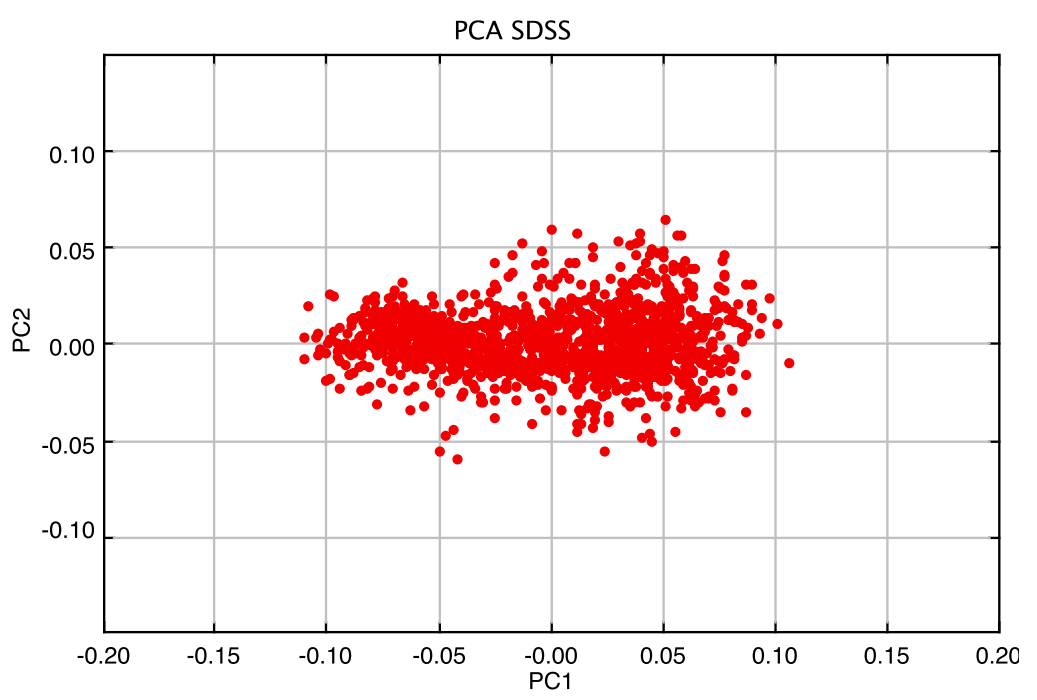

Figura 3.1: A segunda componente principal em função da primeira componente principal para a amostra de galáxias do SDSS.

mostrando que as informações fornecidas pelas 5 magnitudes de cada galáxia podem ser sumarizadas por essas duas componentes. A figura 3.1 apresenta a PC2 em função da PC1, sintetizando a dependência da primeira com a segunda componente principal, onde se observa que a variância da primeira componente é maior do que a segunda (por construção). Já na figura 3.2 estão representadas as cores $u-g, g-r, r-i, i-z$ das galáxias em função da primeira componente principal. É possível perceber que existe uma correlação muito boa entre as cores da galáxia e a primeira componente principal. É esta correlação que justifica o grande uso de PCA em classificação espectral.

Além das cores, as idades e metalicidades são outras duas propriedades físicas relacionadas a classificação espectral das galáxias. Para cada galáxia da nossa amostra de galáxias do SDSS, foram obtidas as idades e metalicidades, ambas ponderadas pelo fluxo, a partir dos dados obtidos pelo código de síntese espectral STARLIGHT, que, como visto na introdução, derivou diversas propriedades físicas das galáxias do SDSS/DR7. Assim, como complemento da demonstração da efetividade do uso da análise PCA na determinação de tipos espectrais de galáxias do SDSS, na figura 3.3 estão as correlações entre a primeira componente principal e as idades e metalicidades, ponderadas pelo fluxo, para as galáxias da amostra de galáxias do SDSS. Assim como no caso das cores, a primeira componente principal possui uma boa correlação tanto com a idade quanto com a metalicidade das 

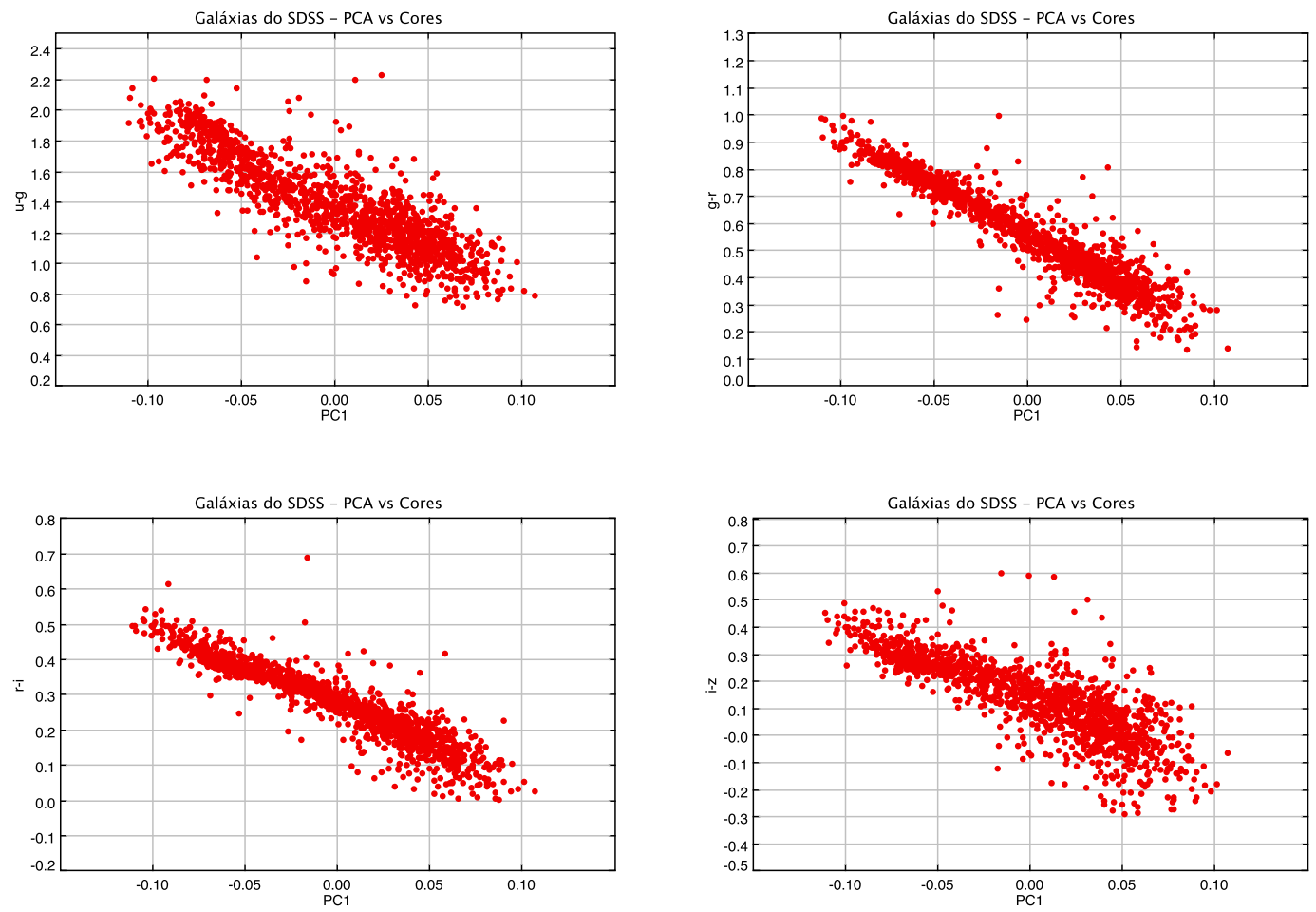

Figura 3.2: Galáxias do SDSS: As cores $u-g, g-r, r-i$ e $i-z$ em função da primeira componente principal.

galáxias, mostrando que a associação entre a primeira componente principal com um tipo espectral faz sentido.

\subsection{PCA das cores em cada píxel}

Uma vez que a análise PCA das galáxias se apresentou como uma alternativa válida no estudo de populações estelares, este trabalho propõe que tal recurso seja utilizado para caracterizar e classificar as populações estelares píxel a píxel de uma galáxia.

Para realizar a redução das informações fornecidas pelas magnitudes dos píxeis da galáxia em suas componentes principais, novamente foi empregado uma rotina fundamentada no código de análise PCA proposto por Murtagh e Heck (1987). Denominamos essa rotina como PCA_S3P. A partir de um arquivo de texto contendo as magnitudes em cada píxel, a rotina calcula as $\mathbf{M}=5$ magnitudes (ugriz) 1, as componentes principais e calcula

\footnotetext{
${ }^{1} \mathrm{Na}$ verdade, a análise foi feita com as contagens em cada píxel (com o céu subtraído). Como estamos usando a matriz de correlação, que centra e normaliza os dados, a PCA das magnitudes e contagens são muito parecidas.
} 

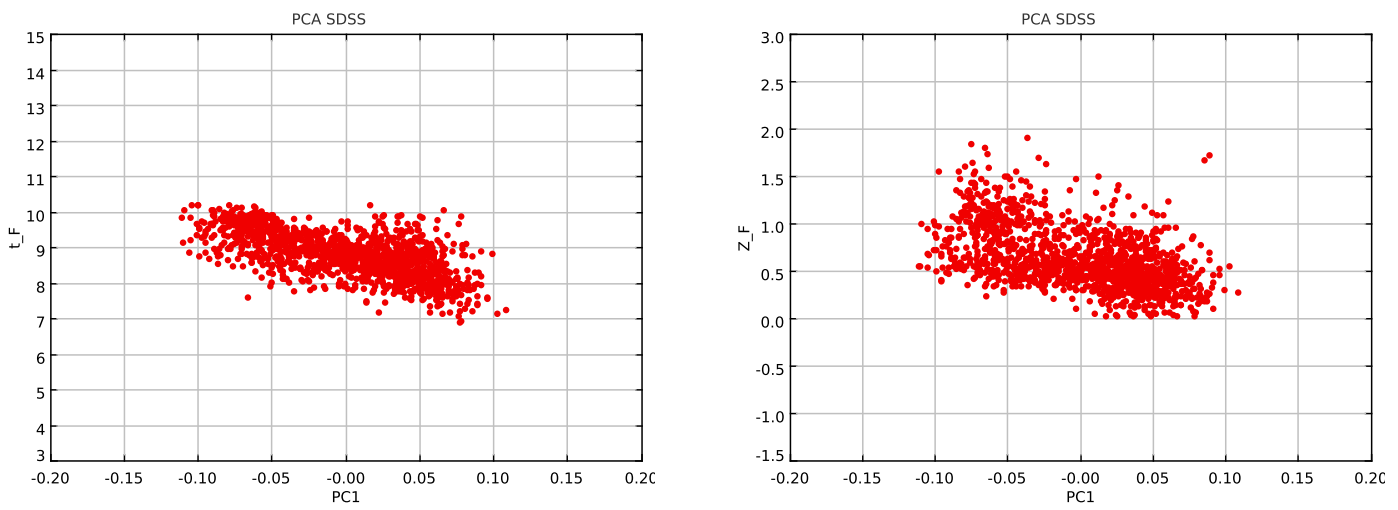

Figura 3.3: A primeira componente principal em função da idade $\log \left(t_{F}\right)$ (a esquerda) e em função da metalicidade $\log \left(Z_{F}\right)$ (a direita), ambas ponderadas pelo fluxo, para a amostra de galáxias do SDSS.

a projeção dessas cores nesses eixos. Dentre as opções possíveis de método de análise PCA oferecidas no código original, a rotina utiliza a análise PCA da matriz de correlação. Como output, a rotina retorna um arquivo contendo a matriz de correlação, os autovetores e seus autovalores associados e as projeções de cada píxel nos eixos principais calculados. A figura 3.4 mostra um exemplo de output obtido com a rotina PCA_S3P.

A título de exemplo, nas figuras 3.5 e 3.6 estão expostos a segunda componente principal e as cores $u-g, g-r, r-i$ e $i-z$ em função da primeira componente principal, respectivamente, para a galáxia espiral NGC2916, onde os valores médios da PC1 e das cores em cada população estão representados em azul. Novamente, como no caso da análise PCA nas galáxias da amostra do SDSS, é notável a correlação entre a PC1 e as cores em cada píxel, ainda que a dispersão dos dados seja bastante significativa no caso das cores $u-g$ e $i$ - $z$. 
CORRELATION MATRIX FOLLOWS.

\begin{tabular}{|c|c|c|c|c|c|}
\hline \multicolumn{6}{|c|}{1.0000} \\
\hline 0.4 & 1.00 & 000 & & & \\
\hline 0.4 & 0.79 & 1.0000 & & & \\
\hline-0.1 & -0.31 & -0.4719 & 1.0000 & & \\
\hline EIGENV & VALUES FOLLC & OW. & & & \\
\hline Eigenv & values & As Percenta & Cumu & nul. Percentages & \\
\hline ...... & ....... & $\ldots$ & $\cdots$ & . & \\
\hline & 2.3714 & 59.2856 & & 59.2856 & \\
\hline & 0.8387 & 20.9670 & & 80.2526 & \\
\hline & 0.6032 & 15.0789 & & 95.3315 & \\
\hline & 0.1867 & 4.6685 & & 100.0000 & \\
\hline EIGENV & JECTORS FOLL & LOW. & & & \\
\hline VBLE. & EV-1 & EV-2 & EV-4 & EV-5 & EV-7 \\
\hline ..... & ...... & n.... & - $\quad \ldots .$. & 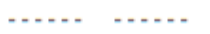 & $\ldots$ \\
\hline 1 & $-.4180 E+00$ & $-.6112 E+00$ & $0.6721 \mathrm{E}+00$ & $-.7697 \mathrm{E}-02$ & \\
\hline 2 & $-.5680 E+00$ & $-.1446 \mathrm{E}+00$ & $-.4772 \mathrm{E}+00$ & $0.6548 \mathrm{E}+00$ & \\
\hline 3 & $-.5928 \mathrm{E}+00$ & $0.6038 E-01$ & $-.3221 \mathrm{E}+00$ & $-.7357 \mathrm{E}+00$ & \\
\hline 4 & $0.3890 E+00$ & $-.7758 \mathrm{E}+00$ & $-.4656 \mathrm{E}+00$ & $-.1732 \mathrm{E}+00$ & \\
\hline PROJEC & CTIONS OF RO & OW-POINTS FOLL & LOW. & & \\
\hline OBJECT & T PROJ-1 $P$ & PROJ-2 PROJ-3 & 3 PROJ-4 P & PROJ-5 PROJ-6 & PROJ-7 \\
\hline .... & ..... & -... & - ...... & .... & ..... \\
\hline 1 & $-.3368 \mathrm{E}-01$ & $-.2051 \mathrm{E}-01$ & $0.7016 \mathrm{E}-02$ & $-.2892 \mathrm{E}-03$ & \\
\hline 2 & $-.2880 E-01$ & $-.1856 \mathrm{E}-01$ & $-.2070 E-02$ & $-.9025 E-02$ & \\
\hline 3 & -.1120 E-01 & $-.2733 \mathrm{E}-02$ & $-.1884 \mathrm{E}-01$ & $0.1111 \mathrm{E}-02$ & \\
\hline 4 & $-.2027 \mathrm{E}-01$ & $0.4075 E-02$ & $-.6164 \mathrm{E}-02$ & $-.1137 \mathrm{E}-01$ & \\
\hline 5 & $-.2354 \mathrm{E}-01$ & $-.1342 \mathrm{E}-02$ & $-.1168 \mathrm{E}-01$ & $0.9689 E-02$ & \\
\hline 6 & $-.1263 E-01$ & $0.1810 \mathrm{E}-01$ & $-.1907 E-01$ & $-.4112 \mathrm{E}-02$ & \\
\hline 7 & $-.2868 \mathrm{E}-01$ & $0.2008 \mathrm{E}-01$ & $-.1773 E-01$ & $0.5555 E-02$ & \\
\hline 8 & -1262 E-01 & $0.9145 \mathrm{E}-03$ & $-.1062 \mathrm{E}-01$ & $-.1013 \mathrm{E}-01$ & \\
\hline 9 & $-.1989 E-01$ & $0.6496 \mathrm{E}-02$ & $-.1309 E-01$ & $-.5333 E-02$ & \\
\hline 10 & $-.2942 \mathrm{E}-01$ & $0.2681 E-03$ & $-.2202 \mathrm{E}-01$ & $0.4526 \mathrm{E}-02$ & \\
\hline 11 & - .1991E-01 & $-.1584 \mathrm{E}-01$ & $-.2020 E-01$ & $0.2530 \mathrm{E}-02$ & \\
\hline 12 & $-.3331 \mathrm{E}-01$ & $0.2448 E-01$ & $-.6046 \mathrm{E}-02$ & $-.1046 \mathrm{E}-01$ & \\
\hline 13 & $-.4847 \mathrm{E}-01$ & $0.1701 E-01$ & $-.1169 \mathrm{E}-01$ & $0.8269 E-02$ & \\
\hline 14 & $-.4484 \mathrm{E}-01$ & $-.1911 E-01$ & $-.2053 \mathrm{E}-01$ & $0.2064 \mathrm{E}-01$ & \\
\hline 15 & $-.3832 \mathrm{E}-01$ & $-.1966 \mathrm{E}-01$ & $-.3004 E-01$ & $0.1050 E-01$ & \\
\hline 16 & $-.3571 \mathrm{E}-01$ & 0.6680 E-02 & $-.2288 \mathrm{E}-01$ & $0.4405 E-02$ & \\
\hline 17 & $-.2805 E-01$ & $0.6948 E-02$ & $-.2452 E-01$ & $0.2861 \mathrm{E}-02$ & \\
\hline 18 & $-.2714 \mathrm{E}-01$ & $-.2342 E-02$ & $-.2657 \mathrm{E}-01$ & $0.6593 E-02$ & \\
\hline 19 & $-3034 \mathrm{E}-01$ & $-.7686 \mathrm{E}-02$ & $-.2914 \mathrm{E}-01$ & $0.6681 \mathrm{E}-02$ & \\
\hline 20 & $-.8371 \mathrm{E}-01$ & $-.4714 \mathrm{E}-01$ & $0.4949 \mathrm{E}-01$ & $0.1074 \mathrm{E}-01$ & \\
\hline 21 & $-.2969 E-01$ & $-.3388 \mathrm{E}-01$ & $-.1778 E-01$ & $-.4792 \mathrm{E}-02$ & \\
\hline 22 & $-.2391 \mathrm{E}-01$ & $-.8969 E-02$ & $-.1637 E-01$ & $0.1889 E-02$ & \\
\hline
\end{tabular}

Figura 3.4: Exemplo de resultado fornecido pela rotina PCA_S3P: matriz de correlação, autovalores, autovetores e projeções de cada píxel nas componentes principais calculadas pela rotina. 


\section{NCC2916}

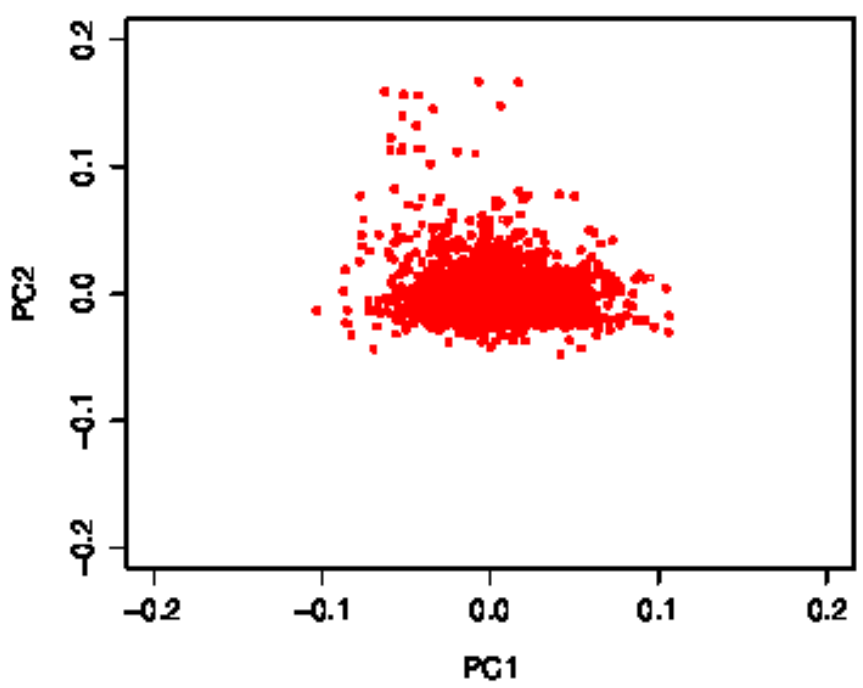

Figura 3.5: Análise PCA nos píxeis: a segunda componente principal em função da primeira componente principal para a galáxia NGC2916.
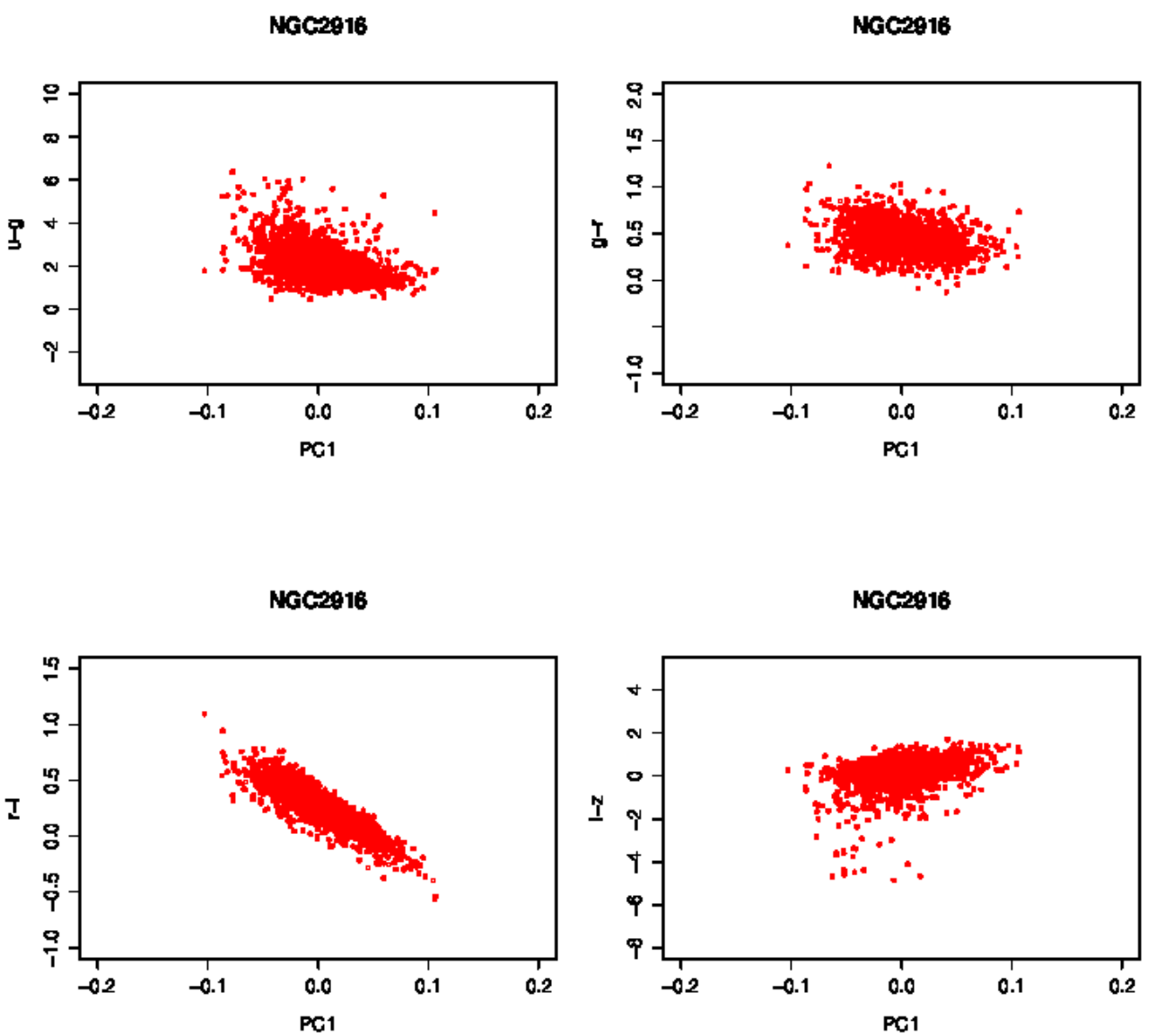

Figura 3.6: Análise PCA nos píxeis: as cores $u-g, g-r, r-i$ e $i-z$ em função da primeira componente principal para a galáxia NGC2916. 


\subsection{Conclusões}

Como visto, a análise de componentes principais (PCA) é um artífico geométrico muito útil na redução de dimensionalidades e visualização das informações de um dado problema. Dada a imensa aplicabilidade deste tipo de análise de dados, principalmente em diversos temas da astronomia, através da aplicação dessa análise aos dados das galáxias do SDDS, mostrou-se que tal técnica é eficiente na caracterização dos tipos espectrais de uma galáxia. A partir dessa constatação, a análise PCA também é efetiva no estudo de populações estelares píxel a píxel, onde a primeira componente principal pode ser tomada como uma proxy das populações estelares em cada píxel da galáxia. 
Capítulo 4

\section{Análise das Imagens das Galáxias}

Neste capítulo, serão apresentados os métodos e procedimentos utilizados durante o pré-processamento e processamento das imagens das galáxias de nossa amostra. Na seção 4.1 é discutida a extração, redução e pré-processamento realizados, passos extremamente importantes da análise. Na seção 4.2 serão apresentadas e explicadas as etapas do processamento das imagens, realizadas pelas rotinas S3P e PCA_S3P, desenvolvidas para este trabalho. Em cada passo da análise, utilizaremos a galáxia NGC2916 (figura 4.1) como exemplo.

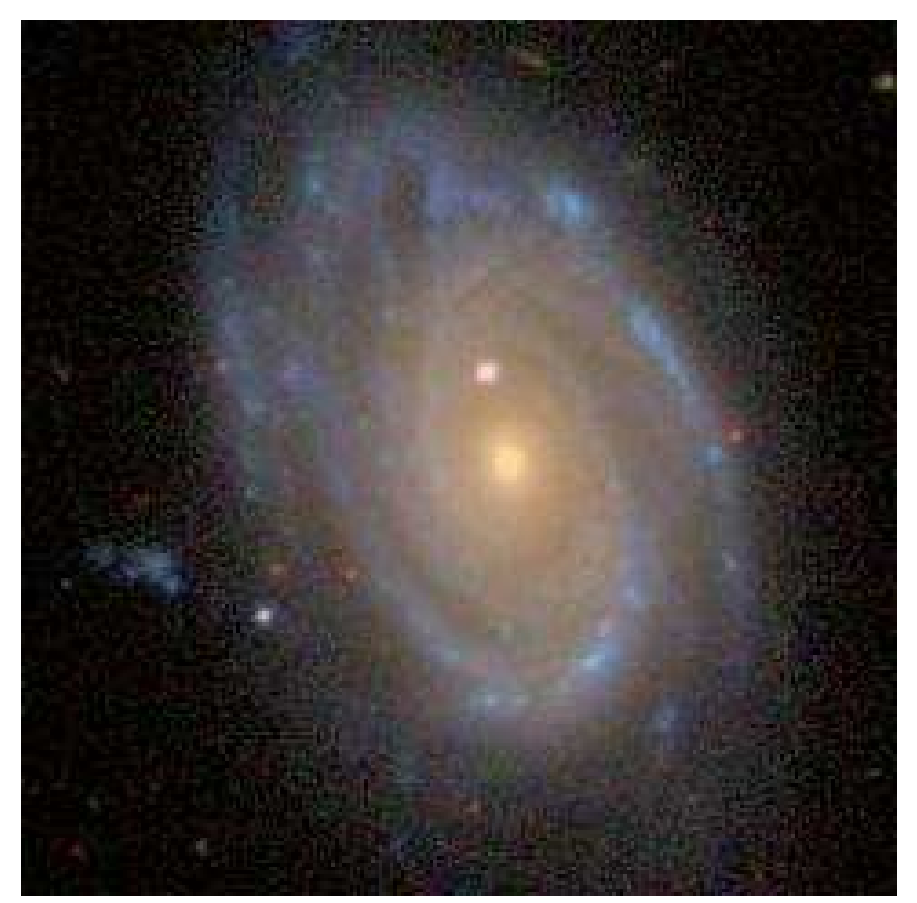

Figura 4.1: Galáxia espiral NGC2916, utilizada para demonstar cada passo do processo. 


\subsection{Extração, Redução e Pré-Processamento dos Dados}

As imagens das 15 galáxias, no formato .fits e contagens nas bandas ugriz, foram obtidos diretamente do SDSS/DR7 através da ferramenta Exploren 1 . De posse desses dados, foi necessário reduzir e pré-processar as imagens antes de aplicarmos as nossas análises. A redução consistiu no registro, limpeza, recorte e listagem, todos descritos abaixo. Estas imagens são, tipicamente, de 2048x1490 píxeis e cada píxel tem 0.396 segundos de arco. A imagem 4.2 exibe a galáxia NGC2916 tal como obtida diretamente pelo SDSS.

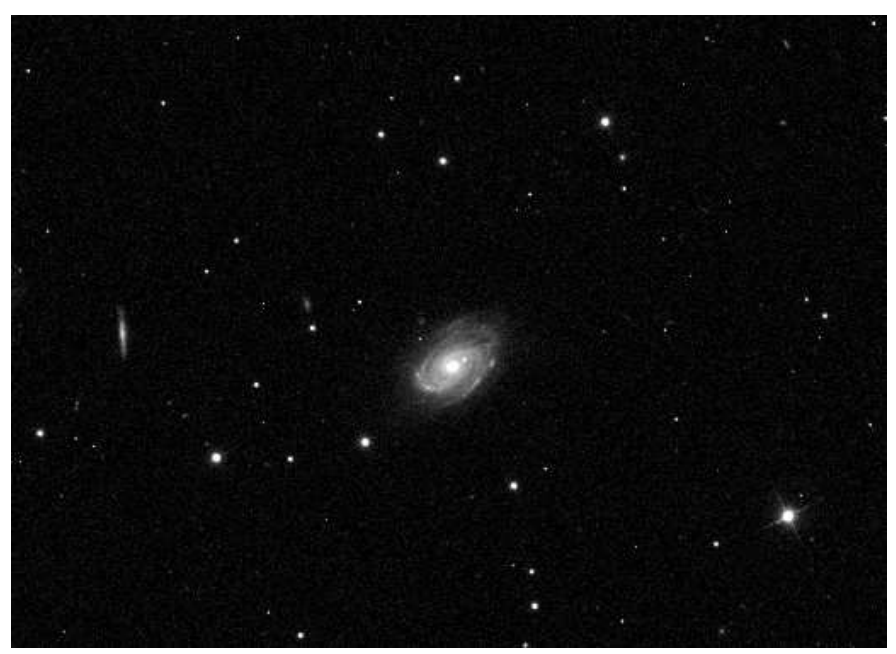

Figura 4.2: NGC2916, na banda $r$, tal como obtida diretamente pelo SDSS.

- Registro: O primeiro passo da redução consistiu em registrar as imagens das galáxias, colocando as imagens nas 5 bandas em um mesmo sistema de coordenadas, de modo que um dado píxel/ponto de uma galáxia, numa dada banda, corresponda ao mesmo em outra banda (a menos, é claro, dos efeitos do seeing). Todas as análises desta etapa foram realizadas considerando a banda $r$ como referência. Para tal tarefa, foram utilizadas as tasks IMSHIFT e XREGISTER do Iraf.

- Limpeza do Foreground: Para eliminar os objetos de foreground que estavam no campo de visão de cada objeto (na maioria dos casos, estrelas da nossa Galáxia), utilizou-se a task FIXPIX do Iraf que, dada uma máscara, substitui as contagens nos píxeis contaminados pela média da sua vizinhança.

\footnotetext{
${ }^{1}$ http://cas.sdss.org/dr7/en/tools/explore/obj.asp
} 
- Recorte: Para diminuir o gasto em tempo computacional, recortamos a imagem original,de modo a ter a galáxia aproximadamente no centro da nova imagem e com menor número de píxeis. Ainda, esta nova imagem deve ser suficientemente grande para permitir não só a análise da galáxia mas também uma estimativa das contagens de fundo de céu. Na figura 4.3 está a galáxia NGC2916 já recortada e com os objetos de foreground eliminados.

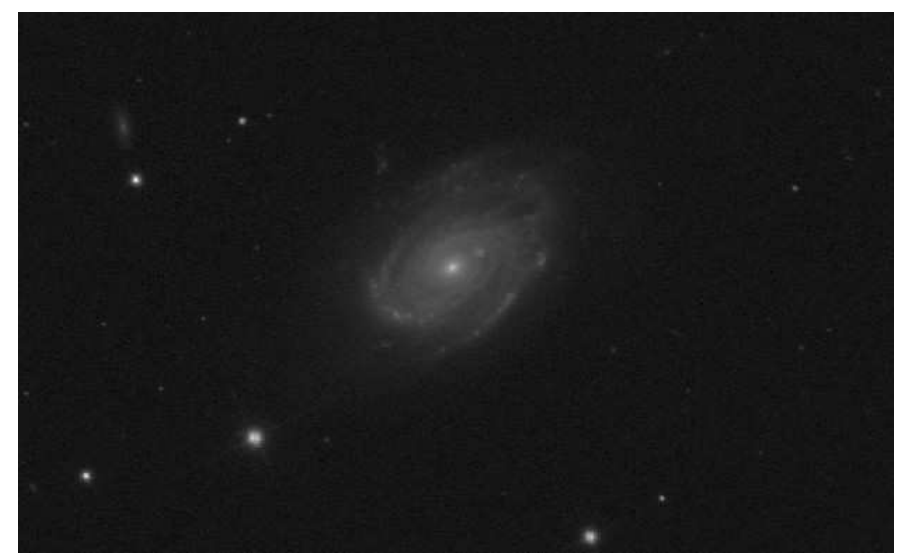

Figura 4.3: NGC2916 após o recorte.

- Listagem: Com a imagem já registrada, limpa e recortada, foi utilizada a task LISTPIX do Iraf para transformar as imagens .fits em um arquivo ASCII com as coordenadas $(\mathrm{x}, \mathrm{y})$ e as contagens de cada píxel em todas as 5 bandas.

\subsection{Processamento}

Para o processamento dos dados de cada objeto, foram criadas duas rotinas, em Fortran 90 e Fortran 77, a saber, a rotina S3P - Stellar Population Pixel by Pixel e a rotina PCA_S3P que calcula as componentes principais de cada objeto, utilizando as cores u-r, g-r, r-i e i-z como proxies para as populações estelares na galáxia.

\subsubsection{S3P}

A rotina $\mathbf{S 3 P}$ realiza as seguintes operações:

- Leitura da imagem: O primeiro passo do programa consiste da leitura do arquivo de texto com os dados nas 5 bandas para cada píxel do objeto. 
- Cálculo de estatísticas do céu: São calculadas as médias, medianas e dispersão do céu em cada banda em uma região definida pelo usuário. Esses dados serão utilizados na segmentação da imagem. Na tabela 4.1 estão as estatísticas do céu para a galáxia NGC2916.

Tabela 4.1 - Estatísticas do céu da galáxia NGC2916: média, mediana e dispersão $\sigma$ das contagens do céu.

\begin{tabular}{lccc}
\hline \hline Banda & Média & Mediana & $\sigma$ \\
\hline$u$ & 1017.7601 & 1018.0 & 3.6337073 \\
$g$ & 1042.9830 & 1045.5 & 25.402922 \\
$r$ & 1072.0702 & 1068.0 & 46.13375 \\
$i$ & 1128.1847 & 1095.5 & 105.19701 \\
$z$ & 1139.4412 & 1130.0 & 96.761375 \\
\hline
\end{tabular}

- Recorte e binagem da imagem: Novo recorte e binagem dos píxeis. A fim de evitar problemas na análise dos dados devido ao seeing característico do SDSS ( 1.5"), binamos a imagem em $4 \mathrm{x} 4$ píxeis $\left(\sim 2.5 \operatorname{arcsec}^{2}\right)$, de modo que em cada novo píxel tenhamos a soma das contagens dos 16 píxeis utilizados na binagem.

- Cálculo da cor: O programa calcula as cores da galáxia em cada píxel segundo as especificações dadas pelo usuário.

- Segmentação e Subtração do céu: Em visão computacional, segmentar imagens significa dividir uma imagem em diversas regiões. Em nossa análise, a segmentação realizada na banda $r$ foi utilizada para determinar os pontos que pertenciam a galáxia e quais eram fundo de céu. A segmentação e a subtração do céu foi realizada considerando como píxeis importantes apenas aqueles que estavam acima de um dado limiar sobre a média do fundo de céu, obtido através de

$$
I_{\text {limiar }}^{\prime}=I_{C}^{\prime}+2.0 \sigma^{\prime}
$$

onde

$$
I_{C}^{\prime}=N_{p i x} I_{C}
$$




$$
\sigma^{\prime}=\sqrt{N_{p i x}} \sigma
$$

com $N_{\text {pix }}$ sendo o número de píxeis usados na binagem, $\sigma$ e $I_{C}$ a dispersão e o fundo de céu da imagem não binada, respectivamente.

Para a galáxia de exemplo, temos que $\sigma_{r}^{\prime}=46.13375$ e $I_{\text {limiar }}^{\prime}=1139.77$. A figura 4.4 mostra a segmentação da galáxia exemplo NGC2916, onde valores 0 representam fundo de céu e valores 1 representam o objeto segmentado.

- Calibração fotométrica: Para a realização da análise PCA é necessário a calibração fotométrica das galáxias a serem estudadas. A magnitude de cada píxel foi calculada a partir da magnitude de Petrosian, medida dentro do raio de Petrosian na banda $r$, $\mathfrak{R}_{P}(r)$. Somando todas as contagens dentro do raio de Petrosian $\mathfrak{R}_{P}(r)$, a constante de calibração foi calculada através de

$$
\text { cte }=\text { mag }_{\text {petro }}+2,5 \log \sum(\text { contagens })
$$

onde o céu não foi incluído por já ter sido subtraído no passo anterior. Com os valores das constantes de calibração em cada banda, as magnitudes em cada píxel, em cada banda, é calculada e já corrigida pelo avermelhamento através de

$$
m a g=-2,5 \log (\text { contagem no pixel })+c t e-A_{\lambda}
$$

onde $A_{\lambda}$ é o avermelhamento da galáxia na banda correspondente. A tabela 4.2 mostra os valores das magnitudes de Petrosian, constantes de calibração e avermelhamento para a galáxia NGC2916, nas cinco bandas.

Tabela 4.2 - Magnitudes de Petrosian, constantes de calibração e avermelhamento para a galáxia NGC2916, nas cinco bandas.

\begin{tabular}{lccc}
\hline \hline Banda & MagPetro & Cte de Calibração & $A_{\lambda}$ \\
\hline $\mathrm{u}$ & 14.87 & 28.43 & 0.136 \\
$\mathrm{~g}$ & 13.19 & 29.55 & 0.100 \\
$\mathrm{r}$ & 12.63 & 29.48 & 0.073 \\
$\mathrm{i}$ & 12.32 & 29.88 & 0.055 \\
$\mathrm{z}$ & 11.97 & 28.048 & 0.039 \\
& & & \\
\hline
\end{tabular}


0000000000000000000000000000000000000000000000000000000000000000000000000000000000000000000000000000 0000000000000000000000000000000000000000000000000000000000000000000000000000000000000000000000000000 0000000000000000000000000000000000000000000000000000000000000000000000000000000000000000000000000000 0000000000000000000000000000000000000000000000000000000000000000000000000000000000000000000000000000 0000000000000000000000000000000000000000000000000000000000000000000100000000000000000000000000000000000 000000000000000000000000001000000000000000000000000000000001111111000000000000000000000000000000000000 000000000000000000000000011000000000000000000000011111111111111111101100000000000000000000000000000 000000000000000000000000001000000000000000000001111111111111111111111111111000000000000000000000000 0000000000000000000000000111000000000000000001111111111111111111111111111111100000000000000000000000 000000000000000000000000011100000000000001111111111111111111111111111111111010010000000000000000000 0000000000000000000000000111100000000000111111111111111111111111111111111111110000000000000000000000 (00000000

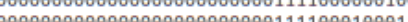
000000000000000000000000111100010001111111111111111111111111111111111111000000000000000000000000

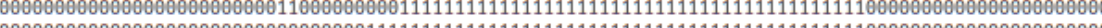

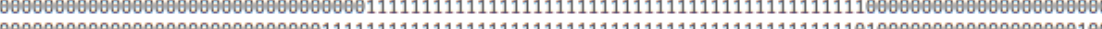
00000000  0000000000000000000000011011111111111111111111111111111111111111111111001000000000000000000 0000000000000000000000000011111111111111111111111111111111111111111111111111111000000000000000000000 000000000000000000000000111111111111111111111111111111111111111111111111111111011100000000000000000 00000000000000000000000111111111111111111111111111111111111111111111111111010100000000000000000000 000000000000000000000010111111111111111111111111111111111111111111111111111000000000000000000000 0000110000000000000001111111111111111111111111111111111111111111111111111111111010000000000000000000000 00000000000000000001111111111111111111111111111111111111111111111111111111011000000000000000000000 00000000000000000000011111111111111111111111111111111111111111111111111111110000000000000000000000 000000000000000000111111111111111111111111111111111111111111111111111111111101000000000000000000000 00000000000000010011111111111111111111111111111111111111111111111111111111111000000000000000000000 000000000000001011111111111111111111111111111111111111111111111111111111111111110100001000000000000000 000000000000001011111111111111111111111111111111111111111111111111111111111111111000000100000000000000 OQ000000000000111111111111111111111111111111111111111111111111111111111111111110000000000000000 0000000000000

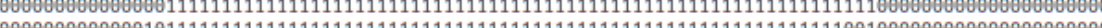
000000000000000000000000000 00000000000011011111111111111111111111111111111111111111111111111110010000000000000000000000 00000000000011111111111111111111111111111111111111111111100000000000000000000000 000000000010111111111111111111111111111111111111111111111000000000000000000000 

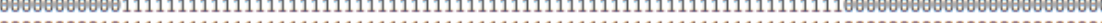

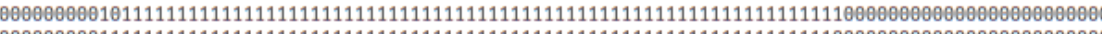
00000000011111111111111111111111111111111111111111111111111111111111111111 000000000000000000000000 000000000011111111111111111111111111111111111111111111111111111111111111111 0000000000000000000000000 0 0 0000000000111111111111111111111111111111111111111111111111111111111111100000000000000000000000000 0000000000110111111111111111111111111111111111111111111111111111111111100000000000000000000000000 0000000000011111111111111111111111111111111111111111111111111111111110000000000000000000000000000 00000000001111111111111111111111111111111111111111111111111111111111110000000000000000000000000000 000001001111011111111111111111111111111111111111111111111111111111111000000000000000000000000000000 00000000101101111111111111111111111111111111111111111111111111111111111100000000000000000000000000000000 0000000001011111111111111111111111111111111111111111111111111111111110000000000000000000000000000 000000  0000000000000000000000 0000000000011111111111111111111111111111111111111111111111111111000000000000000000000000000000000 00000000000111111111111111111111111111111111111111111111111111110000000000000000000000000000000000 0000000000011111111111111111111111111111111110000000000000000000000000000000000 00000000000111111111111111111111110000000000000000000000000000000

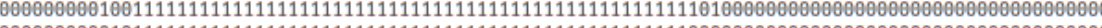
 00001000000101111111111111111111111111111111111111111111111010000000000000000000000000000000000000000 00000000000011101111111111111111111111111111111111111110110000000000000000000000000000000000000000 00000000000101111111111111111111111111111111111111111101000000000000000000000000000110000000000000 0 0000000000000101111111111111111111111111111111111111010000000000000000000000000000000000000000000000000 00000000000010111111111111111111111111111111111111100100000000000000000000000000000000000000000000 00000000000011111111111111111111111111111111111010000000000000000000000000000000000000000000000000 0000000000000001111111111111111111111111111110000000000000000000000000000000000000000000000000000000 0000000000000001111111111111111111111111100000000000000000000000000000000000000000000000000000000 00000000000000001000111110111111111111110000000000000000000000000000000000000000000000000000000000000000 000000000000000000011110010010111110000000000000000000000000000000000000000000000000000000000000 (1) 0 00000000000

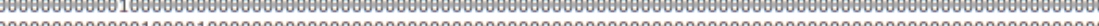

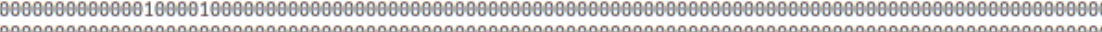
00000000000 00000000000000000000000000000000000000000000000000000000000000000000000000000000000000000000000000000

Figura 4.4: Mapa de Segmentação da galáxia NGC2916, onde o objeto segmentado está representado pelo valor 1 .

- Identificação de objetos (FoF): Para identificar os objetos obtidos pela segmentação anterior, a rotina utiliza o algoritmo Friends of Friends, onde um objeto é definido como um conjunto de píxeis conexos. Nessa etapa, a rotina identifica os objetos e, opcionalmente, imprime um mapa com o objeto detectado, sendo que cada píxel desse objeto (galáxia) será identificado através do número 2 e os demais píxeis recebem 0 . 


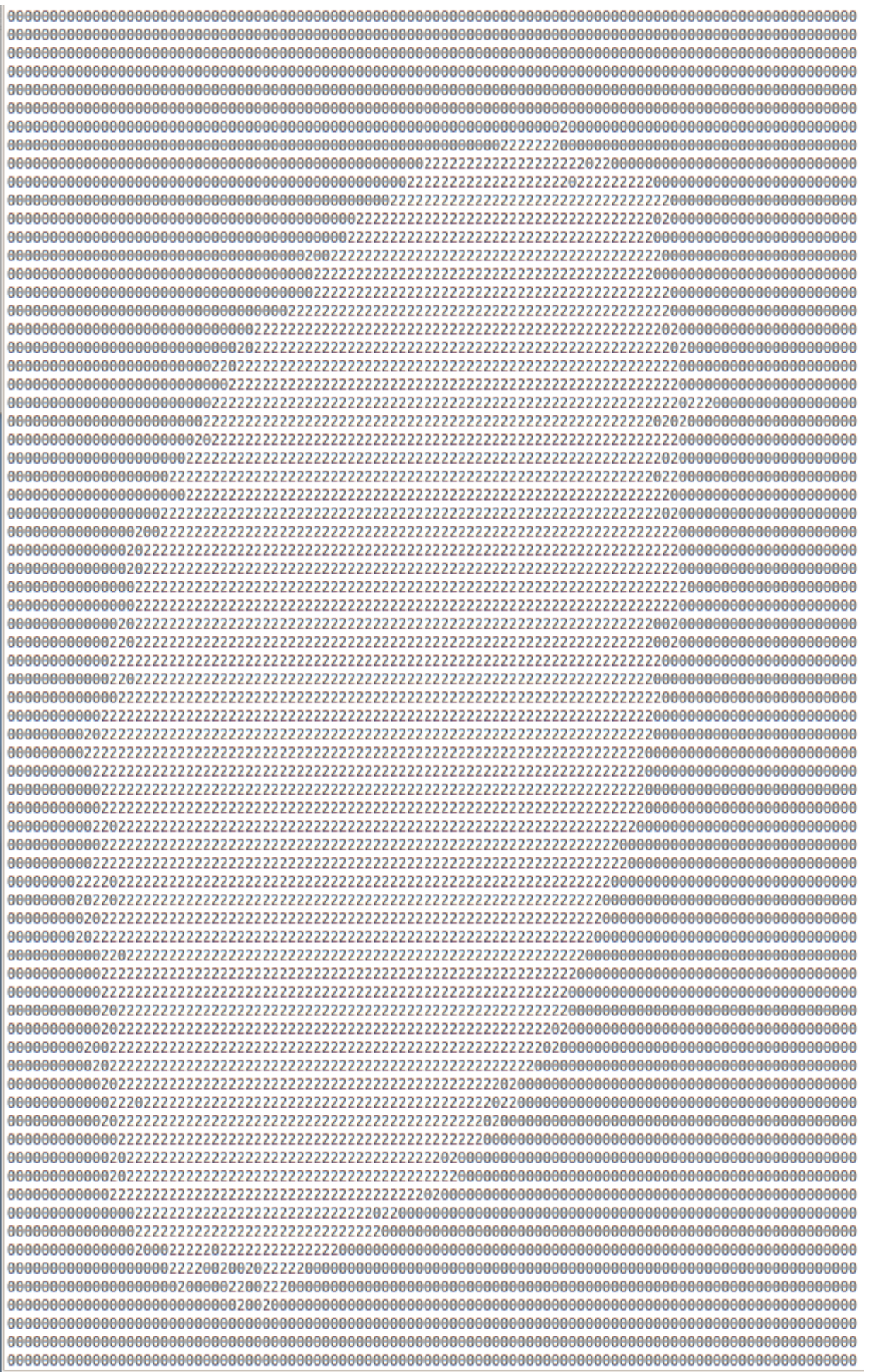

Figura 4.5: Mapa com o objeto identificado via FoF, no exemplo a galáxia NGC2916.

A figura 4.5 mostra um exemplo do mapa criado através da aplicação do algoritmo FoF.

- Identificação dos bins de cores: Utilizando a cor $g-r$ em cada píxel, os píxeis foram divididos em 4 bins de cor, onde cada bin possui o mesmo número de píxeis. Uma vez que uma dada população estelar está correlacionada com suas cores, separar os 


\begin{abstract}
1000000000000000000000000000000000000000000000000000000000000000000000000000000000000000000000000000 1000000000000000000000000000000000000000000000000000000000000000000000000000000000000000000000000000 1000000000000000000000000000000000000000000000000000000000000000000000000000000000000000000000000000 1000000000000000000000000000000000000000000000000000000000000000000000000000000000000000000000000000 1 1000000000000000000000000000000000000000000000000000000000000000000000000000000000000000000000000000 1 0000000000000000000000000000000000000000000000000000000000000000000000000000000000000000000000000 ${ }_{1}$

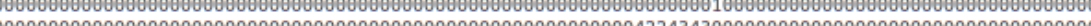
(1)

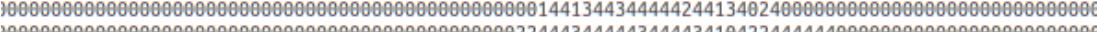
(1)

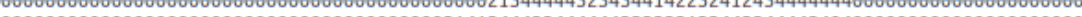

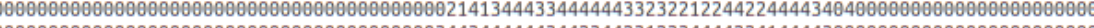
1000000000000000000000000000000000000000344344444434423442312334444224144443000000000000000000000000 1000000000000000000000000000000000010022114411444443344342123412344444123212200000000000000000000000  1000000000000000000000000000000000004433334444434443334433443444444444332342310000000000000000000000 1000000000000000000000000000000004444443444443324344222444343433433444344442310000000000000000000000 1000000000000000000000000000001212244444233444444334332244443244434343444444242101000000000000000000000 00000000000000000000000000401214434233334434444443333334433344334444443444331340100000000000000000000 000000000000000000000004401143222433333333344443323343343344444444433434341122000000000000000000000 00000000000000000000000003121244333444331334444334344332343444344434434442144334000000000000000000000 0000000000000000000000032134321432244222222444332243223443444444444444444111124013100000000000000000 100000000000000000000001344141122222222212334343333232323444334444444444313120101000000000000000000000 jo00000000000000000000103334311121241433334433332332222324443322223444444441211110000000000000000000000 1000000000000000000003242432212312213323433322122221123233323212244444431322101000000000000000000000 (1)

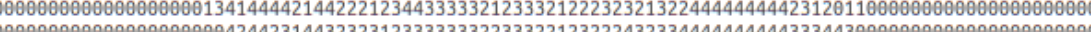
1000000000000000000004244231443232312333333322333221232224323344444444443334430000000000000000000000 0000000000000000013124342322222112333322332233322111221223344444444442223342301000000000000000000000

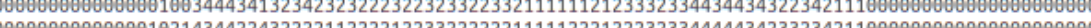

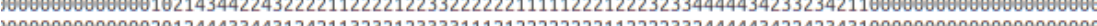
(1) 00000000000000234233314221133232321222223222222221111122232232444443321213111100000000000000000000 1000000000000033434243211223233322222212222211111111111112233234443432122114100000000000000000000 000000000000102414334412121233321222331212111111111111111111233344433331441001000000000000000000000  000000000001214414344133213443221232221111111111111111111122223324444222434100000000000000000000000 30000000000014041243433232234332222221111111111111111111111112233344443333231000000000000000000000000 0000000000002321132434322332431222211111111111111111111111212343444444334444100000000000000000000000 10000000000222 44444344323234321112221111111111111111111111222233344443434444400000000000000000000000000 000000003031234434322343344221112221111111111111111112111122223333434342444000000000000000000000000 0000000014441344243323323432211121111111111111111111111111213333333444413440000000000000000000000000 000000000433423324443243233211122111111111111111111111122222333334444311140000000000000000000000000 000000000041224442444333233112111111111111111111111111111222343223333322110000000000000000000000000 0000000000114444223343343332222111111111111111111111121112223332424222121100000000000000000000000000 000000000310423434333344333212211111111111111111122211112222423233212433100000000000000000000000000 1000000

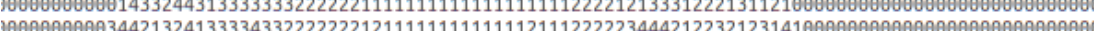
(1) 1000000014120322132122334322212111111111111111121112222223 344222114411300000000000000000000000000000 0000000402304243413333332221221111111111123222211232224441121111344000000000000000000000000000000

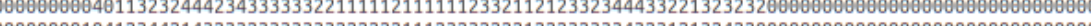
(1)

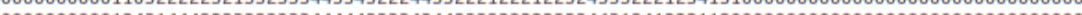

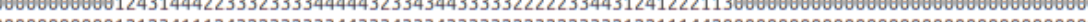

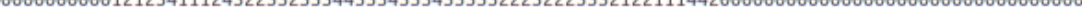
000000000010211332111111122233433223333322123223333343232242133310000000000000000000000000000000000 000000000020323324231411123232222222332221132223344343123134422010000000000000000000000000000000000 000000001004442214433411212221221223332322122213343343214114130100000000000000000000000000000000000 0000000001013312213221222233221223223222222222233333222411432300000000000000000000000000000000000000 00000000001033144444333233443313324322223422334333244322204000000000000000000000000000000000000000000 00000000000423014121334344444132424343213432223212243344044000000000000000000000000000000000000000000 000000000020241421313142444442333423433342333331243432401000000000000000000000000000000000000000000 000000000000331442444422444331223212334442432423141431100000000000000000000000000000000000000000000  000000000001041412113124224233344444244423311211113100000000000000000000000000000000000000000000000 1000000000003334124244443332324423443121122312313030000000000000000000000000000000000000000000000000 000000000000314334424143343442442121114201100000000000000000000000000000000000000000000000000000

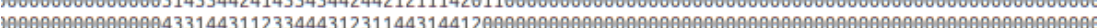
(1) (0) (1)

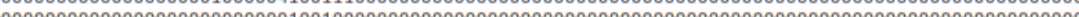
1000000000000000000000000000000000000000000000000000000000000000000000000000000000000000000000000000
\end{abstract}

Figura 4.6: Mapa de Distribuição espacial de cor, no exemplo a galáxia NGC2916.

píxeis em 4 bins traz informações preliminares interessantes a respeito das populações estelares de uma galáxia.

- Mapa das cores: Com os píxeis divididos em 4 grupos de cores, é possível analisar a distribuição espacial das mesmas, utilizando um conjunto de estimadores estatísticos. A rotina também exibe um mapa para as populações, onde os píxeis associados a SP 
mais azuis recebem o valor 1 , os píxeis associados a SP mais vermelhas recebem o valor 4 e os intermediários recebem 2 e 3. Além do mapa, que é apenas um auxiliar para averiguar os resultados do processamento, também são gerados arquivos de texto com as populações. Na figura 4.6, está um exemplo de mapa de distribuição espacial de populações gerado pela rotina S3P.

- Identificação das populações: Com as magnitudes medidas em cada píxel, fazemos uma PCA usando o programa PCA_S3P e associamos a cada píxel um tipo espectral definido pelo valor da primeira PCA das magnitudes associadas aquele píxel. Em seguida, dividimos a primeira PCA em 4 bins, atribuindo uma população a cada bin, analogamente ao que fizemos com as cores na etapa anterior.

- Análise das populações: Após a binagem, segmentação, recorte, identifição dos objetos e definição das populações, foi realizada então uma análise morfológica mais elaborada de cada objeto. Os itens dessa análise estão descritos no próximo capítulo.

Assim sendo, como produtos da rotina S3P, são gerados arquivos de texto com os quatro grupos de cores, com as contagens em cada novo píxel da galáxia, a cor g-r, e um último arquivo com todas as informações a respeito do processamento, com os dados do céu, da binagem e segmentação na banda r, mapas de segmentação, mapa de estruturas identificadas pelo método FoF, mapa de populações de cores, além dos parâmetros morfológicos que serão apresentados no próximo capítulo. O programa PCA_S3P produz um arquivo com os píxeis e as componentes principais associadas a cada píxel. 
Capítulo 5

\section{Estimadores Estatísticos da Distribuição de Populações Estelares}

A descrição morfológica de um objeto requer tanto características topológicas quanto geométricas. Na prática, é interessante ter tantas informações quanto possível expressas em termos de alguns parâmetros robustos e significativos.

Para analisar a morfologia de uma dada população estelar (SP) em uma galáxia, primeiramente é criada uma imagem binária, com intensidades iguais a 1 (píxeis brancos) para os píxeis pertencentes a uma dada população e com intensidade igual a 0 (píxeis pretos) no caso contrário.

Os estimadores estatísticos discutidos nas próximas sessões são aplicados nessas imagens binárias, onde caracterizamos um objeto como sendo um conjunto de píxeis brancos associados a uma dada população.

Assim, produzimos um conjunto de quantidades estatísticas para descrever e quantificar a morfologia de cada população estelar nas galáxias. Estas quantidades são:

- Raio médio e dispersão;

- Coeficiente de Gini;

- Funcionais de Minkowski.

Cada uma dessas estatísticas serão descritas a seguir. 


\subsection{Raio Médio e Dispersão}

Se $\left(x_{i}, y_{i}\right), i=1, \ldots, N$ é o conjunto de píxeis associados com uma dada $S P$, seu centro é definido como $(\bar{x}, \bar{y})$ onde

$$
\bar{x}=\frac{1}{N} \sum_{i=1}^{N} x_{i} \quad \bar{y}=\frac{1}{N} \sum_{i=1}^{N} y_{i},
$$

o raio médio será dado por

$$
\bar{R}=\frac{1}{N} \sum_{i=1}^{N} R_{i}
$$

onde

$$
R_{i}=\left(\left(x_{i}-\bar{x}\right)^{2}+\left(y_{i}-\bar{y}\right)^{2}\right)^{1 / 2},
$$

e a dispersão do raio definida como

$$
\sigma_{R}=\frac{1}{N-1} \sum_{i=1}^{N}\left(R_{i}-\bar{R}\right)^{1 / 2} .
$$

Pode ser útil normalizar estas quantidades pelo o raio equivalente: se $N_{T}$ é o número de píxeis do objeto inteiro (i.e., contendo todas as populações), o raio equivalente é definido como

$$
R_{E}=\left(\frac{N_{T}}{\pi}\right)^{1 / 2} .
$$

Normalizados dessa forma, os raios médios são uma medida da concentração de cada população.

\subsection{Coeficiente de Gini}

O Coeficiente de Gini é uma medida de dispersão estatística, proposta pelo estatístico e sociólogo italiano Corrado Gini (Gini, 1912). Utilizado principalmente em estudos na área de Economia, para quantificar distribuição de renda ou riquezas, o Coeficiente de Gini (a partir daqui apenas CG) mede as desiguldades em uma distribuição de frequências. O CG é calculado de tal modo que uma distribuição completamente igualitária tem $C G=0$ e uma distribuição completamente concentrada tem $C G=1$. Dado um conjunto de dados $X_{i}$, o CG pode ser definido e calculado como a média da diferença absoluta entre todas as combinações de $X_{i}$, dada pela equação 5.1. Glasser (1962), propôs um modo mais fácil e eficiente de calcular o CG, através de uma somatória dos $X_{i}$ ordenados na forma apresentada em 5.2 . 


$$
\begin{aligned}
& C G=\frac{1}{2 \bar{X} n(n-1)} \sum_{i=1}^{n} \sum_{j=1}^{n}\left|X_{i}-X_{j}\right| \\
& C G=\frac{1}{\bar{X} n(n-1)} \sum_{i=1}^{n}(2 i-n-1) X_{i}
\end{aligned}
$$

Abraham et al. (2003) mostrou que o Coeficiente de Gini da contagem nos píxeis da imagem possui uma correlação com a concentração de luz nas galáxias, podendo ser utilizado como um parâmetro útil em estudos sobre a morfologia das galáxias.

\subsection{Funcionais de Minkowski}

Os Funcionais de Minkowski são ferramentas extremamente úteis em análises de imagens, morfologia matemática e geometria integral, uma vez que quantificam padrões morfológicos nas imagens (Santalo, 1976; Rosenfeld e Kak, 1976; Serra, 1982; Mecke, 1993)). A vantagem do uso dos Funcionais de Minkowski (a partir daqui apenas FM) é que essas medidas topológicas e geométricas podem ser aplicadas efetivamente em imagens binárias (Mecke, 1996; Rehse, 2008).

Em particular, em análises morfológicas de imagem, os FM são ferramentas úteis para a caracterização das imagens, tendo sido utilizado em astronomia, no estudo das estruturas em larga escala (Hikage et al., 2003; Costa-Duarte et al., 2011).

Em duas dimensões, os FM de um objeto são a área da superfície, o perímetro total e a característica de Euler-Poincaré (ou conectividade, número de regiões independentes conectadas menos o número de buracos em cada região). Assumindo que temos uma imagem binária com $N$ píxeis, onde $N_{w}$ é o número de píxeis "brancos" (membros da SP considerada) e $N_{b}$ é o número de píxeis "pretos"(abaixo do limiar),

$$
N=N_{w}+N_{b}
$$

Caracterizando um objeto como sendo um conjunto de píxeis associados a uma certa SP, os FM normalizados de cada objeto podem ser definidos como sendo:

- área da superfície:

$$
A=\frac{N_{w}}{N}
$$


- perímetro:

$$
P=\frac{N_{b w}}{N}
$$

onde $N_{b w}$ representa o número de píxeis na fronteira branco-preto;

- Característica de Euler-Poincaré (CEP): número de regiões independentes conexas menos o número de buracos em cada região)

$$
\begin{gathered}
\chi_{a b s}=C_{w}-C_{b} \\
\chi_{n o r m}=\frac{C_{w}-C_{b}}{N}
\end{gathered}
$$

onde $C_{w}$ e $C_{b}$ são o número de conjuntos contínuos de píxeis brancos e pretos, respectivamente. A primeira equação corresponde a CEP absoluta e a segunda equação corresponde a CEP normalizada pelo número de píxeis da imagem.

Dentre os três Funcionais de Minkowski definidos acima, neste trabalho, será utilizado apenas o último, a Característica de Euler-Poincaré, sendo que os demais funcionais será utilizado em trabalhos futuros.

No próximo capítulo, vamos aplicar estes estimadores estatísticos às populações estelares identificadas nas galáxias para quantificar a morfologia dessas populações. 
Capítulo 6

\section{Resultados}

Neste capítulo serão apresentados os resultados da análise de populações estelares num approach píxel a píxel, através da análise PCA e de alguns estimadores estatísticos em cada píxel da galáxia. Na seção 6.1 será realizada a caracterização das populações estelares através da análise PCA e a determinação das 4 populações estelares que serão utilizadas nesse estudo. Na seção 6.2 serão expostos os resultados da análise da distribuição das populações estelares nas galáxias da amostra e nas seções 6.3, 6.4, 6.5 serão apresentados os resultados a respeito da determinação dos raios médios e cores, Coeficiente de Gini e Característica de Euler-Poincaré, respectivamente, das populações estelares.

Com a finalidade de deixar este capítulo mais claro, utilizaremos uma galáxia de cada sub-amostra (elíptica: NGC6125; espiral: NGC4185; espiral tardia: NGC3991) para exemplificar as análises, sendo que as imagens e gráficos para as demais galáxias estarão no apêndice $\mathrm{A}$

\subsection{PCA e Populações Estelares Píxel a Píxel}

Como apresentado no capítulo 3, a análise PCA é uma ferramenta de amplo uso em diversas áreas da astronomia e que tem um grande potencial no estudo de tipos espectrais e populações estelares. Assim como apresentado na seção 3.3, a primeira componente principal calculada para diferenças entre a magnitude $i$ e as demais magnitudes $(u-i, g-i$, $r-i$ e $z-i) 1$ dos píxeis de uma galáxia possui uma correlação muito boa com a população estelar dos mesmos. Ainda, nessa mesma seção [3.3, tomando a galáxia NGC2916 como exemplo, foram apresentados gráficos com a correlação entre a primeira e segunda compo-

\footnotetext{
${ }^{1}$ Aqui, estamos referenciando como magnitudes as contagens, com o céu subtraído e calibradas como explicitado na seção 4.2.1 de modo que a PCA calculada seja proporcional ao logaritmo do fluxo no píxel.
} 
nentes principais, além da correlação entre a primeira componente principal e as cores em cada píxel. Nas figuras A.5, A.10 e A.15, apresentamos as correlações entre as cores e a primeira componente principal para todas as galáxias elípticas, espirais e espirais tardias, respectivamente de nossa amostra.
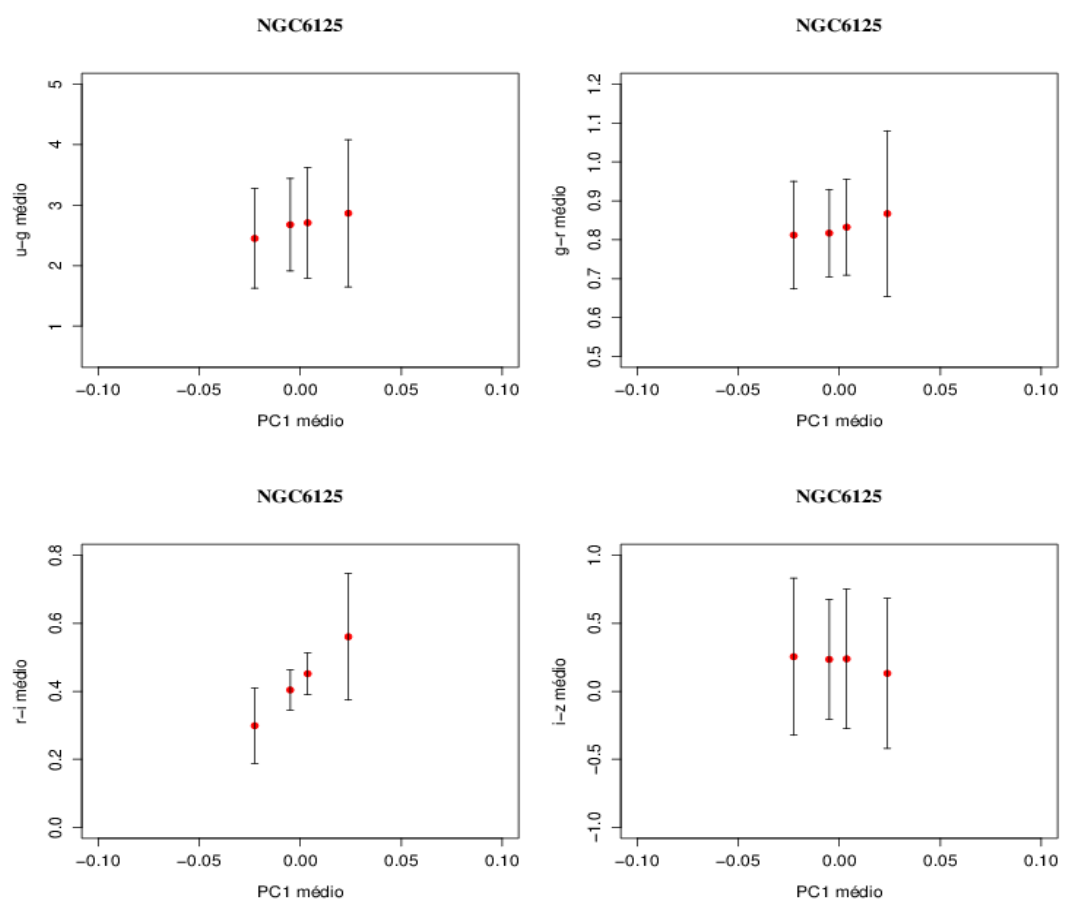

Figura 6.1: Análise PCA nos píxeis: As cores $u-g, g-r, r-i$ e $i-z$ em função da $1^{a}$ componente principal, para NGC6125.

Em todos os casos a correlação entre a primeira componente principal e as cores $u-g$ e $i-z$ possui uma dispersão mais alta do que as demais. Isso acontece, principalmente, porque as galáxias da amostra possuem menor emissão nas bandas $u$ e $z$ em comparação com as demais bandas e, assim, suas imagens nas bandas $u$ e $z$ são mais ruidosas. Dessa forma, quando as galáxias são segmentadas, utilizando como referência a banda $r$, as contagens nas regiões periféricas, nessas bandas, ficam mais incertas. Em nossa análise, foi decidido utilizar a cor $r-i$ para correlacionar com as medidas de raios e componentes principais, pois a mesma apresentou correlação mais significativa com a primeira componente principal. 

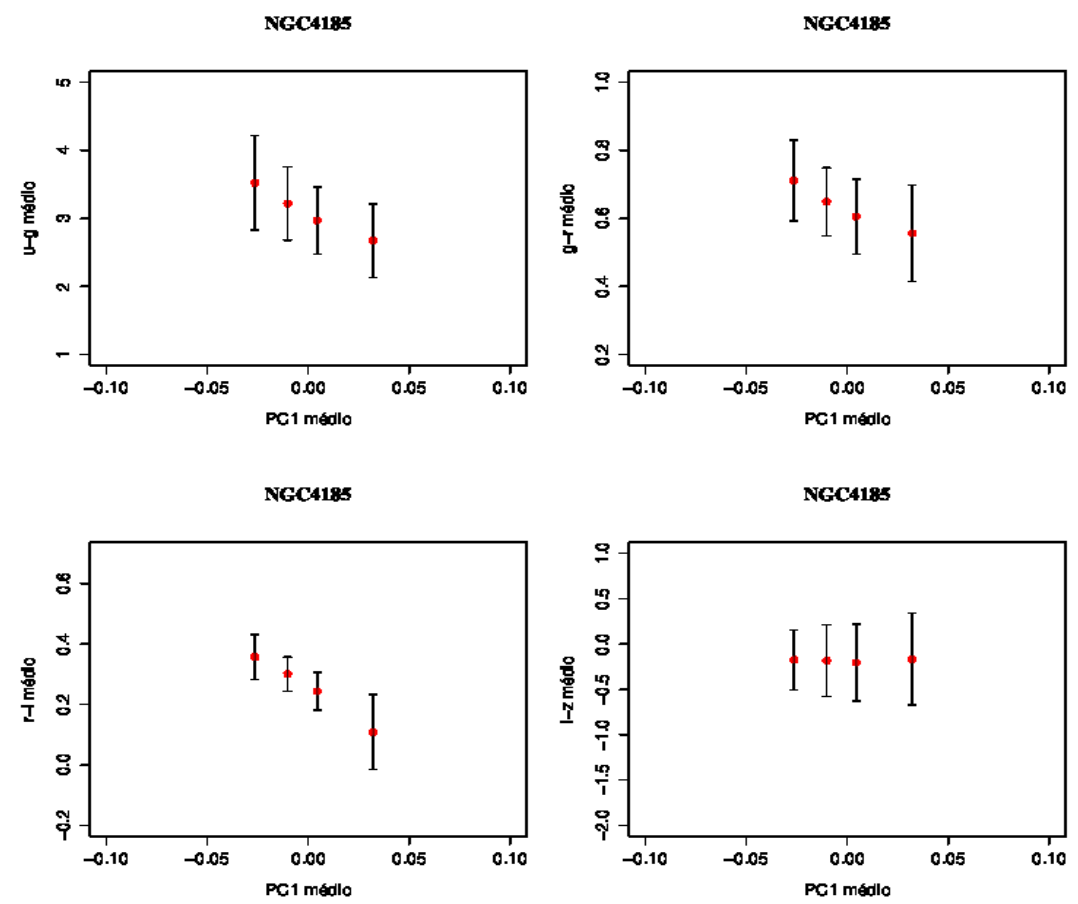

Figura 6.2: Análise PCA nos píxeis: As cores $u-g, g-r, r-i$ e $i$ - $z$ em função da $1^{a}$ componente principal, para NGC4185.

Além das várias propriedades estatísticas que serão abordadas neste trabalho, o principal foco do estudo é compreender como se distribuem espacialmente as populações estelares dentro de uma galáxia e entender como tais distribuições podem ajudar a interpretar a evolução dessas galáxias. Dado esse ponto de vista, utilizaremos a primeira componente principal, calculada via análise PCA nos píxeis das galáxias, como um proxy das populações estelares. Dada a arbitrariedade do sentido das componentes principais, convencionamos que quanto menor (maior) o PC1 mais vermelha (mais azul) será a população. Para entender como as mesmas se distribuem, iremos ordenar de modo crescente a primeira componente principal e dividi-la em 4 bins com o mesmo número de píxeis. Cada bin será referenciado como uma das 4 populações estelares a serem analisadas. O bin com menores valores da primeira componente principal será a população estelar 1 (P1), o segundo bin será a população 2 (P2), o terceiro bin será a população 3 (P3) e, por fim, o bin com os maiores valores da primeira componente principal será referenciado como população 4 $(\mathrm{P} 4)$.

Nas seções a seguir iremos analisar cada população estelar em si e também no contexto da galáxia como um todo. 

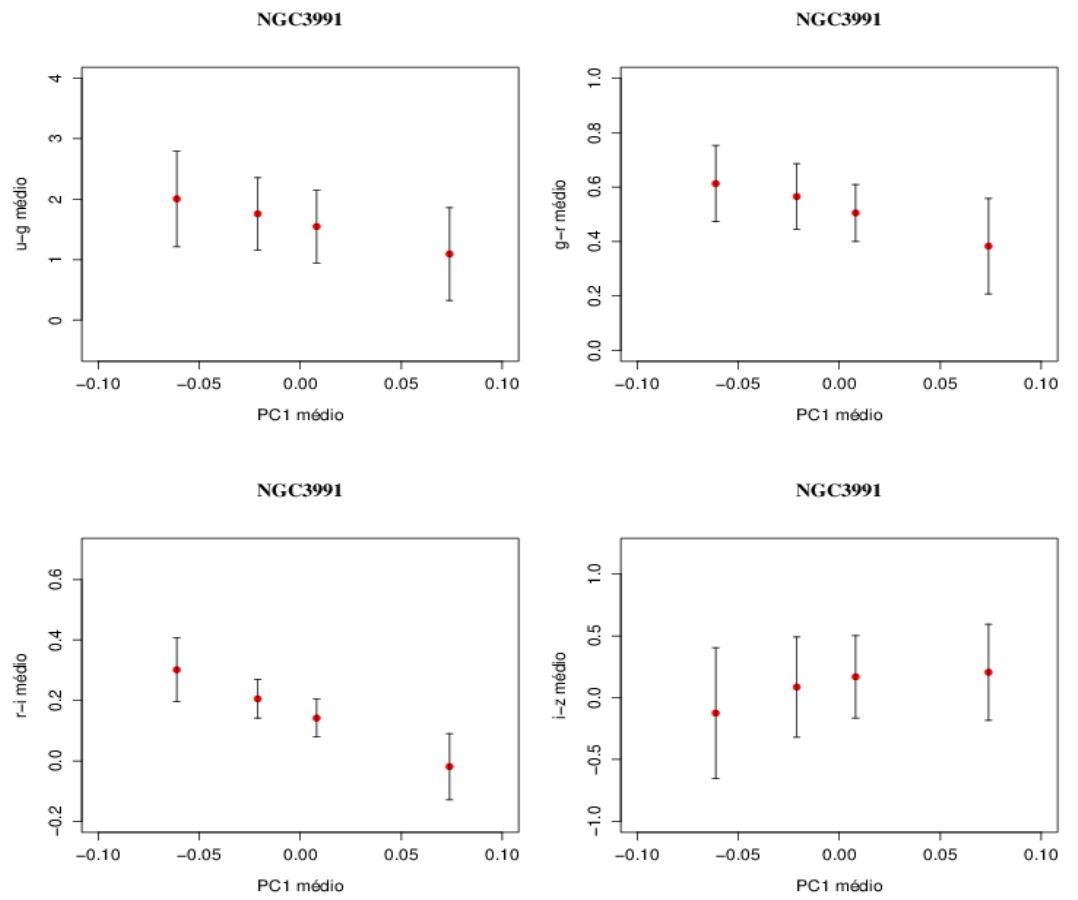

Figura 6.3: Análise PCA nos píxeis: As cores $u-g, g-r, r-i$ e $i$ - $z$ em função da $1^{a}$ componente principal, para ngc3991.

\subsection{Distribuição Espacial de Populações Estelares}

Dentro do estudo do processo de evolução de galáxias, uma das questões chaves é entender como tais objetos se formam e evoluem, tanto do ponto de vista espacial quanto temporal. Nesse sentido, as populações estelares são ferramentas de extrema importância, uma vez que podem ser entendidas como registros fósseis dos processos que tiveram lugar ao longo da evolução da galáxia. Ainda que não seja possível determinar suas posições anteriores (tanto devido a sua evolução passiva quanto a efeitos ambientais, fusões, acresção de gás e outros) e, consequentemente a evolução dos seus movimentos, é possível estudar sua evolução através das relações entre suas atuais posições, suas cores, idades, metalicidades e outras propriedades.

Para entender como evoluem as populações estelares dentro das galáxias, foram analisadas as distribuições espaciais de cada uma das 4 populações estelares (P1, P2, P3 e P4) nas galáxias da amostra. Na figura 6.4 são apresentadas as distribuições espaciais das populações estelares de todas as galáxias da amostra, onde a população 1 é representada pela cor vermelha, a população 2 pela cor amarela, a população 3 pela cor verde e, por fim, a população 4 pela cor azul. 

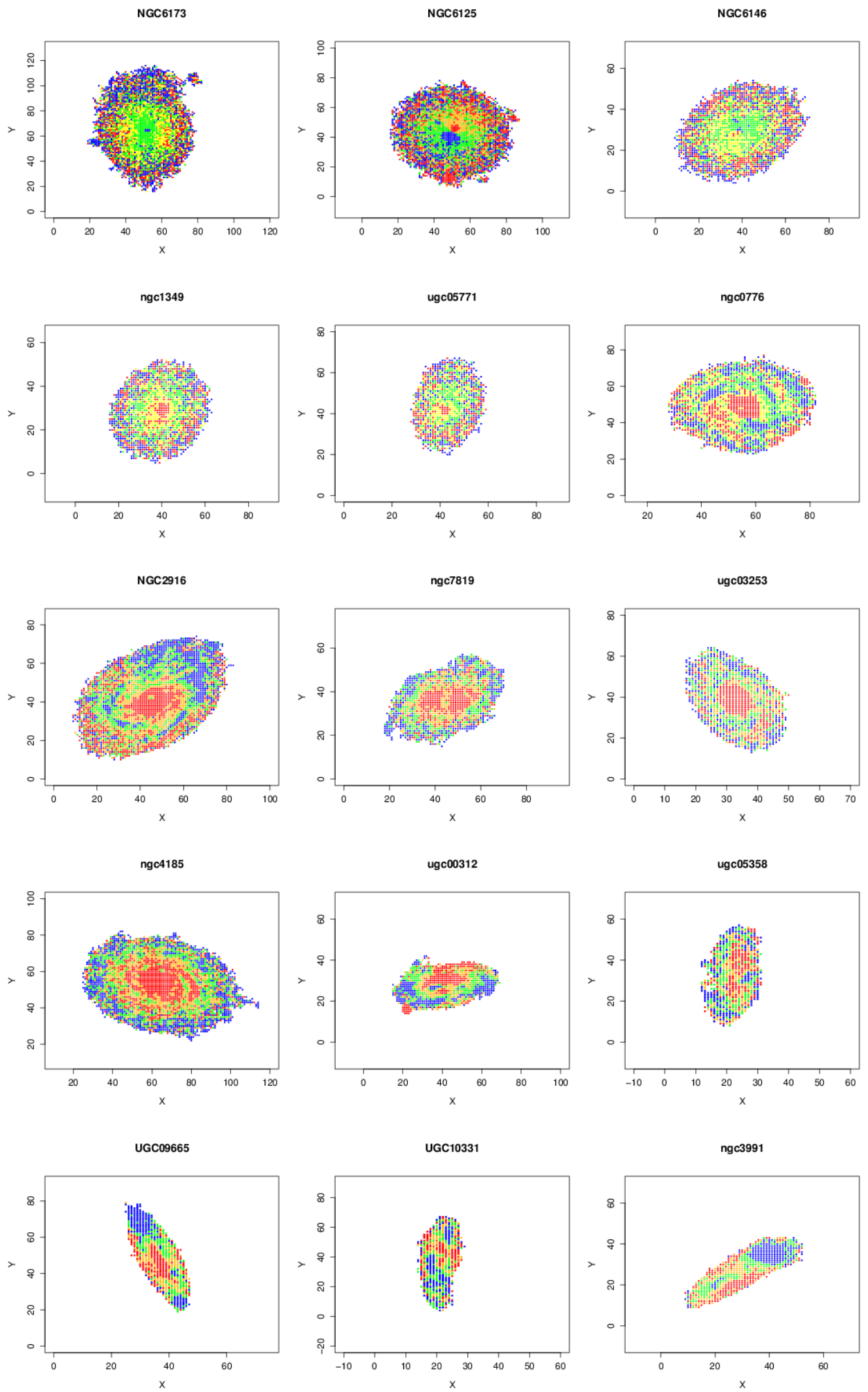

Figura 6.4: Distribuição espacial das populações estelares. 
Nas figuras 6.5, 6.6 6.7 estão detalhadas as distribuições espaciais das galáxias NGC6125, NGC4185 e NGC3991. As distribuições de cada população para as demais galáxias estão apresentadas em A.2.

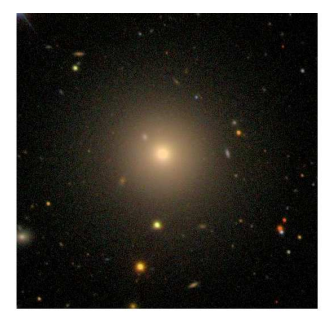

Pop.1

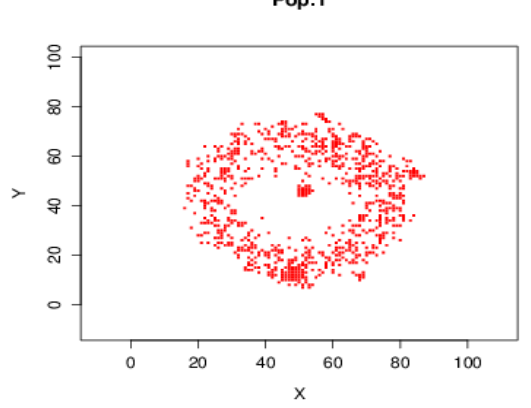

Pop.3

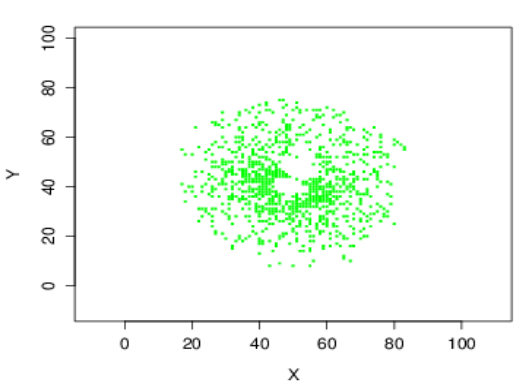

NGC6125

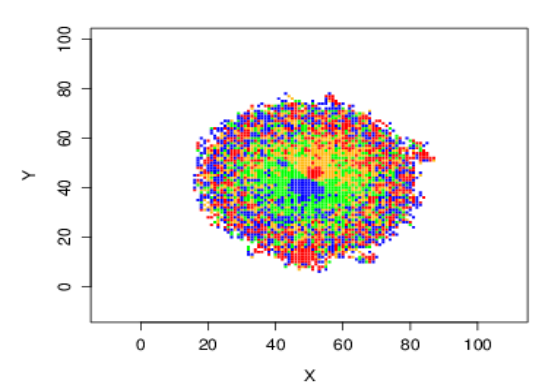

Pop.2

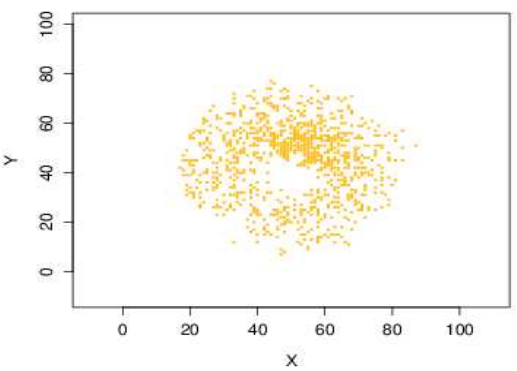

Pop.4

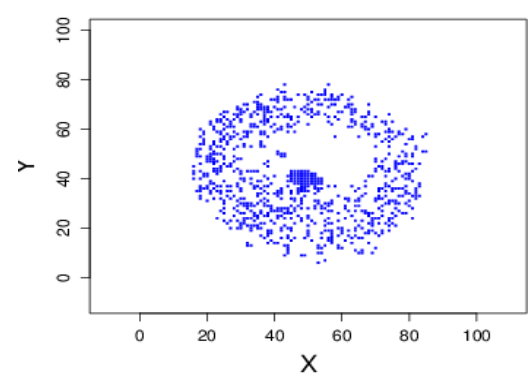

Figura 6.5: Distribuição espacial das populações estelares.

A galáxia elíptica NGC6125 exibe uma distribuição espacial de populações estelares onde as populações mais velhas e as mais novas estão presentes nas regiões externas, enquanto que as populações intermediárias estão distribuídas pela extensão da galáxia. Dado que as galáxias elípticas, tais como a NGC6125, são objetos velhos podemos supor que essas galáxias podem ter sofrido eventos de minor mergers, os quais afetaram sua população estelar, "rejuvenecendo"sua população ao longo da galáxia, além de formação estelar na região periférica. A presença de uma pequena região azul no centro da galáxia pode ser 
um efeito de contaminação devido a presença de uma estrela brilhante nessa região, apesar da limpeza de foreground. Essa mesma tendência é observada nas galáxias NGC6173, NGC6146 e UGC05771. No entanto, na galáxia NGC1349 nota-se que a população mais velha está presente em toda a galáxia, com uma concentração na região interna, as populações intermediárias distribuídas pela galáxia e, por fim, a população mais azul está na região externa da galáxia. Ao contrário das demais elípticas, a NGC1349 parece não ter sofrido efeitos ambientes e/ou de minor mergers recentes e notáveis.
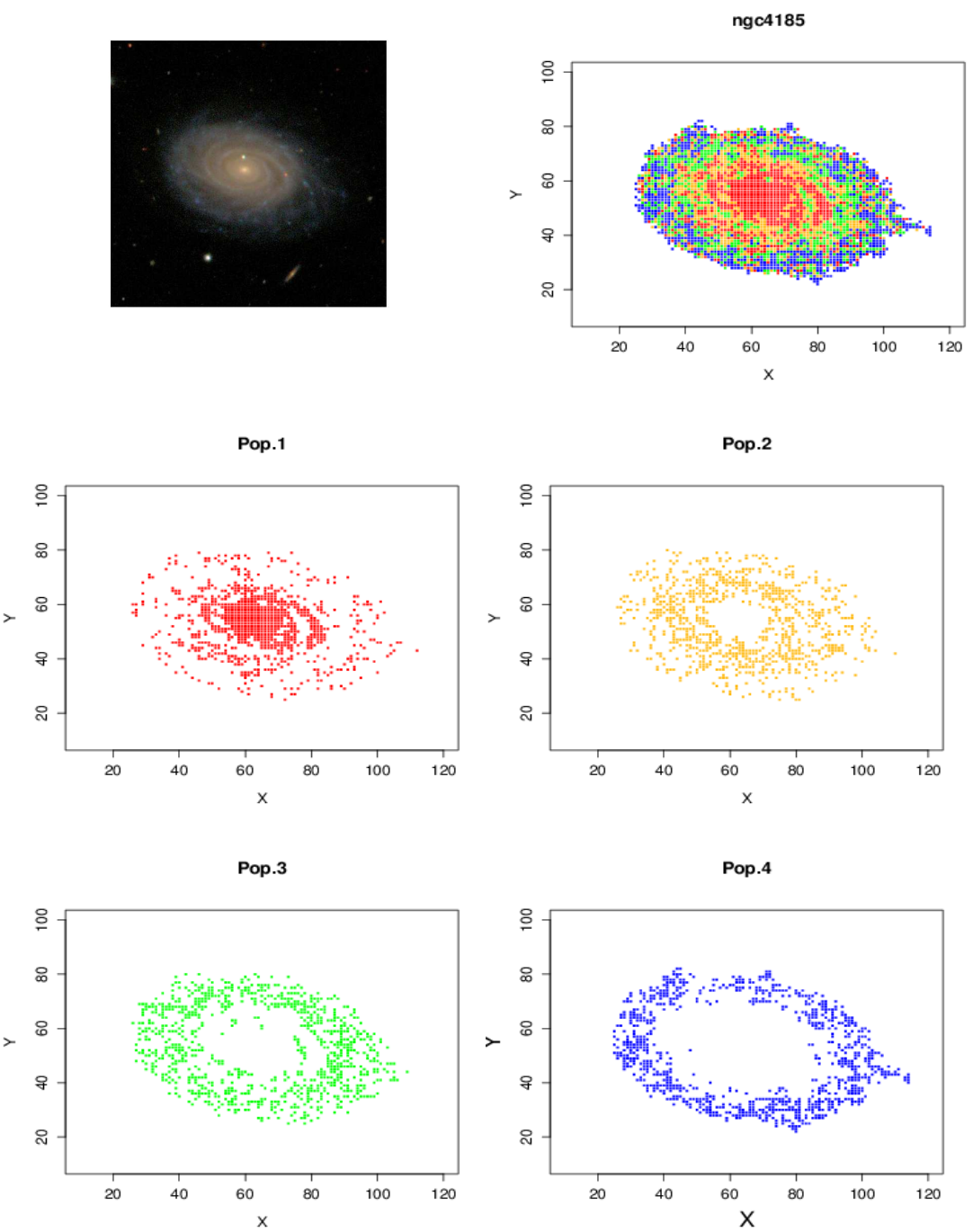

Figura 6.6: Distribuição espacial das populações estelares.

Na galáxia NGC4185, bem como nas demais galáxias espirais, a população mais vermelha está na região central e a população mais azul na borda, com as intermediárias distribuídas no entremeio da galáxia. Ainda, a galáxia NGC4185 exibe alguns sinais de 
braços espirais na população interna vermelha. Já a galáxia NGC2916 possui uma sobredensidade na população azul, podendo ser um efeito de projeção da imagem. Em suma, a distribuição das populações estelares nas galáxias espirais é consistente com uma evolução inside-out.
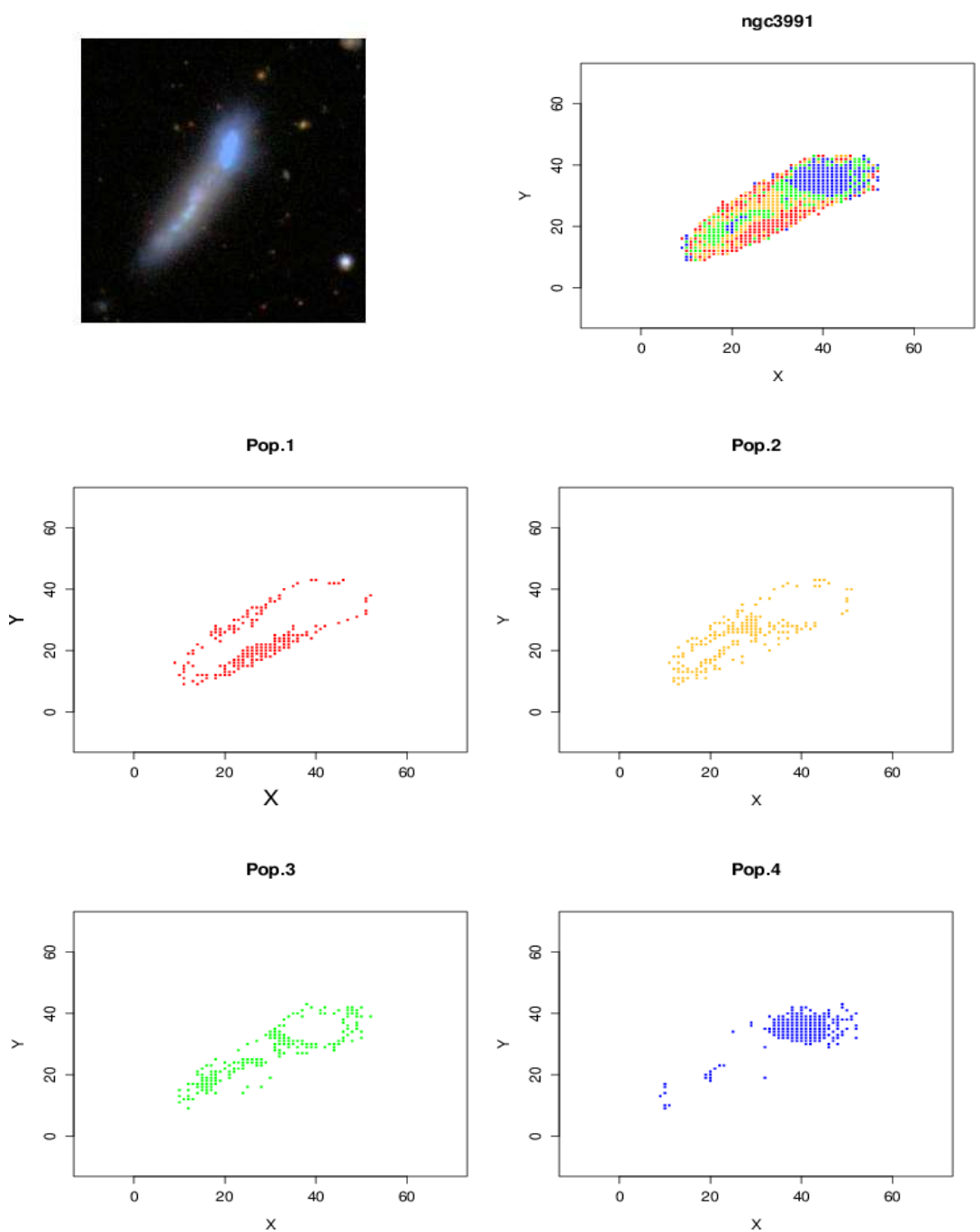

Figura 6.7: Distribuição espacial das populações estelares.

Dentre as galáxias espirais tardias, a galáxia NGC3991 mostra uma distribuição espacial de suas populações estelares bastante interessante. É notável a presença de uma região bastante azul na imagem óptica obtida do SDSS, a qual corresponde à população mais azul encontrada em nosso estudo. Nesse objeto, a população mais velha está majoritariamente na parte externa da cauda inferior da galáxia, enquanto que as populações intermediárias estão distribuídas dentro dessa cauda. É provável que esta galáxia tenha sofrido algum 
processo de fusão que ocasionou o desencadeamento de formação estelar recente na região superior, onde há a maior parte da população jovem. As demais espirais tardias tendem a ter uma distribuição não muito definida espacialmente, mas ainda assim é possível notar que, assim como no caso das espirais, as populações estelares tendem a se distribuir da mais vermelha na região central até as mais azuis na periferia.

\subsection{Cores e Raios Médios}

Seja o Raio de Petrosian $\mathfrak{R}_{P}$ o raio no qual a razão entre o brilho superficial anelar neste raio e o brilho superficial dentro dele é igual a 0.2 . Definindo um raio normalizado pelo Raio de Petrosian $\mathfrak{R}_{P}$ como sendo

$$
R_{N}=\frac{R_{i}}{\mathfrak{R}_{P}}
$$

calculamos o raio de cada píxel em relação ao centro óptico da galáxia, em cada uma das 4 populações, para cada galáxia da amostra.

A figura 6.8 exibe a cor $r$-i média de cada população em função do raio normalizado médio e, também, as populações em função do raio médio para as 3 galáxias exemplo, sendo que os gráficos para as demais galáxias estão apresentadas em A.3.

Nas galáxias elípticas, nota-se que as populações extremas, i.e., mais azuis e mais vermelhas, estão em regiões mais externas do que as populações P2 e P3, mas que ainda assim o raio mèdio da população azul P4 é externo a população P1. Como mencionado anteriormente, pode-se supor que tal característica possa ser atribuída a efeitos ambientais e de acresção. Na galáxia NGC6125, em específico, podemos notar que da população P2 a P4 temos um gradiente levemente negativo, mostrando que a formação estelar nessas regiões pode ter acontecido de dentro para fora, novamente evidenciando uma população que pode ter sido desencadeada por efeitos de minor merger central.

Já as galáxias espirais apresenta uma população estelar que se torna mais azul conforme se distancia do centro da galáxia. A distribuição das populações indicam um gradiente de cor negativo, ou seja, a cor $r-i$ média decresce com o raio ponderado médio, sendo esse gradiente de cor mais expressivo nas galáxias NGC7819 e NGC4185. Novamente, indícios de formação inside-out para as galáxias espirais.

$\mathrm{Na}$ análise da última sub-amostra de galáxias espirais tardias, encontramos um resultado semelhante ao caso das espirais, onde nota-se que a população P1 mais vermelha se 

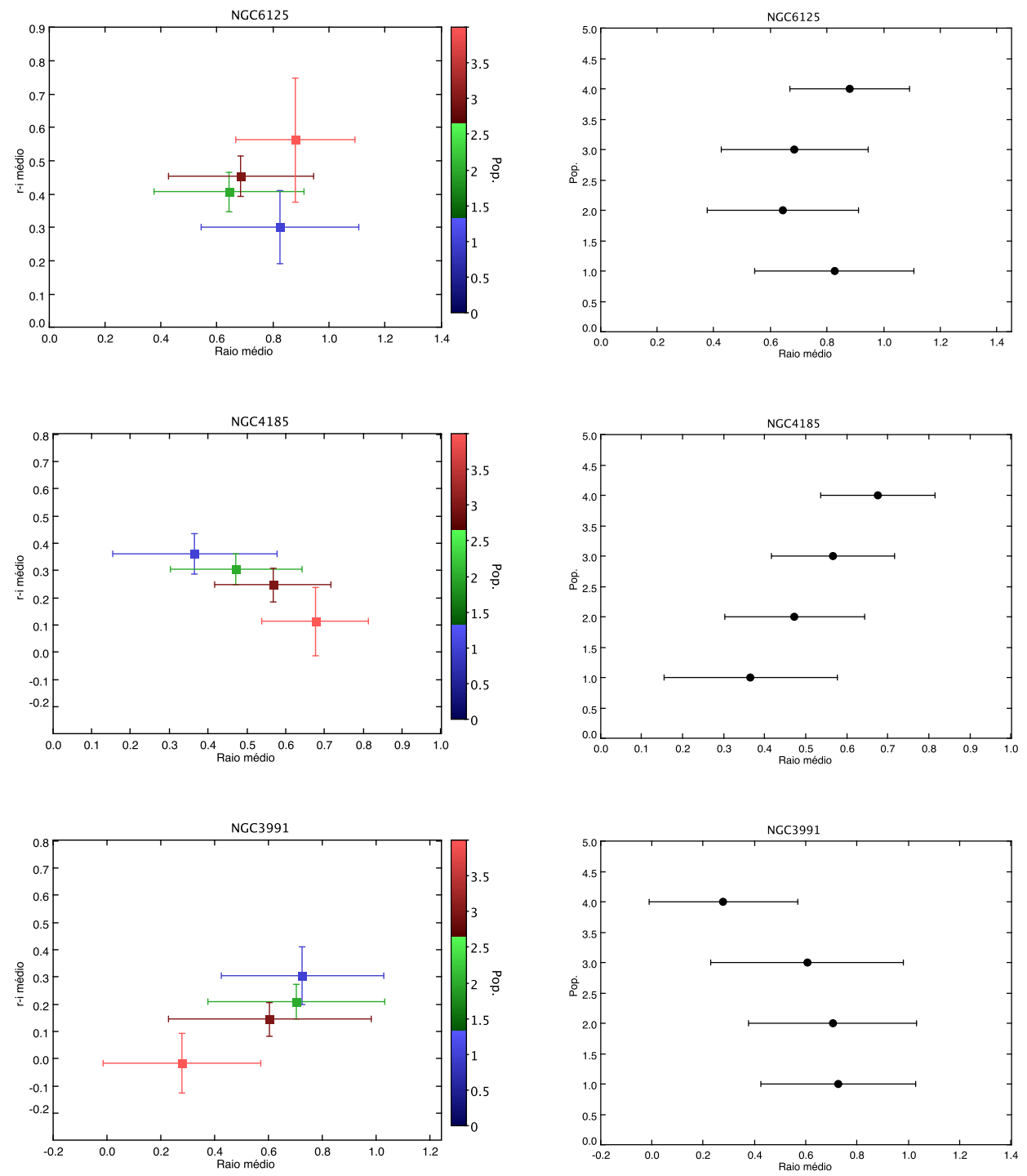

Figura 6.8: Raios médios normalizados e a cor g-r em cada população, para as galáxias NGC6125, NGC4185 e NGC3991. 
encontra na região central, e as populações vão se tornando mais azuis com o aumento do raio médio. Nesse grupo de galáxias, no entanto, é perceptível que as populações são mais misturadas e menos definidas que no caso da sub-amostra de galáxias espirais. Essa mistura de populações é uma característica que pode ser entendida como consequência da morfologia desses objetos, que tendem a ser mais irregulares. A galáxia NGC3991, como visto anteriormente, possui a população 4 mais azul numa região mais interna da galáxia, provavelmente devido a alguma interação recente.

\subsection{Coeficiente de Gini}

Utilizando o Coeficiente de Gini (CG), podemos obter informações a respeito da concentração da distribuição de contagens nos píxeis em cada população. Lembrando que $\mathrm{CG}=1$ corresponde a uma distribuição de contagens totalmente concentrada e $\mathrm{CG}=0$ uma população com distruibuição equitativa (com todos os píxeis contendo o mesmo valor de contagem), na tabela 6.1 são apresentados os Coeficientes de Gini de cada população. Ainda que todos os $\mathrm{CG}$ estejam bastante próximos de $\mathrm{CG}=0$, até mesmo pelo modo como foram determinadas as populações, é notável o fato de que o CG das populações diminuem da população 4 (mais azul) para a população 1 (mais vermelha), mostrando que as populações mais internas se tornam cada vez mais concentradas a medida que a população estelar se torna mais vermelha e velha.

Na figura 6.9 é apresentada relação entre os Coeficientes de Gini e as populações para as 3 galáxias exemplo e na tabela 6.1 estão os valores de CG para a amostra completa. Em todas as galáxias elípticas, observamos que o $\mathrm{CG}$ é muito próximo de $\mathrm{CG}=0$, indicando que as contagens em todas as populações são bem distribuidas. Além disso, vemos que o Coeficiente de Gini tende a aumentar da população P1 a P4, indicando que a distribuição de píxeis se torna mais igualitária na região central. Ainda, é interessante notar que nas galáxias NGC6173, NGC6125 e NGC6146 o CG da população mais externa P4 contraria essa tendência, sendo mais bem distribuida que a população P2 ou P3.

Nas galáxias espirais, observa-se que o CG também se mantêm crescente com as populações em todas as galáxias, mostrando que as populações estelares centrais são mais concentradas. A mesma tendência está presente nas galáxias espirais tardias, exceto na galáxia NGC3991 cujo CG é maior na região central e vai diminuindo até chegar na popu- 

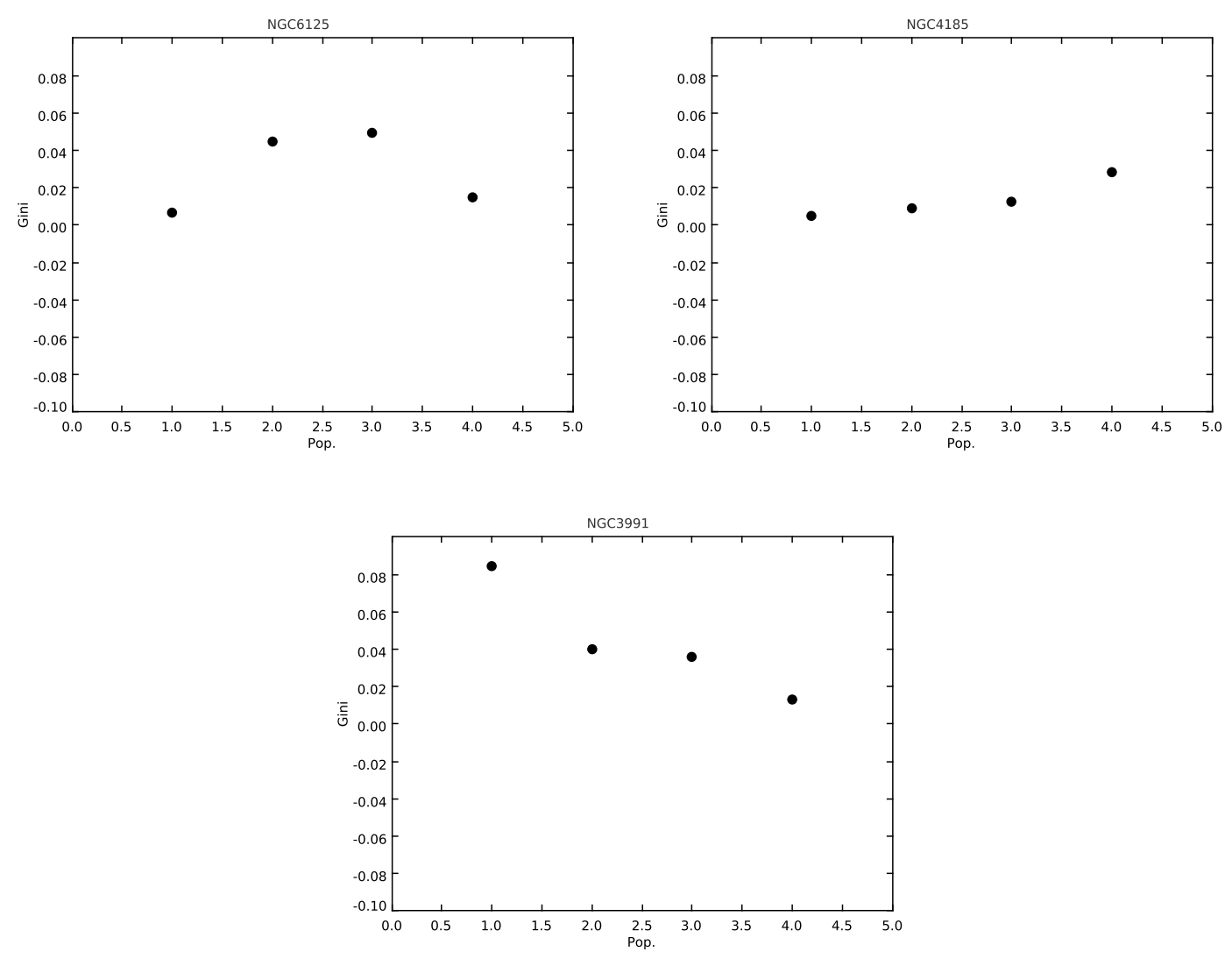

Figura 6.9: Coeficiente de Gini de cada população, para as galáxias NGC6125, NGC4185 e NGC3991. 
lação P4, indicando que nessa galáxia a distribuição dessa região é mais desigual que nas regiões periféricas. Ainda, é notável que a relação entre as populações e os Coeficiente de Gini para as galáxias UGC00312, UGC05358 e UGC10331 é quase constante.

Comparando os Coeficientes de Gini em função das populações para todas as galáxias (apêndice A.4) observamos que as galáxias espirais e espirais tardias tendem a ter o mesmo comportamento, excetuando-se a galáxia espiral tardia NGC3991, que como já mencionado pode ter suas características associadas ao fato de esse objeto ser um resultado de uma fusão de galáxias recente. Já as galáxias espirais tendem a ter o mesmo comportamento que as demais, ou seja, CG menores para as populações externas, mas apenas da população P2 a P4, sendo a população central P1 a que tem os píxeis menos bem distribuídos.

Tabela 6.1 - Coeficientes de Gini para as 4 populações estelares, para cada galáxia.

\begin{tabular}{lcccc}
\hline \hline Galáxia & Pop4 & Pop3 & Pop2 & Pop1 \\
\hline NGC6173 & 0.00313 & 0.0375 & 0.0376 & 0.0123 \\
NGC6125 & 0.0145 & 0.0488 & 0.0442 & 0.00623 \\
NGC6146 & 0.0293 & 0.0653 & 0.0288 & 0.00559 \\
NGC1349 & 0.0365 & 0.0205 & 0.0141 & 0.00525 \\
UGC05771 & 0.0669 & 0.0359 & 0.0124 & 0.00366 \\
NGC0776 & 0.0458 & 0.0176 & 0.0131 & 0.0113 \\
NGC2916 & 0.0567 & 0.0210 & 0.0158 & 0.0131 \\
NGC7819 & 0.0236 & 0.0129 & 0.00565 & 0.00368 \\
UGC03253 & 0.0498 & 0.0160 & 0.0112 & 0.00668 \\
NGC4185 & 0.0275 & 0.0122 & 0.00830 & 0.00465 \\
UGC00312 & 0.0274 & 0.0173 & 0.0174 & 0.0202 \\
UGC05358 & 0.00895 & 0.0138 & 0.00650 & 0.00513 \\
UGC09665 & 0.0573 & 0.0290 & 0.0192 & 0.00870 \\
UGC10331 & 0.0254 & 0.0233 & 0.0199 & 0.0163 \\
NGC3991 & 0.0125 & 0.0353 & 0.0396 & 0.0838 \\
& & & & \\
\hline
\end{tabular}

\subsection{Funcionais de Minkoswki}

Sendo a Característica de Euler-Poincaré (CEP) uma medida topológica da conectividade, queremos analisar se essa medida pode ser útil no estudo da morfologia e topologia das distribuições estelares, fornecendo informações a respeito da conectividade e porosidade dos píxeis de cada população. A figura 6.10 exibe a CEP de cada população das 
galáxias exemplo, tanto em valor absoluto (a esquerda) quanto normalizada (a direita). Ainda, na tabela 6.2 estão apresentados os valores absolutos e normalizados da CEP de todas as galáxias.

Analisando a Característica de Euler-Poincaré nas galáxias elípticas, não é observado nenhum comportamento padrão. Considerando que quanto maior a CEP menor a conetividade, aparentemente as populações das galáxias elípticas tendem a ser mais porosas do que as espirais e as espirais tardias. Nas galáxias espirais, excetuando-se a galáxia NGC7819, as população aparentam se tornar mais conexas na região central. Essa mesma tendência aparece nas espirais tardias, exceto na galáxia UGC00312. Essas tendências podem ser observadas nas figuras apresentadas no apêndice A.5.

Tabela 6.2 - Funcionais de Minkowski: Característica de Euler-Poincaré para cada população de cor.

\begin{tabular}{lcccccccc}
\hline \hline & \multicolumn{9}{c}{ CEP Absoluta } & & \multicolumn{5}{c}{ CEP Normalizada } \\
\hline & & & & & & & \\
Galáxia & Pop4 & Pop3 & Pop2 & Pop1 & Pop4 & Pop3 & Pop2 & Pop1 \\
\hline NGC6173 & 197 & 237 & 191 & 219 & 0.199 & 0.240 & 0.194 & 0.222 \\
NGC6125 & 161 & 196 & 156 & 170 & 0.188 & 0.229 & 0.182 & 0.199 \\
NGC6146 & 108 & 101 & 101 & 105 & 0.205 & 0.192 & 0.192 & 0.200 \\
NGC1349 & 86 & 91 & 92 & 74 & 0.212 & 0.225 & 0.227 & 0.183 \\
UGC05771 & 67 & 59 & 52 & 50 & 0.254 & 0.224 & 0.197 & 0.190 \\
NGC0776 & 91 & 98 & 95 & 55 & 0.173 & 0.187 & 0.187 & 0.105 \\
NGC2916 & 118 & 104 & 119 & 87 & 0.148 & 0.131 & 0.150 & 0.109 \\
NGC7819 & 55 & 77 & 77 & 40 & 0.153 & 0.215 & 0.214 & 0.111 \\
UGC03253 & 57 & 56 & 48 & 32 & 0.198 & 0.195 & 0.167 & 0.111 \\
NGC4185 & 153 & 156 & 145 & 81 & 0.178 & 0.182 & 0.168 & 0.094 \\
UGC00312 & 24 & 41 & 43 & 24 & 0.094 & 0.162 & 0.170 & 0.095 \\
UGC05358 & 41 & 33 & 36 & 27 & 0.235 & 0.189 & 0.206 & 0.156 \\
UGC09665 & 24 & 25 & 23 & 9 & 0.136 & 0.142 & 0.134 & 0.051 \\
UGC10331 & 28 & 31 & 25 & 22 & 0.169 & 0.189 & 0.152 & 0.134 \\
NGC3991 & 18 & 27 & 20 & 13 & 0.103 & 0.155 & 0.114 & 0.074 \\
& & & & & & & & \\
\hline & & & & & & & &
\end{tabular}



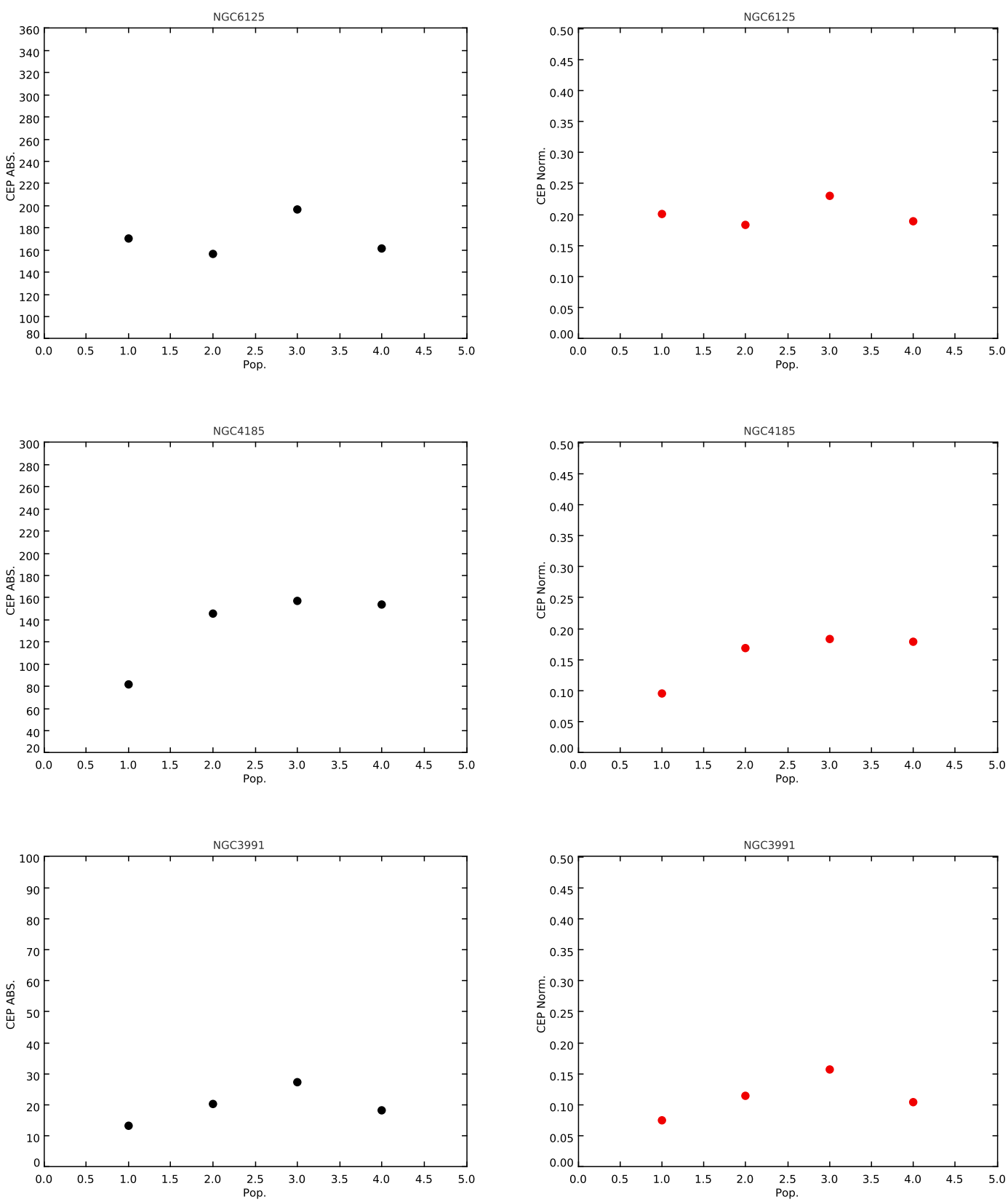

Figura 6.10: Característica de Euler-Poincaré de cada população, para as galáxias NGC6125, NGC4185 e NGC3991. A esquerda está CEP absoluta e a direita está a CEP normalizada. 
Capítulo 7

\section{Conclusões e Perspectivas}

Neste último capítulo, serão apresentadas as principais conclusões do trabalho (seção 7.1) e as perspectivas de aplicação em trabalhos futuros (seção 7.2).

\subsection{Conclusões}

Populações estelares são registros fósseis dos processos pelos quais as galáxias passaram durante sua formação e evolução. Utilizando uma abordagem píxel a píxel, analisamos uma pequena amostra de 15 galáxias de diferentes tipos morfológicos com um certo conjunto de estimadores estatísticos a fim de estudar a viabilidade de tal metodologia.

A análise PCA das magnitudes em cada píxel foi utilizada na classificação e determinação de populações estelares. A primeira componente principal (PC1) mostrou boa correlação com as cores em cada píxel, de modo que foi possível utilizá-la como um proxy para as populações estelares, através de sua ordenação e binagem em 4 populações. Dada as características fotométricas das imagens, a análise usou como base de estudos a cor $r-i$ dos píxeis.

Analisando espacialmente cada uma dessas populações para todas as galáxias, viu-se que as populações estelares tendem a se organizarem em regiões bem definidas no caso das galáxias espirais e espirais tardias, mas em regiões não tão definidas nas elípticas. Nas espirais e espirais tardias as populações mais vermelhas, e portanto mais velhas, tendem a ficar confinadas basicamente na região central das galáxias, com suas cores se tornando mais azuis à medida que atingem as regiões periféricas, sendo que no caso das espirais tardias a distribuição é mais assimétrica até mesmo pelo fato de esses objetos terem formatos mais peculiares. Essa constatação vai ao encontro do cenário inside-out que 
propõe que as galáxias evoluem de dentro para fora (Bell et al., 2004; Daddi et al., 2005; Muñoz-Mateos et al., 2007; Wang et al., 2011). Ainda, notou-se que em algumas galáxias espirais as características de braços ficaram um pouco mais perceptíveis, indicando que deve haver uma quantidade específica de populações estelares que maximize a capacidade de revelar detalhes acerca da distribuição espacial desses braços. No caso das elípticas, podemos perceber que há populações estelares mais jovens nas regiões intermediárias das galáxias. Considerando que, em princípio, as galáxias elípticas são objetos velhos que evoluem passivamente, a presença de populações mais jovens nas regiões centrais e intermediárias sugere que podem ter acontecido eventos ambientais ou ainda de minor mergers que podem ter fornecido gás e poeira necessários para o desencadeamento de uma novo surto de formação estelar, posterior ao surto de formação inicial. A distribuição de populações nas elípticas parece estar de acordo com o cenário hierárquico de fusão molhada, onde se espera que haja surtos intensos de formação estelar no centro das galáxias (Renzini. 2006; Di Matteo et al., 2009).

Corroborando com a distribuição espacial das populações estelares, a análise da cor $r$ - $i$ média e as populações em função dos raios normalizados médios realçou a questão da evolução inside-out das espirais e espirais tardias, além do cenário hierárquico de formação das elípticas. A análise do gradiente de cor nas galáxias elípticas mostrou que esse tipo de galáxia possui um gradiente positivo, com populações jovens não restritas a regiões externas, aspecto esse que pode estar conectado tanto ao fato de galáxias desse tipo terem cessado sua formação estelar há muito tempo, evoluindo passivamente, quanto aos efeitos das fusões molhadas que tendem a gerar surtos de formação estelar (Di Matteo et al., 2009). Dadas essas características do gradiente de cor, seria interessante analisar o meio no qual as galáxias se encontram, para estudar se há alguma possibilidade de ter sido desencadeado algum surto de formação mais recente próximo as regiões centrais (Toomre e Toomre, 1972; Renzini, 2006).

Já os gradientes de cor nas galáxias espirais e espirais tardias se mostraram negativos, ou seja, indicando que nesses objetos a evolução inside-out é bastante notável (Huang et al., 2013). Além disso, nota-se que a variação de cor nas galáxias espirais tardias são mais sutis, evidenciando que suas populações são bastantes homogêneas no sentido de que há formação estelar recente em toda a extensão da galáxia (Hunter, 1997; Cioni et al., 2000).

A análise da contagens nos píxeis das populações mostrou que todas as distribuições 
possuem Coeficiente de Gini deveras próximo de $\mathrm{CG}=0$, denotando que os valores das contagens das populações se distribuem de modo bastante igualitário. Comparando as populações umas com as outras, percebe-se que as contagens nos píxeis das populações centrais tendem a ser as mais bem distribuídas que as populações adjacentes. Essa característica se faz pertinente, uma vez que é na região central que há uma maior densidade de estrelas velhas que estão evoluindo passivamente (Katz, 1991; Arimoto, 1996; Kobayashi, 2004; Merlin e Chiosi, 2006). A única galáxia que contrariou tal tendência foi a galáxia NGC3991.

A análise da Característica de Euler-Poincaré (CEP) objetiva estudar a conectividade das populações estelares. Entendendo a CEP como uma medida da porosidade da distribuição da população, verificou-se que, ainda que não haja uma regularidade entre o valor da CEP e a população, a CEP das populações estelares das galáxias elípticas são maiores do que as galáxias espirais e espirais tardias, indicando que essas galáxias possuem populações estelares menos conexas e, por tanto, mais porosas.

Num primeiro momento, a análise da CEP das populações estelares não revelou muitas informações sobre as mesmas, entretanto, essa falta de tendência pode estar associada ao pequeno número de objetos utilizado no presente estudo. É possível que a análise de uma amostra estatisticamente significativa mostre que analisar a conectividade/porosidade das populações pode vir a ser ainda bastante útil.

Conforme visto no capítulo 6, a abordagem píxel a píxel das populações estelares nas galáxias se mostrou uma abordagem bastante frutífera, podendo trazer um novo olhar sobre a forma como entendemos a distribuição espacial das populações estelares dentro das galáxias e abrindo inúmeras possibilidades de estudo.

\subsection{Perspectivas}

Uma vez que esta nova abordagem se mostrou promissora, pretende-se ampliar esta análise para uma amostra de dados maior, tais como as fornecidas por grandes surveys como o SDSS, J-PAS ou ALHAMBRA. A perspectiva é que tal metodologia possibilite um estudo ainda mais aprofundado de como a distribuição espacial e as medidas das principais características das populações estelares influenciam na formação e evolução das galáxias.

Um próximo passo natural desse projeto é a automatização das análises, de forma que 
seja possível aplicar tal approach em um grande número de dados, podendo assim alcançar resultados estatisticamente relevantes, sendo capaz inclusive de mostrar tendências ainda inimagináveis.

Por último, pretende-se ampliar esta técnica para que seja possível utilizar dados com informações espectrais (IFU) ou em um número maior de bandas, tais como os dados que serão ofertados pelos projetos J-PAS (56 bandas) e ALHAMBRA (20 bandas). 


\section{Referências Bibliográficas}

Abazajian K. N., Adelman-McCarthy J. K., Agüeros M. A., Allam S. S., Allende Prieto C., An D., Anderson K. S. J., Anderson S. F., Annis J., Bahcall N. A., et al. The Seventh Data Release of the Sloan Digital Sky Survey, ApJS, 2009, vol. 182, p. 543

Abraham R. G., van den Bergh S., Nair P., P. P., A New Approach to Galaxy Morphology. I. Analysis of the Sloan Digital Sky Survey Early Data Release, ApJ, 2003, vol. 588, p. 218

Aihara H., Allende Prieto C., An D., Anderson S. F., Aubourg É., Balbinot E., Beers T. C., Berlind A. A., Bickerton S. J., Bizyaev D., The Eighth Data Release of the Sloan Digital Sky Survey: First Data from SDSS-III, ApJS, 2011, vol. 193, p. 29

Alongi M., Bertelli G., Bressan A., Chiosi C., Fagotto F., Greggio L., Nasi E., Evolutionary sequences of stellar models with semiconvection and convective overshoot. I - Z $=0.008$, A\&AS, 1993, vol. 97, p. 851

Andreon S., Gargiulo G., Longo G., Tagliaferri R., Capuano N., Wide field imaging I. Applications of neural networks to object detection and star/galaxy classification, MNRAS, 2000, vol. 319, p. 700

Arimoto N., Stellar Population Synthesis Models for Elliptical Galaxies. In From Stars to Galaxies: the Impact of Stellar Physics on Galaxy Evolution, vol. 98 of Astronomical Society of the Pacific Conference Series, 1996, p. 287

Baade W., The Resolution of Messier 32, NGC 205, and the Central Region of the Andromeda Nebula., ApJ, 1944, vol. 100, p. 137 
Baldry I. K., Glazebrook K., Brinkmann J., Ivezić Ž., Lupton R. H., Nichol R. C., Szalay A. S., Quantifying the Bimodal Color-Magnitude Distribution of Galaxies, ApJ, 2004, vol. 600 , p. 681

Barker M. K., Sarajedini A., Geisler D., Harding P., Schommer R., The Stellar Populations in the Outer Regions of M33. III. Star Formation History, AJ, 2007, vol. 133, p. 1138

Belcheva M. K., Livanou E., Kontizas M., Nikolov G. B., Kontizas E., Spatial distribution of stellar populations in the Magellanic Clouds: implementation to Gaia, A\&A, 2011, vol. 527, p. A31

Bell E. F., Wolf C., Meisenheimer K., Rix H.-W., Borch A., Dye S., Kleinheinrich M., Wisotzki L., McIntosh D. H., Nearly 5000 Distant Early-Type Galaxies in COMBO-17: A Red Sequence and Its Evolution since z 1, ApJ, 2004, vol. 608, p. 752

Bruzual G., Charlot S., Stellar population synthesis at the resolution of 2003, MNRAS, 2003, vol. 344, p. 1000

Calzetti D., Star Formation Rate Indicators, ArXiv e-prints, 2012

Carruba V., An analysis of the Hygiea asteroid family orbital region, MNRAS, 2013

Chabrier G., Galactic Stellar and Substellar Initial Mass Function, PASP, 2003, vol. 115, p. 763

Cid Fernandes R., Asari N. V., Sodré L., Stasińska G., Mateus A., Torres-Papaqui J. P., Schoenell W., Uncovering the chemical enrichment and mass-assembly histories of starforming galaxies, MNRAS, 2007, vol. 375, p. L16

Cid Fernandes R., Mateus A., Sodré L., Stasińska G., Gomes J. M., Semi-empirical analysis of Sloan Digital Sky Survey galaxies - I. Spectral synthesis method, MNRAS, 2005, vol. 358 , p. 363

Cioni M.-R. L., Habing H. J., Israel F. P., The morphology of the Magellanic Clouds revealed by stars of different age: results from the DENIS survey, A\&A, 2000, vol. 358, p. L9

Colless M., First results from the 2dF Galaxy Redshift Survey, Royal Society of London Philosophical Transactions Series A, 1999, vol. 357, p. 105 
Colless M., Boyle B., Redshift Surveys with 2DF, Highlights of Astronomy, 1998, vol. 11, p. 473

Connolly A. J., Szalay A. S., Bershady M. A., Kinney A. L., Calzetti D., Spectral Classification of Galaxies: an Orthogonal Approach, AJ, 1995, vol. 110, p. 1071

Costa-Duarte M. V., Sodré Jr. L., Durret F., Morphological properties of superclusters of galaxies, MNRAS, 2011, vol. 411, p. 1716

Daddi E., Renzini A., Pirzkal N., Cimatti A., Malhotra S., Stiavelli M., Xu C., Pasquali A., Rhoads J. E., Brusa M., di Serego Alighieri S., Ferguson H. C., Koekemoer A. M., Moustakas L. A., Panagia N., Windhorst R. A., Passively Evolving Early-Type Galaxies at $1.4<\sim z<\sim 2.5$ in the Hubble Ultra Deep Field, ApJ, 2005, vol. 626, p. 680

de Vaucouleurs G., de Vaucouleurs A., Corwin H. G., Buta R. J., Paturel G., Fouque P., Third Reference Cat. of Bright Galaxies (RC3) (de Vaucouleurs+ 1991), VizieR Online Data Catalog, 1995, vol. 7155, p. 0

de Vaucouleurs G., de Vaucouleurs A., Corwin Jr. H. G., Second reference catalogue of bright galaxies. Containing information on 4,364 galaxies with references to papers published between 1964 and 1975., 1976

de Vaucouleurs G. H., de Vaucouleurs A., Shapley H., Reference catalogue of bright galaxies, 1964

Di Matteo P., Pipino A., Lehnert M. D., Combes F., Semelin B., On the survival of metallicity gradients to major dry-mergers, A\&A, 2009, vol. 499, p. 427

Díaz E., Zandivarez A., Merchán M. E., Muriel H., Two Degree Field Galaxy Redshift Survey and Sloan Digital Sky Survey Galaxy Group Density Profiles, ApJ, 2005, vol. 629, p. 158

Dottori H., Bica E., Claria J. J., Puerari I., Spatial Distributions of Young Large Magellanic Cloud Clusters as Tracers of a Bar Perturbation, ApJ, 1996, vol. 461, p. 742

Eggen O. J., Lynden-Bell D., Sandage A. R., Evidence from the motions of old stars that the Galaxy collapsed., ApJ, 1962, vol. 136, p. 748 
Faber S. M., Quadratic programming applied to the problem of galaxy population synthesis., A\&A, 1972, vol. 20, p. 361

Fall S. M., Efstathiou G., Formation and rotation of disc galaxies with haloes, MNRAS, 1980, vol. 193, p. 189

Ferguson A. M. N., Clarke C. J., The evolution of stellar exponential discs, MNRAS, 2001, vol. 325 , p. 781

Folkes S. R., Lahav O., Maddox S. J., An artificial neural network approach to the classification of galaxy spectra., MNRAS, 1996, vol. 283, p. 651

Freeman K., Bland-Hawthorn J., The New Galaxy: Signatures of Its Formation, ARA\&A, 2002, vol. 40, p. 487

García Benito R., Pérez Jiménez E., González Delgado R. R. C. F., Sánchez S. F., Amorim A. L., Califa Collaboration The mass growth in galaxies: CALIFA's perspective. In Highlights of Spanish Astrophysics VII , 2013, p. 218

Gargiulo A., Saracco P., Longhetti M., La Barbera F., Tamburri S., Spatially resolved colours and stellar population properties in early-type galaxies at $\mathrm{z} \sim 1.5$, MNRAS, 2012, vol. 425, p. 2698

Gini C., Variabilità e mutabilità, reprinted in Memorie di Metodologia Statistica, 1912

Girardi L., Bressan A., Chiosi C., Bertelli G., Nasi E., Evolutionary sequences of stellar models with new radiative opacities. VI. Z=0.0001., A\&AS, 1996, vol. 117, p. 113

Glasser G. J., Variance formulas for the mean difference and coefficient of concentration, Journal of the American Statistical Association, 1962, vol. 57

Gonidakis I., Livanou E., Kontizas E., Klein U., Kontizas M., Belcheva M., Tsalmantza P., Karampelas A., Structure of the SMC. Stellar component distribution from 2MASS data, A\&A, 2009, vol. 496, p. 375

González Delgado R., Pérez E., Cid Fernandes R., García-Benito R., de Amorim A. L., Sánchez S. F., Husemann B., López Fernández R., Cortijo-Ferrero CALIFA survey: The spatially resolved star formation history of massive galaxies, ArXiv e-prints, 2013 
Harnois-Déraps J., Pen U.-L., Non-Gaussian error bars in galaxy surveys - II, MNRAS, 2013

Helou G., Madore B. F., Schmitz M., Wu X., Corwin Jr. H. G., Lague C., Bennett J., Sun H., The NASA/IPAC Extragalactic Database.. In Information On-Line Data in Astronomy, vol. 203 of Astrophysics and Space Science Library, 1995, p. 95

Hikage C., Schmalzing J., Buchert T., Suto Y., Kayo I., Taruya A., Vogeley M. S., Hoyle F., Gott III J. R., Brinkmann J., Minkowski Functionals of SDSS Galaxies I : Analysis of Excursion Sets, PASJ, 2003, vol. 55, p. 911

Hopkins P. F., Bundy K., Murray N., Quataert E., Lauer T. R., Ma C.-P., Compact highredshift galaxies are the cores of the most massive present-day spheroids, MNRAS, 2009, vol. 398 , p. 898

Hotelling H., Analysis of a Complex of Statistical Variables Into Principal Components, Journal of Educational Psychology, 1933

Huang M.-L., Kauffmann G., Chen Y.-M., Moran S. M., Heckman T. M., Davé R., Johansson J., Spatially resolved star formation histories of nearby galaxies: evidence for episodic star formation in discs, MNRAS, 2013, vol. 431, p. 2622

Hubble E., A Relation between Distance and Radial Velocity among Extra-Galactic Nebulae, Proceedings of the National Academy of Science, 1929, vol. 15, p. 168

Hubble E. P., Photographic investigations of faint nebulae, Publications of the Yerkes Observatory, 1920, vol. 4, p. 2

Hubble E. P., A general study of diffuse galactic nebulae., ApJ, 1922, vol. 56, p. 162

Hubble E. P., Extragalactic nebulae., ApJ, 1926, vol. 64, p. 321

Huchra J., Davis M., Latham D., Tonry J., A survey of galaxy redshifts. IV - The data, ApJS, 1983, vol. 52, p. 89

Hunter D., Star Formation in Irregular Galaxies: A Review of Several Key Questions, PASP, 1997, vol. 109, p. 937 
Husemann B., Jahnke K., Sánchez S. F., Barrado D., Bekeraite S., Bomans D. J., CastilloMorales A., Catalán-Torrecilla C., Cid Fernandes R., Falcón-Barroso J., García-Benito R., González Delgado R., CALIFA, the Calar Alto Legacy Integral Field Area survey. II. First public data release, A\&A, 2013, vol. 549, p. A87

Ivezic Z., Tyson J. A., Axelrod T., Burke D., Claver C. F., Cook K. H., Kahn S. M., Lupton R. H., Monet D. G., Pinto P. A., Strauss M. A., Stubbs C. W., Jones L., Saha A., Scranton R., Smith C., LSST Collaboration LSST: From Science Drivers To Reference Design And Anticipated Data Products. In American Astronomical Society Meeting Abstracts 213 , vol. 41 of Bulletin of the American Astronomical Society, 2009, p. 460.03

Katz N., Dissipationless collapse in an expanding universe, ApJ, 1991, vol. 368, p. 325

Kauffmann G., Heckman T. M., White S. D. M., Charlot S., Tremonti C., Peng E. W., Seibert M., Brinkmann J., Nichol R. C., SubbaRao M., York D., The dependence of star formation history and internal structure on stellar mass for $10^{5}$ low-redshift galaxies, MNRAS, 2003, vol. 341, p. 54

Kelz A., Verheijen M. A. W., Roth M. M., Bauer S. M., Becker T., Paschke J., Popow E., Sánchez S. F., Laux U., PMAS: The Potsdam Multi-Aperture Spectrophotometer. II. The Wide Integral Field Unit PPak, PASP, 2006, vol. 118, p. 129

Kobayashi C., GRAPE-SPH chemodynamical simulation of elliptical galaxies - I. Evolution of metallicity gradients, MNRAS, 2004, vol. 347, p. 740

Kong X., Charlot S., Brinchmann J., Fall S. M., Star formation history and dust content of galaxies drawn from ultraviolet surveys, MNRAS, 2004, vol. 349, p. 769

Kučinskas A., Bridžius A., Vansevičius V., Globular Clusters in the Large Magellanic Cloud: An Impact from GAIA Photometry, Ap\&SS, 2002a, vol. 280, p. 159

Kučinskas A., Bridžius A., Vansevičius V., Stellar Populations in the Large Magellanic Cloud: The Impact from GAIA. In EAS Publications Series, vol. 2 of EAS Publications Series, 2002b, p. 383 
Lara-López M. A., López-Sánchez Á. R., Hopkins A. M., On the Three-dimensional Structure of the Mass, Metallicity, and Star Formation Rate Space for Star-forming Galaxies, ApJ, 2013, vol. 764, p. 178

Larson R. B., Tinsley B. M., Photometric Properties of Model Spherical Galaxies, ApJ, 1974, vol. 192, p. 293

Le Borgne J.-F., Bruzual G., Pelló R., Lançon A., Rocca-Volmerange B., Sanahuja B., Schaerer D., Soubiran C., Vílchez-Gómez R., STELIB: A library of stellar spectra at R 2000, A\&A, 2003, vol. 402, p. 433

Leitherer C., Schaerer D., Goldader J. D., González Delgado R. M., Robert C., Kune D. F., de Mello D. F., Devost D., Heckman T. M., Starburst99: Synthesis Models for Galaxies with Active Star Formation, ApJS, 1999, vol. 123, p. 3

Lin D. N. C., Pringle J. E., The formation of the exponential disk in spiral galaxies, ApJ, 1987, vol. 320, p. L87

Maragoudaki F., Kontizas M., Kontizas E., Dapergolas A., Morgan D. H., The LMC stellar complexes in luminosity slices. Star formation indicators, A\&A, 1998, vol. 338, p. L29

Maragoudaki F., Kontizas M., Morgan D. H., Kontizas E., Dapergolas A., Livanou E., The recent structural evolution of the SMC, A\&A, 2001, vol. 379, p. 864

Marino R. A., Gil de Paz A., Sánchez S. F., Castillo-Morales A., CALIFA Team Studying nearby disk galaxies with the CALIFA survey., Memorie della Societa Astronomica Italiana Supplementi, 2012, vol. 19, p. 290

Marzke R., McCarthy P. J., Persson E., Oemler A., Dressler A., Yan M. B. L., Carlberg R., Abraham R., Ellis R., Firth A., Mackay C., McMahon R. G., The Las Campanas Infrared Survey. In Photometric Redshifts and the Detection of High Redshift Galaxies , vol. 191 of Astronomical Society of the Pacific Conference Series, 1999, p. 148

Mateus A., Sodré L., Cid Fernandes R., Stasińska G., Semi-empirical analysis of Sloan Digital Sky Survey galaxies - IV. A nature via nurture scenario for galaxy evolution, MNRAS, 2007, vol. 374, p. 1457 
Mateus A., Sodré L., Cid Fernandes R., Stasińska G., Schoenell W., Gomes J. M., Semiempirical analysis of Sloan Digital Sky Survey galaxies - II. The bimodality of the galaxy population revisited, MNRAS, 2006, vol. 370, p. 721

McGurk R. C., Kimball A. E., Ivezić Ž., Principal Component Analysis of Sloan Digital Sky Survey Stellar Spectra, AJ, 2010, vol. 139, p. 1261

Mecke K. R., , Munich, 1993, Tese de Doutorado

Mecke K. R., Morphological characterization of patterns in reaction-diffusion systems, Phys. Rev. E, 1996, vol. 53, p. 4794

Merlin E., Chiosi C., Formation and evolution of early-type galaxies. II. Models with quasi-cosmological initial conditions, A\&A, 2006, vol. 457, p. 437

Morgan W. W., The Integrated Spectral Types of Globular Clusters, PASP, 1956, vol. 68, p. 509

Muñoz Bermejo J., Asensio Ramos A., Allende Prieto C., A PCA approach to stellar effective temperatures, ArXiv e-prints, 2013

Muñoz-Mateos J. C., Gil de Paz A., Boissier S., Zamorano J., Jarrett T., Gallego J., Madore B. F., Specific Star Formation Rate Profiles in Nearby Spiral Galaxies: Quantifying the Inside-Out Formation of Disks, ApJ, 2007, vol. 658, p. 1006

Murtagh F., Heck A., Multivariate Data Analysis 2 edn. Springer, 1987

Naab T., Ostriker J. P., A simple model for the evolution of disc galaxies: the Milky Way, MNRAS, 2006, vol. 366, p. 899

Nikolaev S., Weinberg M. D., Stellar Populations in the Large Magellanic Cloud from 2MASS, ApJ, 2000, vol. 542, p. 804

Ownsworth J. R., Conselice C. J., Mortlock A., Hartley W. G., Buitrago F., Evolution of massive galaxy structural properties and sizes via star formation in the GOODS NICMOS Survey, MNRAS, 2012, vol. 426, p. 764

Pearson K., On lines and planes of closest fit to systems of points in space, Philosophical Magazine, 1901 
Pérez E., Cid Fernandes R., González Delgado R. M., García-Benito R., Sánchez S. F., Husemann B., Mast D., Rodón J. R., Kupko D., Backsmann N., de Amorim A. L., van de Ven G., Walcher J., The Evolution of Galaxies Resolved in Space and Time: A View of Inside-out Growth from the CALIFA Survey, ApJ, 2013, vol. 764, p. L1

Rehse S., Characterization of the dynamics of block copolymer microdomains with local morphological measures, Phys. Rev. E, 2008

Renzini A., Stellar Population Diagnostics of Elliptical Galaxy Formation, ARA\&A, 2006, vol. 44 , p. 141

Rosenfeld A., Kak C., Digital Picture Processing, Academic, 1976

Roth M. M., Kelz A., Fechner T., Hahn T., Bauer S.-M., Becker T., Böhm P., Christensen L., Dionies F., Paschke J., Popow E., Wolter D., Schmoll J., Laux U., Altmann W., PMAS: The Potsdam Multi-Aperture Spectrophotometer. I. Design, Manufacture, and Performance, PASP, 2005, vol. 117, p. 620

Ruiz-Lara T., Florido E., Pérez I., Sánchez-Blázquez P., Falcón-Barroso J., Cacho R., Stellar populations in the outskirts of spiral galaxies using IFU CALIFA data. In Highlights of Spanish Astrophysics VII , 2013, p. 367

Sánchez S. F., Kennicutt R. C., Gil de Paz A., van de Ven G., Vílchez J. M., Wisotzki L., Walcher C. J., Mast D., Aguerri J. A. L., Albiol-Pérez S., Alonso-Herrero A., Alves J., Colaborations. C., CALIFA, the Calar Alto Legacy Integral Field Area survey. I. Survey presentation, A\&A, 2012, vol. 538, p. A8

Sánchez S. F., Kennicutt R. C., Gil de Paz A., van den Ven G., Vilchez J. M., Wisotzki L., Marino R. A., Mármol-Queraltó E., Mast D., Viironen K., CALIFA, the Calar Alto Legacy Integral Field Area survey: early report. In Highlights of Spanish Astrophysics VI , 2011, p. 339

Sánchez S. F., Rosales-Ortega F. F., Jungwiert B., Iglesias-Páramo J., Vílchez J. M., Marino R. A., Walcher C. J., Husemann B., Mast D., Monreal-Ibero A., Cid Fernandes R., Pérez E., Califa Collaboration Mass-metallicity relation explored with CALIFA. I. Is there a dependence on the star-formation rate?, A\&A, 2013, vol. 554, p. A58 
Sandage A., Tammann G. A., A revised Shapley-Ames Catalog of bright galaxies, 1980

Santalo L. A., Integral Geometry and Geometric Probability, Addison-Wesley, Reading, MA, 1976

Serra J., Image Analysis and Mathematical Morphology, Academic, 1982, vol. 1 and 2

Shapley H., Ames A., A survey of the external galxies brighter than the thirteenth magnitude, Annals of Harvard College Observatory, 1932, vol. 88, p. 41

Shectman S. A., Landy S. D., Oemler A., Tucker D. L., Lin H., Kirshner R. P., Schechter P. L., The Las Campanas Redshift Survey, ApJ, 1996, vol. 470, p. 172

Sodré L., Cuevas H., Global regularities in integrated galaxy spectra, MNRAS, 1997, vol. 287, p. 137

Sodré Jr. L., Cosmology with large galaxy redshift surveys. In American Institute of Physics Conference Series, vol. 1471 of American Institute of Physics Conference Series, 2012a, p. 22

Sodré Jr. L., Optical Galaxy redshift surveys. In SF2A-2012: Proceedings of the Annual meeting of the French Society of Astronomy and Astrophysics , 2012b, p. 481

Sodré Jr. L., Stasińska G., The emission line sequence of normal spiral galaxies, A\&A, 1999, vol. 345, p. 391

Spinrad H., Taylor B. J., The Stellar Content of the Nuclei of Nearby Galaxies. II. a Note on NGC 4594, ApJ, 1972, vol. 171, p. 397

Spite M., GAIA and the Stellar Populations in the Magellanic Clouds. In EAS Publications Series, vol. 2 of EAS Publications Series, 2002, p. 287

Strateva I., Ivezić Ž., Knapp G. R., Narayanan V. K., Strauss M. A., Gunn J. E., Lupton R. H., Schlegel D., Bahcall N. A., Brinkmann J., Brunner R. J., Budavári T., Color Separation of Galaxy Types in the Sloan Digital Sky Survey Imaging Data, AJ, 2001, vol. 122 , p. 1861

Tinsley B. M., Evolution of the Stars and Gas in Galaxies, Fund. Cosmic Phys., 1980, vol. 5 , p. 287 
Tinsley B. M., Gunn J. E., Evolutionary synthesis of the stellar population in elliptical galaxies. I - Ingredients, broad-band colors, and infrared features, ApJ, 1976, vol. 203, p. 52

Toomre A., Toomre J., Galactic Bridges and Tails, ApJ, 1972, vol. 178, p. 623

Trac H., Mitsouras D., Hickson P., Brandenberger R., Topology of the Las Campanas Redshift Survey, MNRAS, 2002, vol. 330, p. 531

Vale Asari N., Stasińska G., Cid Fernandes R., Gomes J. M., Schlickmann M., Mateus A., Schoenell W., The evolution of the mass-metallicity relation in SDSS galaxies uncovered by astropaleontology, MNRAS, 2009, vol. 396, p. L71

van der Marel R. P., Magellanic Cloud Structure from Near-Infrared Surveys. II. Star Count Maps and the Intrinsic Elongation of the Large Magellanic Cloud, AJ, 2001, vol. 122 , p. 1827

van der Marel R. P., Cioni M.-R. L., Magellanic Cloud Structure from Near-Infrared Surveys. I. The Viewing Angles of the Large Magellanic Cloud, AJ, 2001, vol. 122, p. 1807

Verheijen M. A. W., Bershady M. A., Andersen D. R., Swaters R. A., Westfall K., Kelz A., Roth M. M., The Disk Mass project; science case for a new PMAS IFU module, Astronomische Nachrichten, 2004, vol. 325, p. 151

Wang J., Kauffmann G., Overzier R., Catinella B., Schiminovich D., Heckman T. M., Moran S. M., Haynes M. P., Giovanelli R., Kong X., The GALEX Arecibo SDSS survey - III. Evidence for the inside-out formation of Galactic discs, MNRAS, 2011, vol. 412, p. 1081

Wood D. B., Multicolor Photoelectric Photometry of Galaxies, ApJ, 1966, vol. 145, p. 36

Wuyts S., Cox T. J., Hayward C. C., Franx M., Hernquist L., Hopkins P. F., Jonsson P., van Dokkum P. G., On Sizes, Kinematics, M/L Gradients, and Light Profiles of Massive Compact Galaxies at $\mathrm{z} \sim 2$, ApJ, 2010, vol. 722, p. 1666

Yip C. W., Connolly A. J., Vanden Berk D. E., Ma Z., Frieman J. A., SubbaRao M., Szalay A. S., Richards G. T., Hall P. B., Schneider D. P., Hopkins A. M., Trump J., Brinkmann 
J., Spectral Classification of Quasars in the Sloan Digital Sky Survey: Eigenspectra, Redshift, and Luminosity Effects, AJ, 2004, vol. 128, p. 2603

Zhao G.-B., Pogosian L., Silvestri A., Zylberberg J., Cosmological Tests of General Relativity with Future Tomographic Surveys, Physical Review Letters, 2009, vol. 103, p. 241301 
Apêndice 



\section{Apêndice A}

\section{Apêndice - Resultados}

\section{A.1 PCA e Populações Estelares Píxel a Píxel}

A seguir são apresentadas as correlações entre a primeira componente principal e as cores $u-g, g-r, r-i$ e $i-z$ para as galáxias elípticas A.1.1, espirais A.1.2 e espirais tardias A.1.3. Dada a arbitrariedade da orientação da PCA, convencionou-se que quanto maior a primeira componente principal, maior a cor dos píxeis.

\section{A.1.1 Galáxias Elípticas}

PCA em função das cores $u-g, g-r, r-i$ e $i$-z para as 4 populações estelares, nas galáxias da subamostra de galáxias elípticas. 

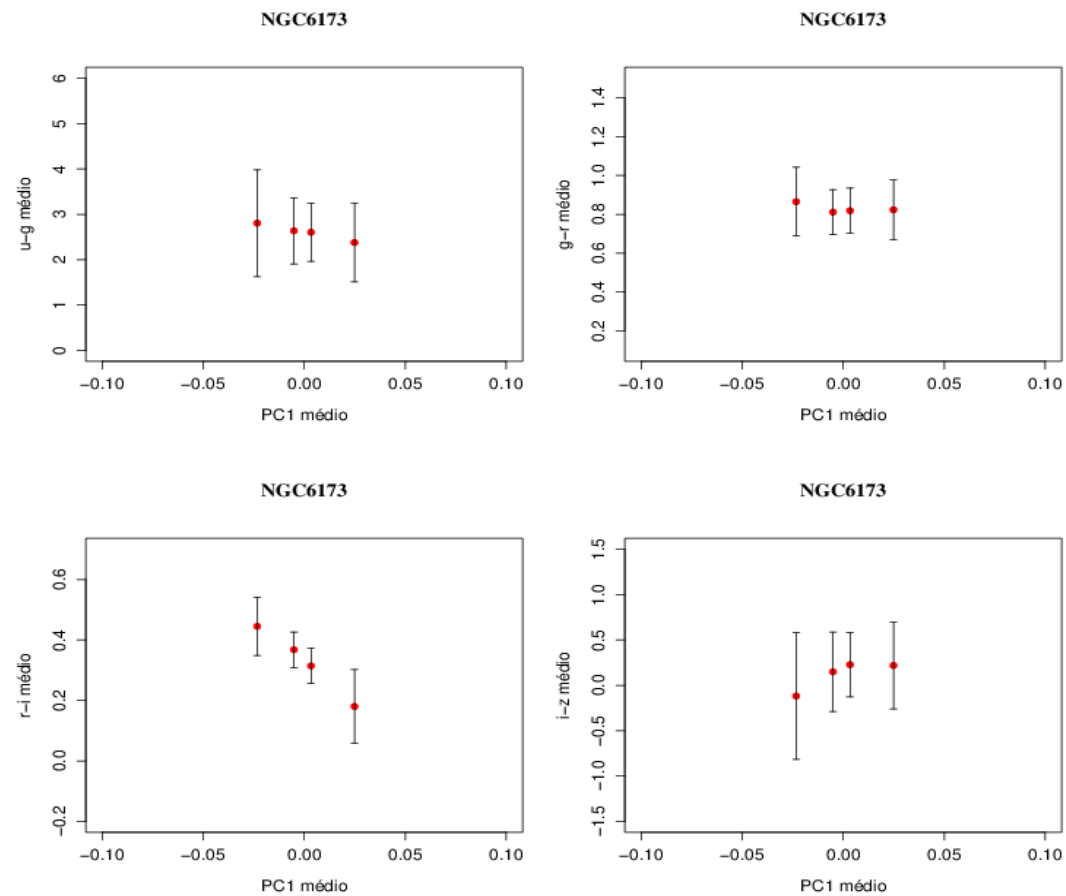

Figura A.1: Análise PCA nos píxeis: As cores $u-g, g-r, r-i$ e $i-z$ em função da $1^{a}$ componente principal, para NGC6173.
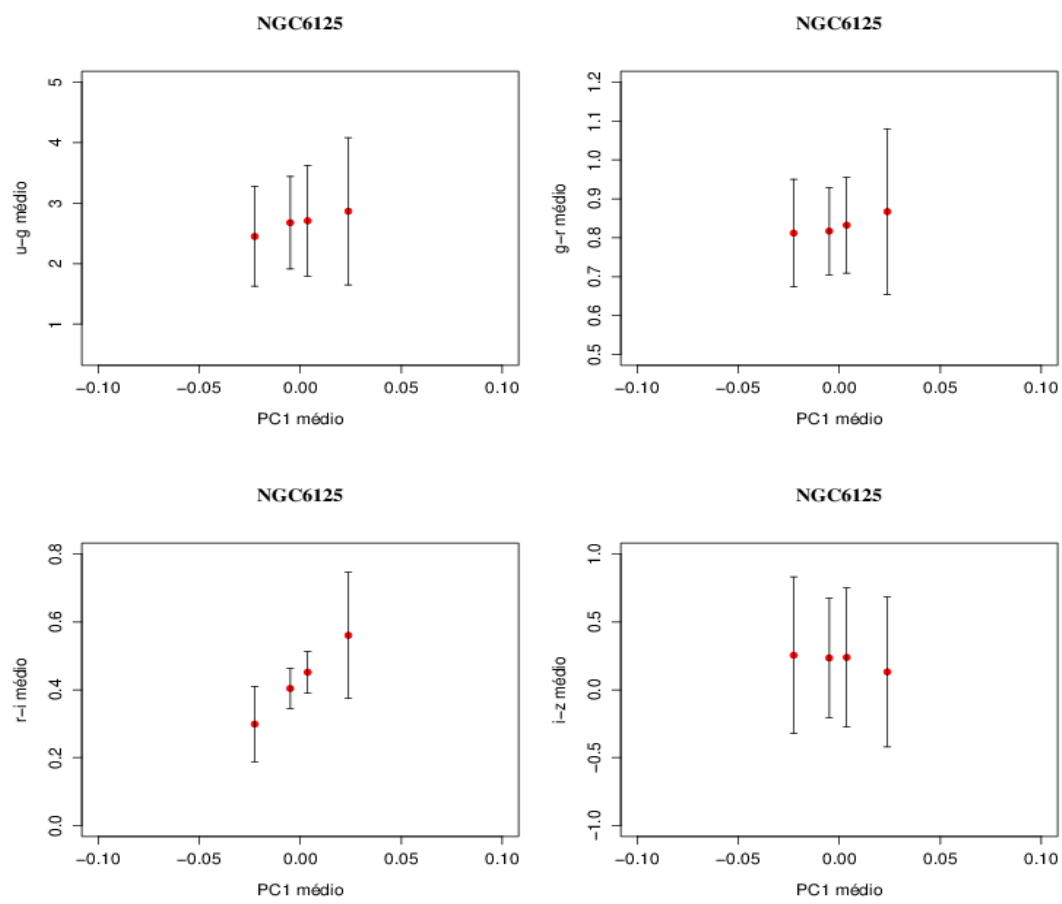

Figura A.2: Análise PCA nos píxeis: As cores $u-g, g-r, r-i$ e $i$ - $z$ em função da $1^{a}$ componente principal, para NGC6125. 

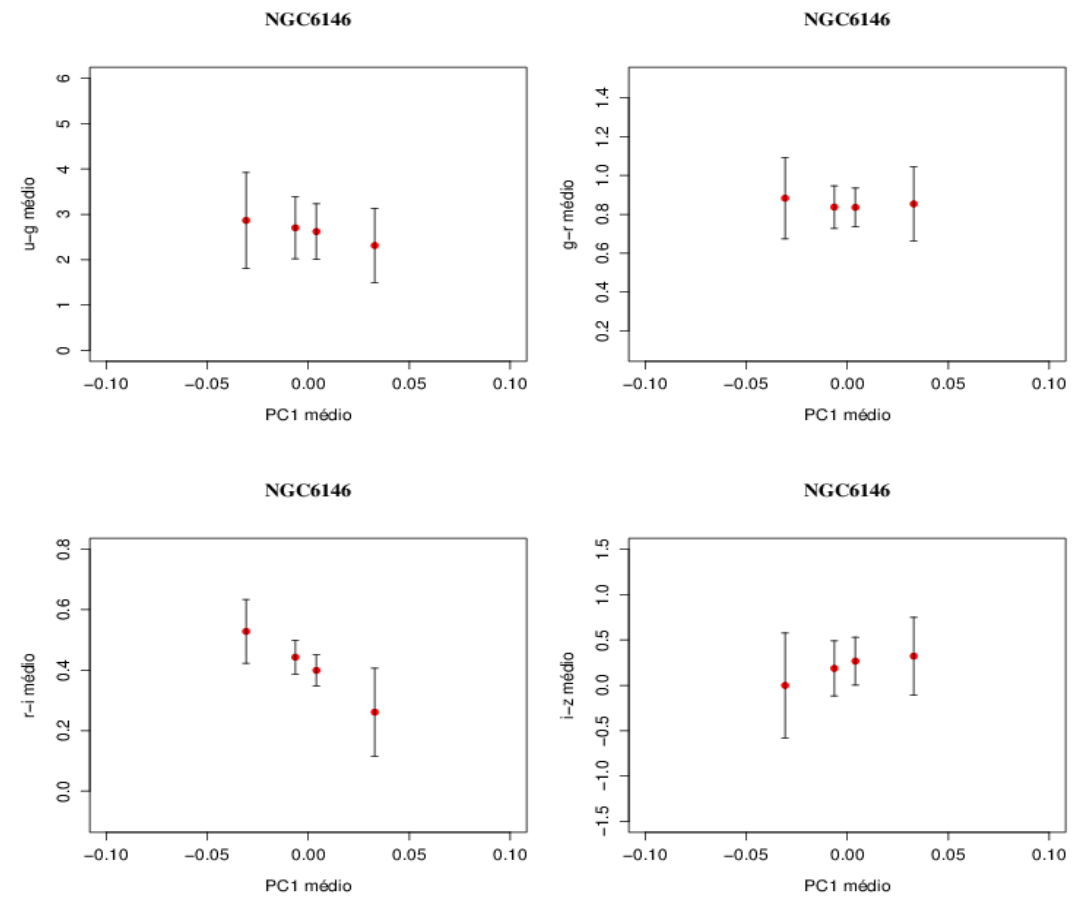

Figura A.3: Análise PCA nos píxeis: As cores $u-g, g-r, r-i$ e $i$-z em função da $1^{a}$ componente principal, para NGC6146.
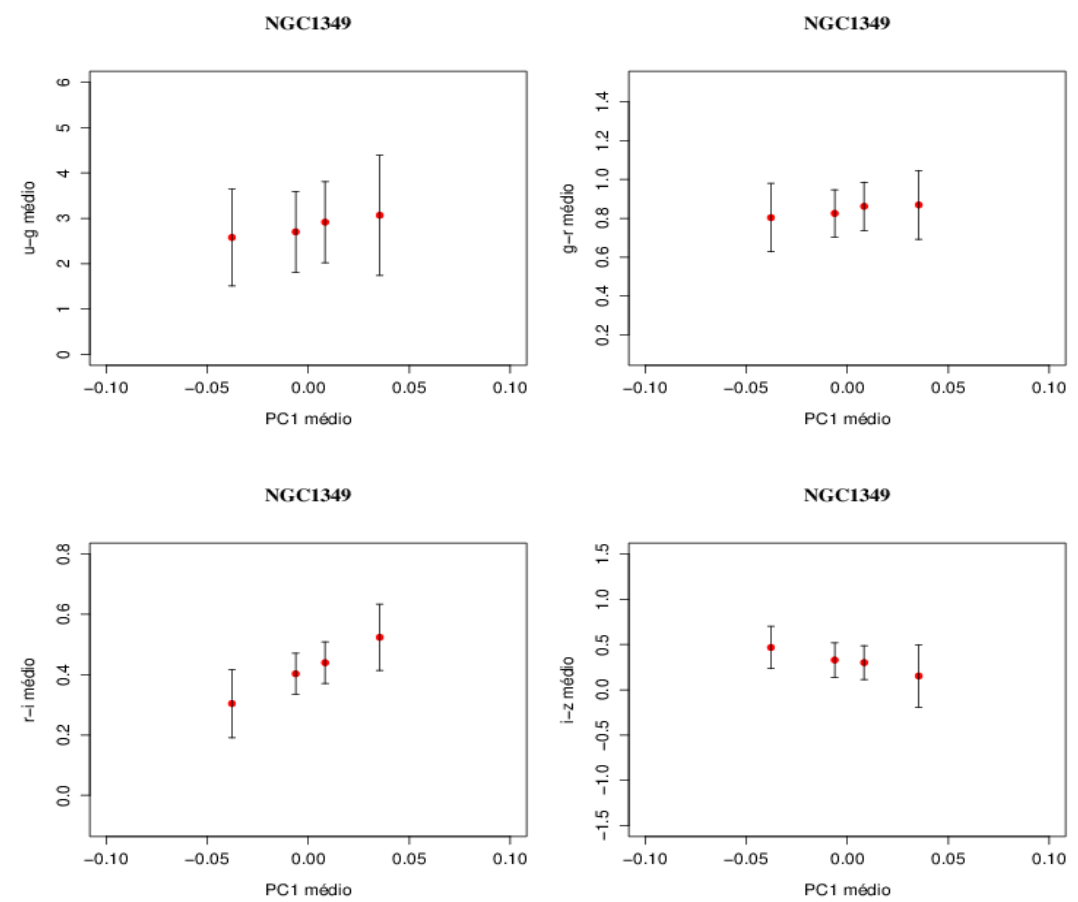

Figura A.4: Análise PCA nos píxeis: As cores $u-g, g-r, r-i$ e $i$-z em função da $1^{a}$ componente principal, para NGC1349. 

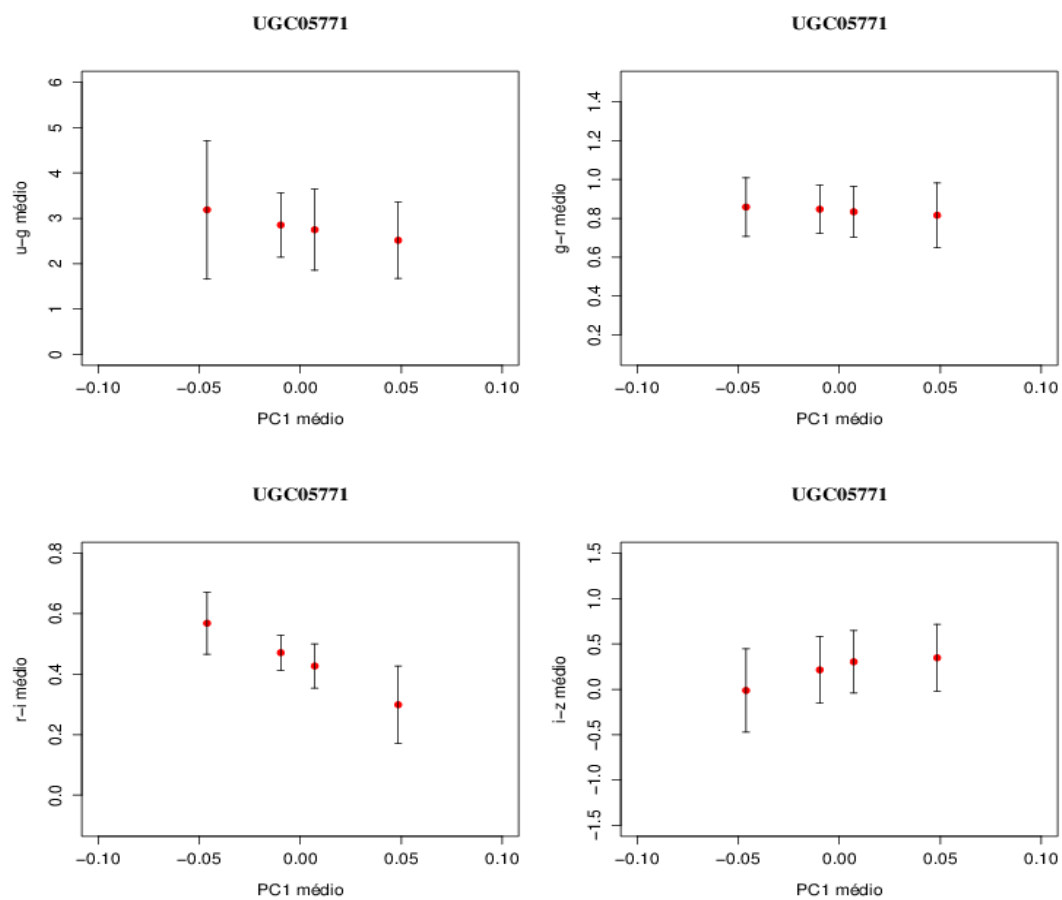

Figura A.5: Análise PCA nos píxeis: As cores $u-g, g-r, r-i$ e $i-z$ em função da $1^{a}$ componente principal, para UGC05771.

\section{A.1.2 Galáxias Espirais}

PCA em função das cores $u-g, g-r, r-i$ e $i-z$ para as 4 populações estelares, nas galáxias da subamostra de galáxias espirais. 

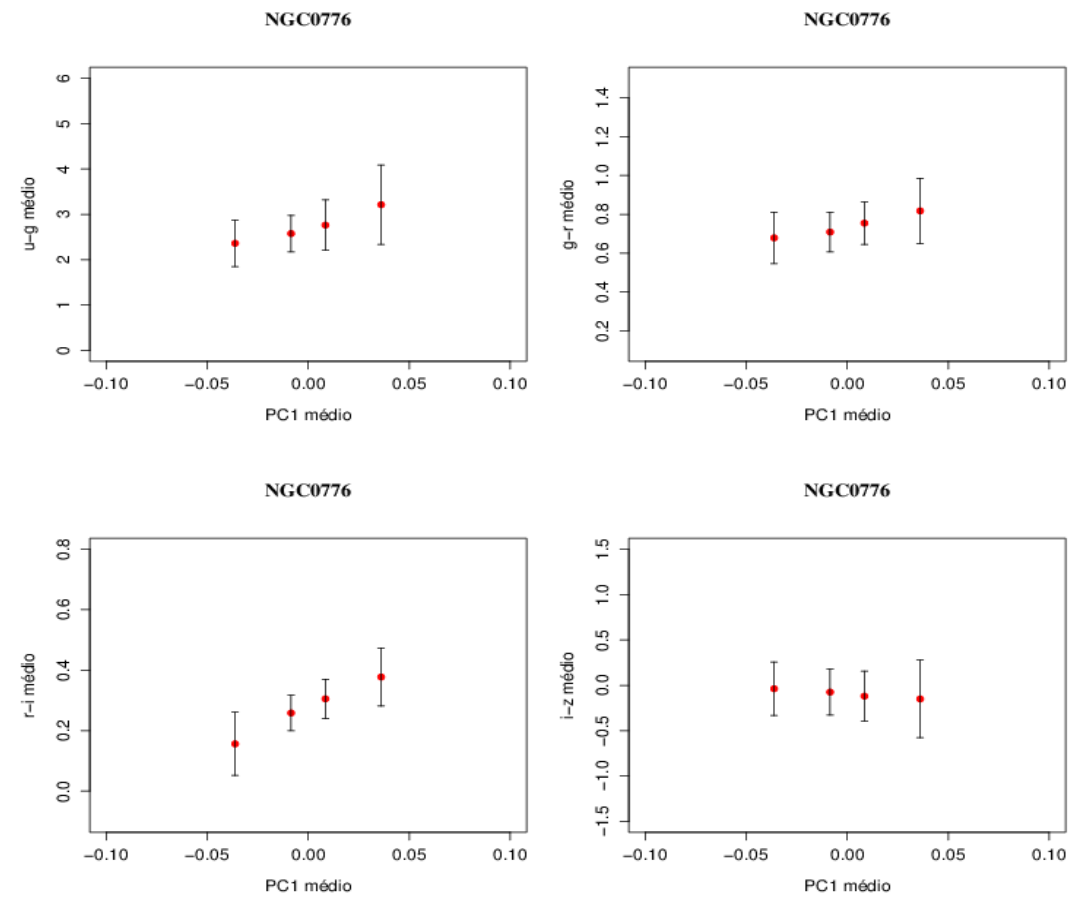

Figura A.6: Análise PCA nos píxeis: As cores $u-g, g-r, r-i$ e $i$-z em função da $1^{a}$ componente principal, para NGC0776.
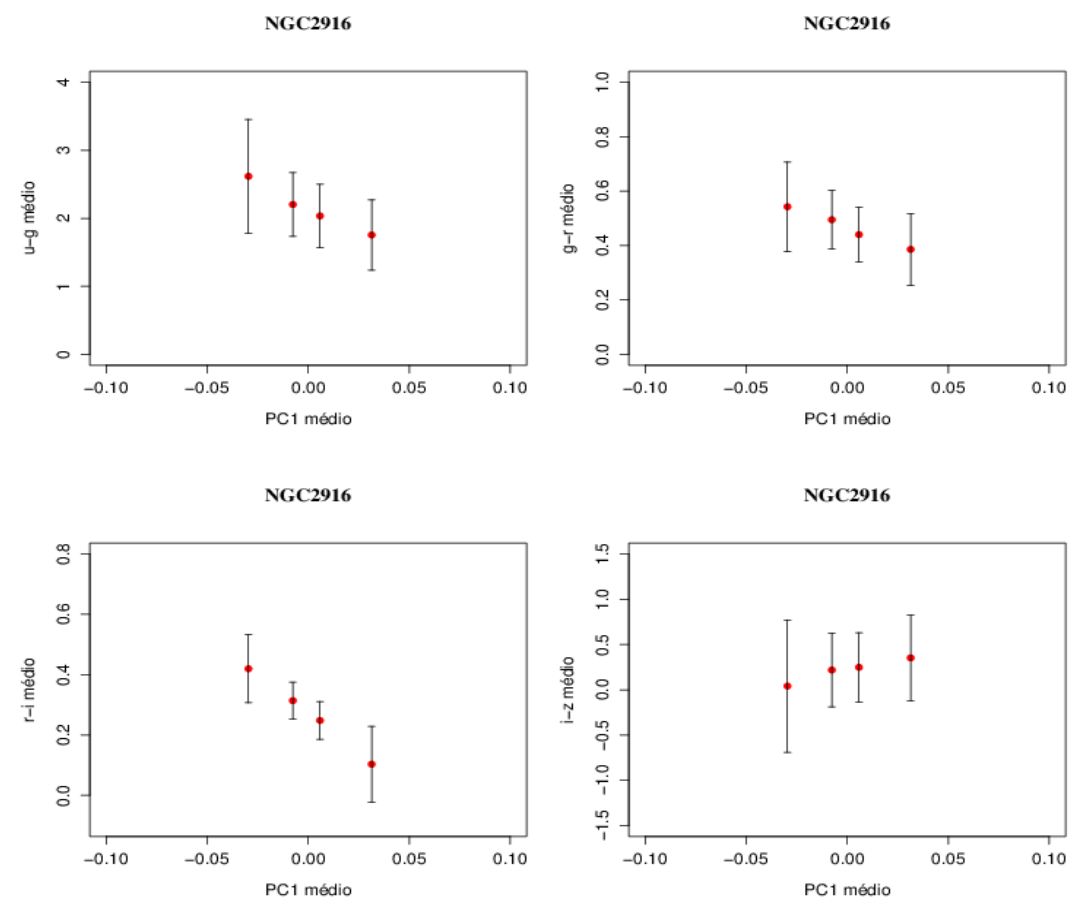

Figura A.7: Análise PCA nos píxeis: As cores $u-g, g-r, r-i$ e $i$-z em função da $1^{a}$ componente principal, para NGC2916. 

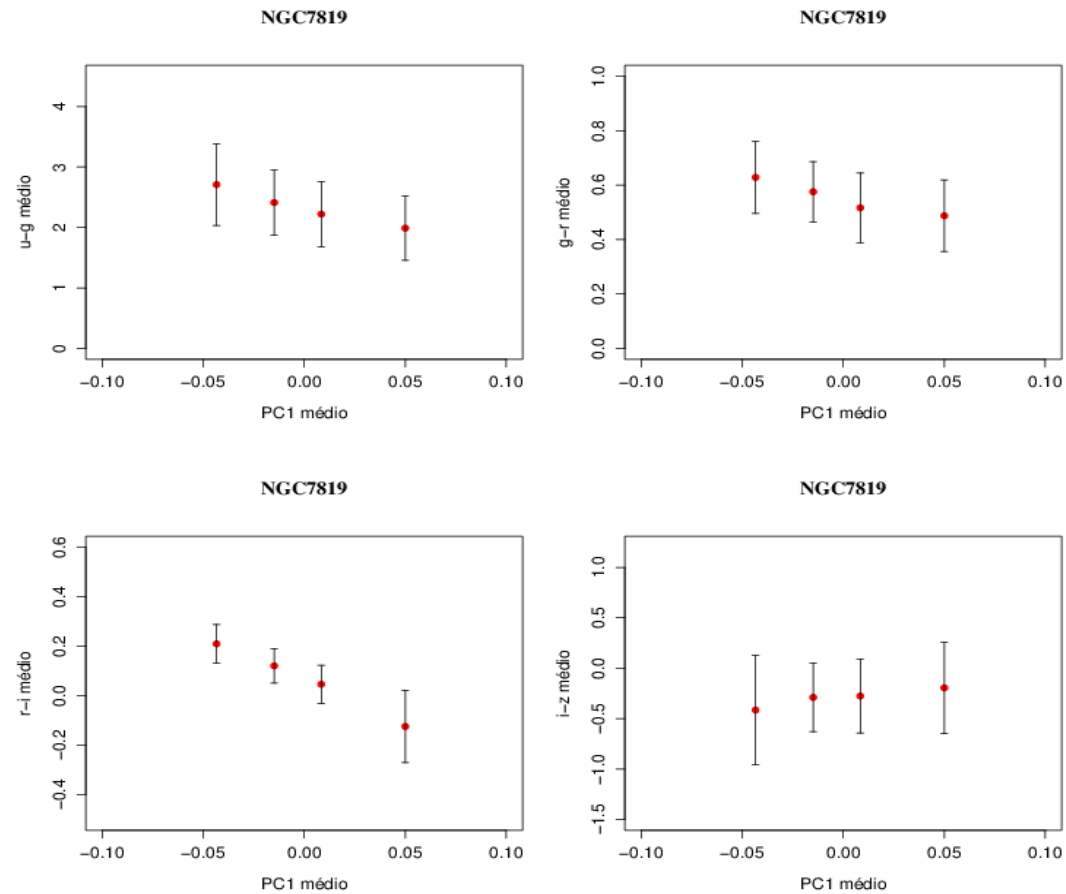

Figura A.8: Análise PCA nos píxeis: As cores $u-g, g-r, r-i$ e $i-z$ em função da $1^{a}$ componente principal, para NGC7819.
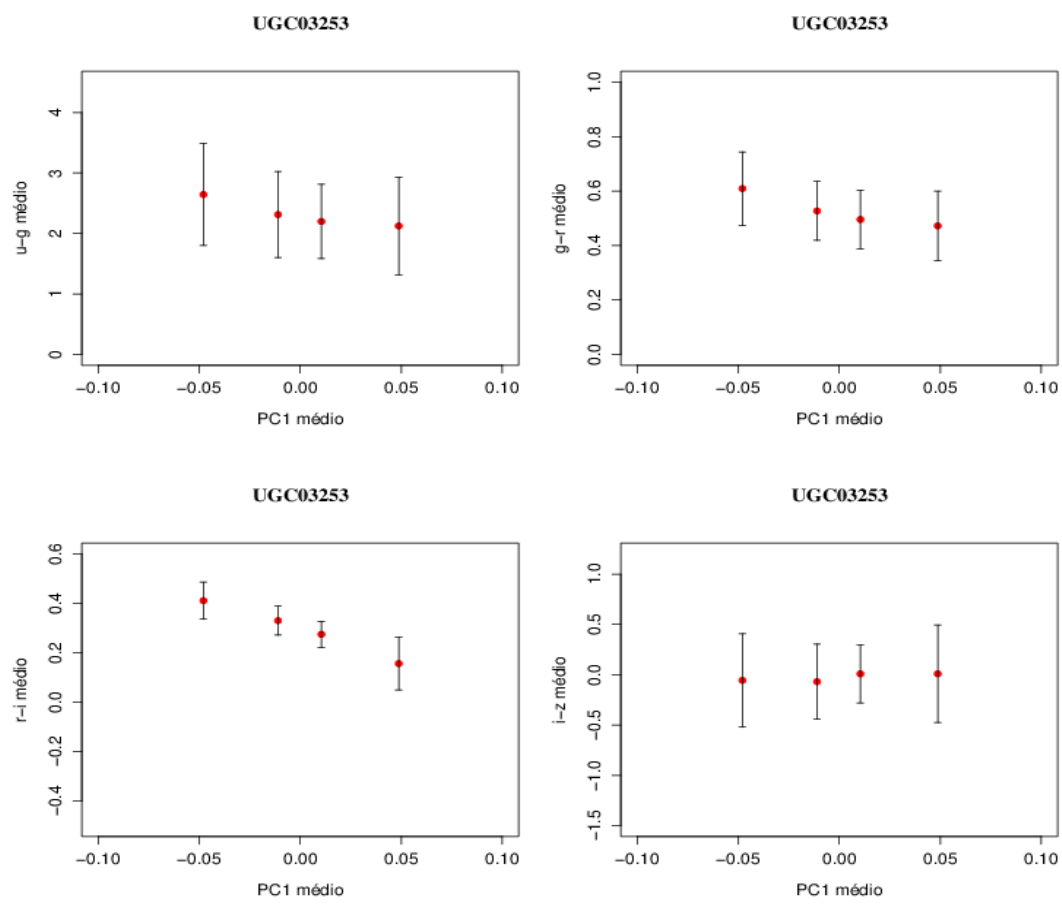

Figura A.9: Análise PCA nos píxeis: As cores $u-g, g-r, r-i$ e $i$ - $z$ em função da $1^{a}$ componente principal, para UGC03253. 

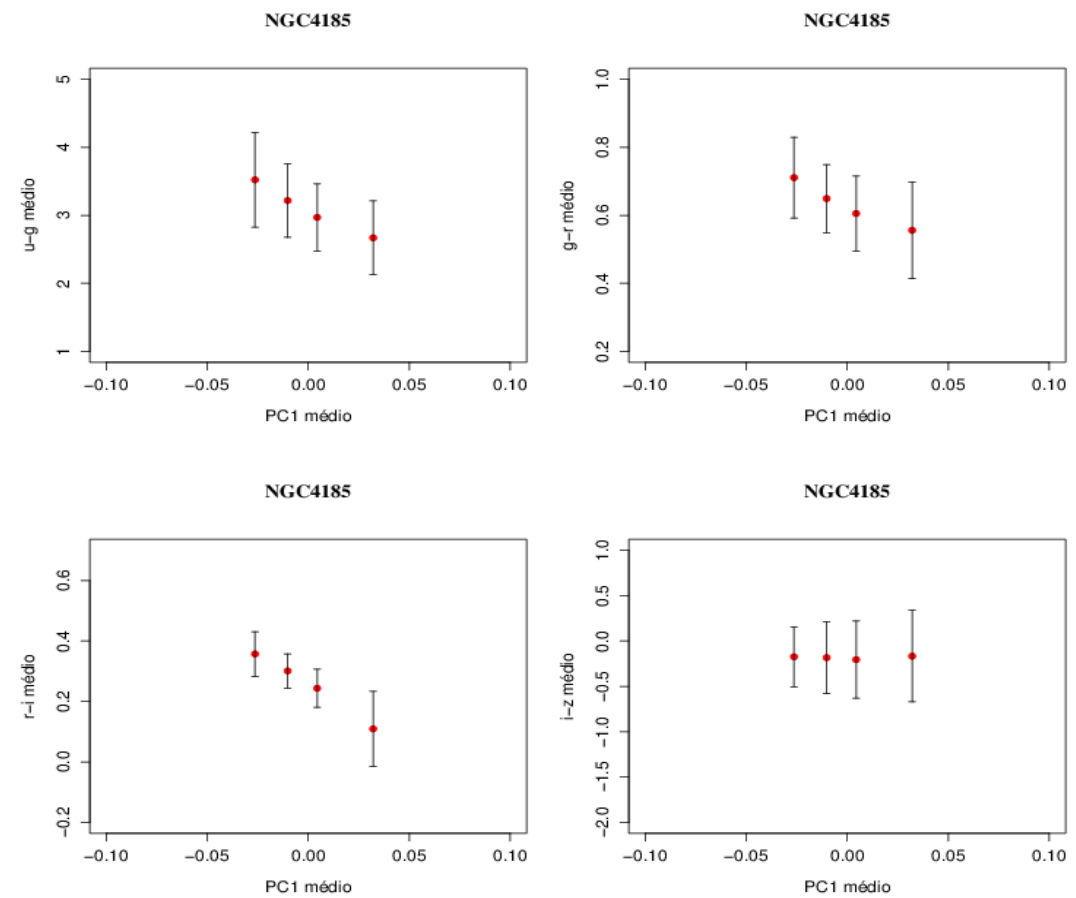

Figura A.10: Análise PCA nos píxeis: As cores $u-g, g-r, r-i$ e $i$ - $z$ em função da $1^{a}$ componente principal, para NGC4185.

\section{A.1.3 Galáxias Espirais Tardias}

PCA em função das cores $u-g, g-r, r-i$ e $i-z$ para as 4 populações estelares, nas galáxias da subamostra de galáxias espirais tardias. 

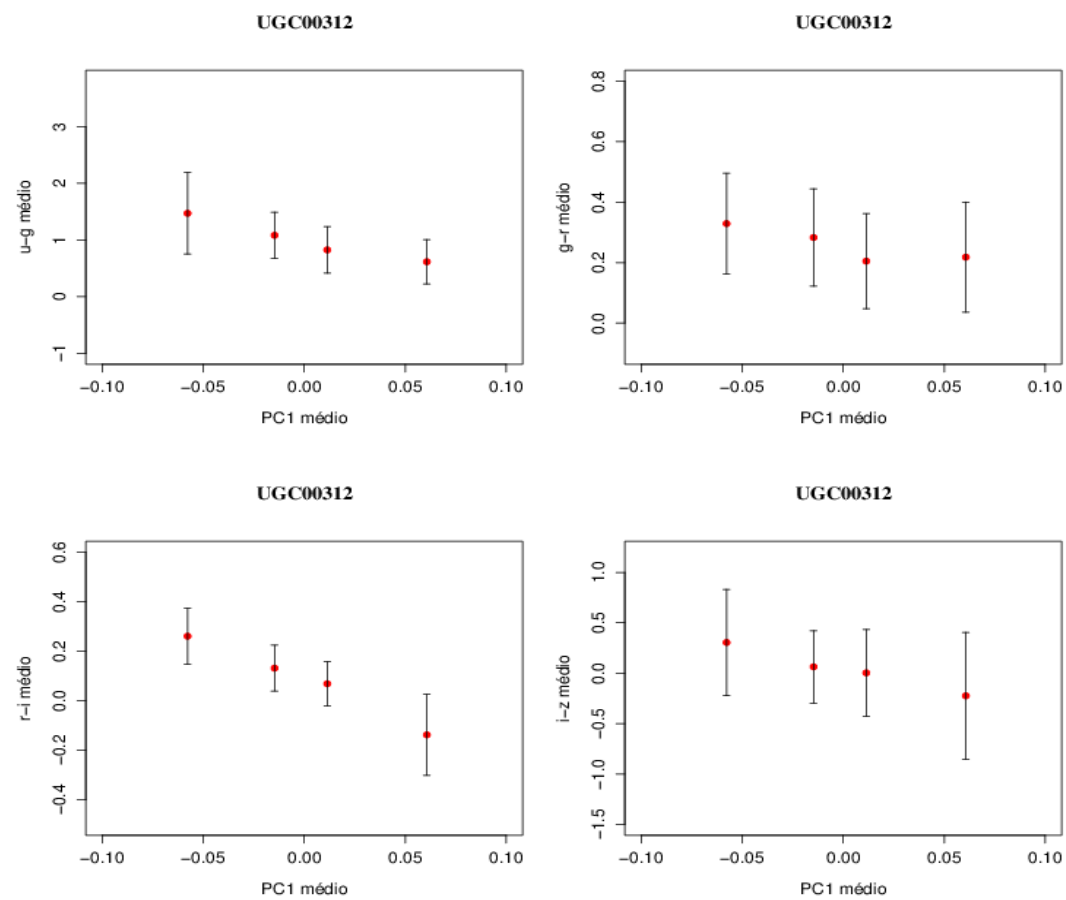

Figura A.11: Análise PCA nos píxeis: As cores $u-g, g-r, r-i$ e $i$ - $z$ em função da $1^{a}$ componente principal, para UGC00312.
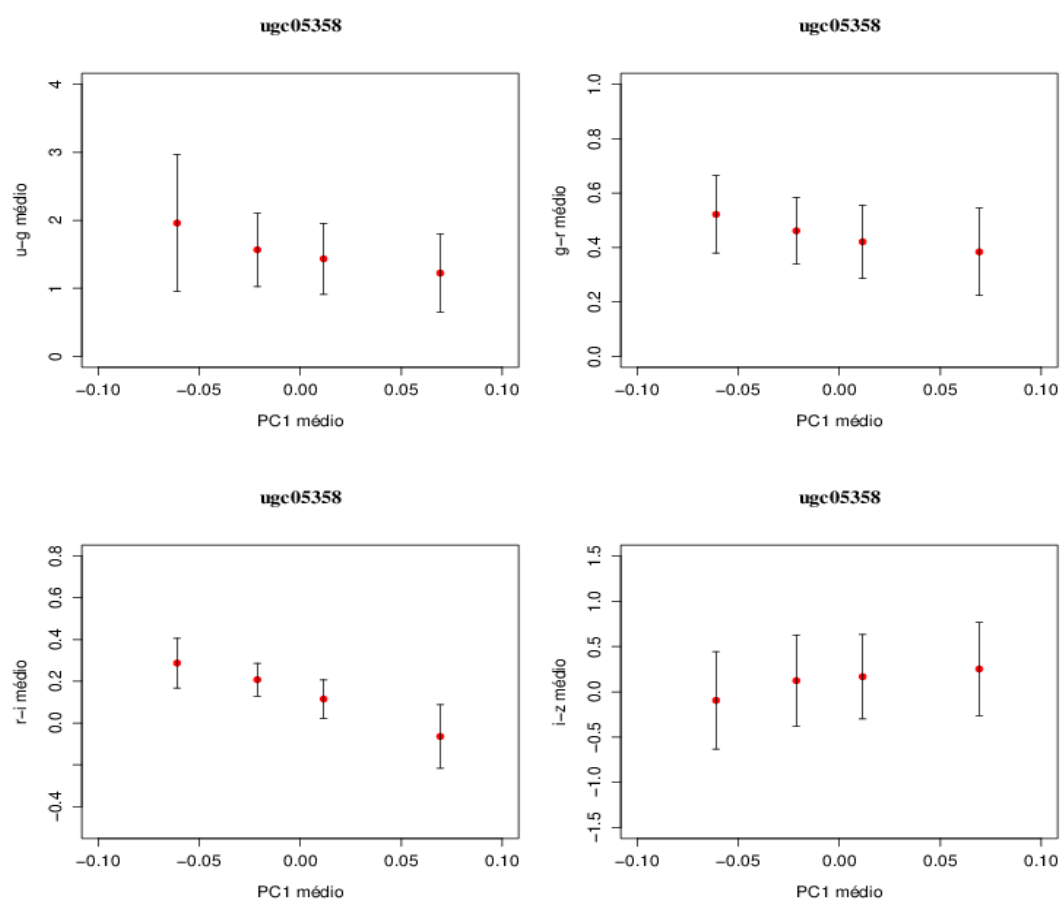

Figura A.12: Análise PCA nos píxeis: As cores $u-g, g-r, r-i$ e $i-z$ em função da $1^{a}$ componente principal, para UGC05358. 

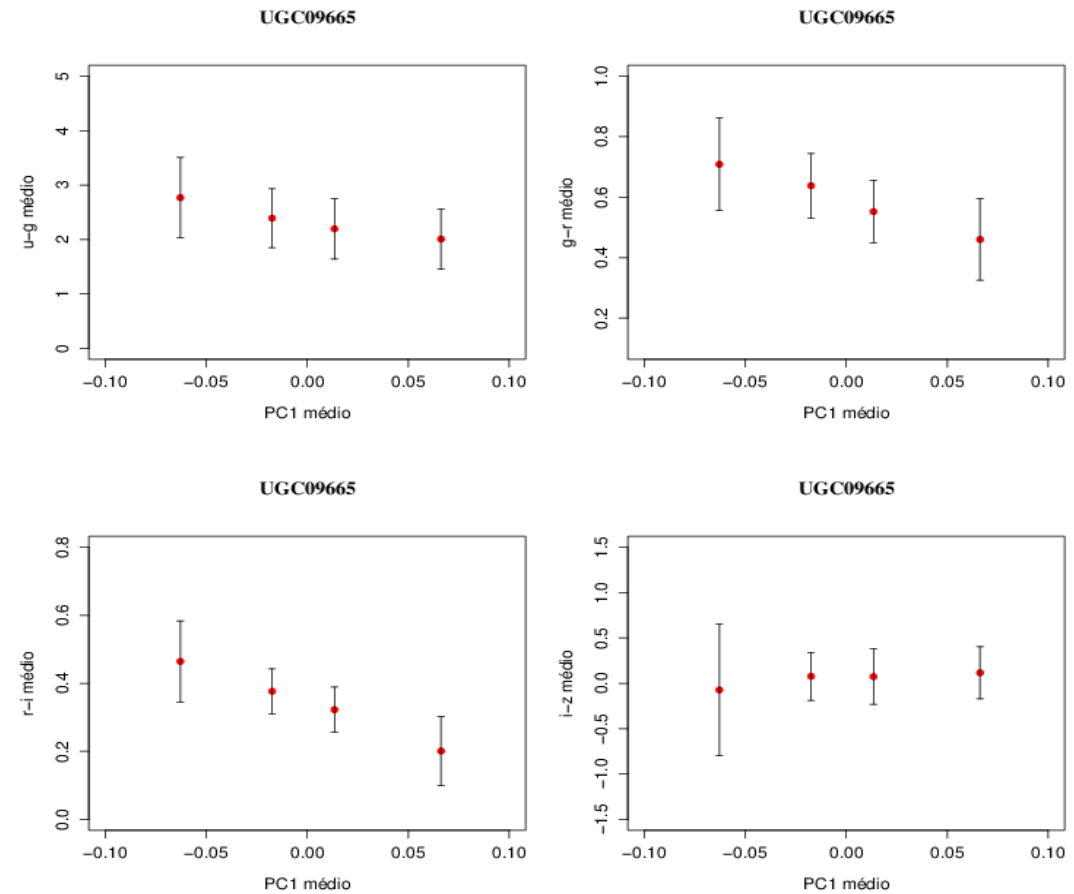

Figura A.13: Análise PCA nos píxeis: As cores $u-g, g-r, r-i$ e $i-z$ em função da $1^{a}$ componente principal, para UGC09665.
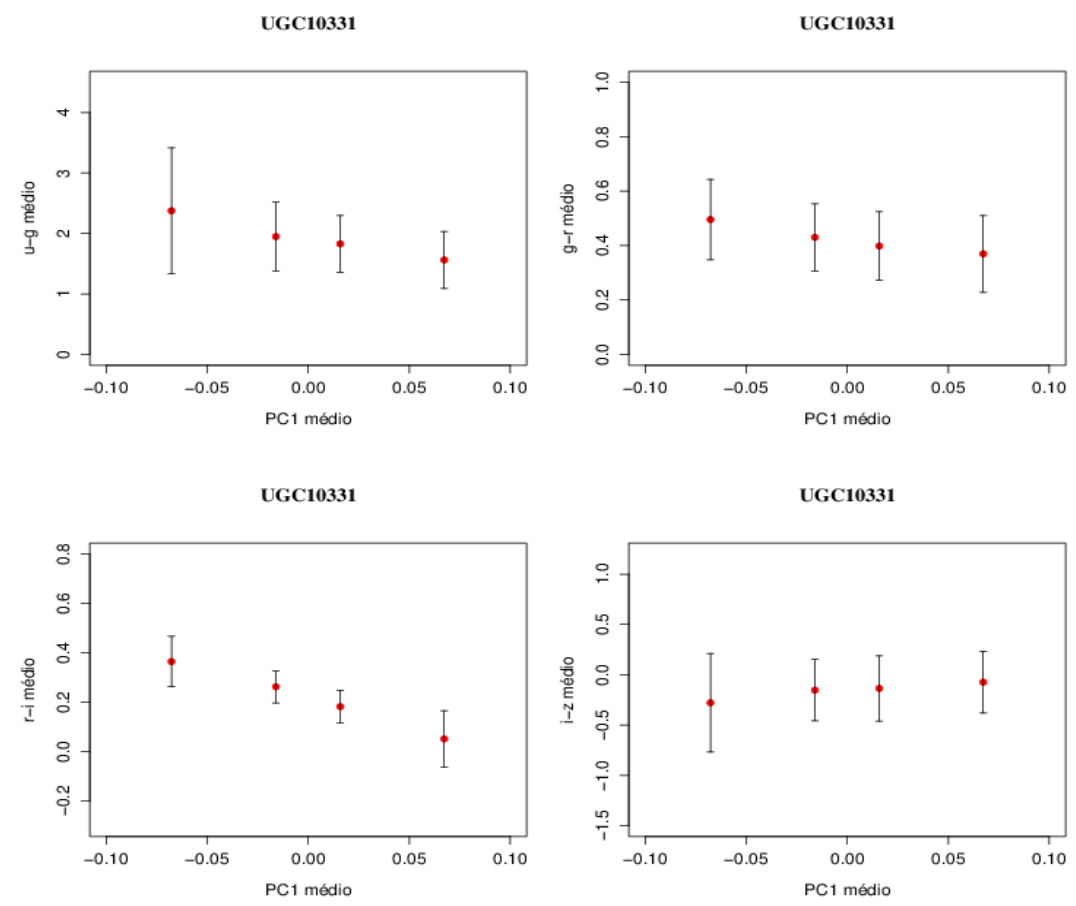

Figura A.14: Análise PCA nos píxeis: As cores $u-g, g-r, r-i$ e $i-z$ em função da $1^{a}$ componente principal, para UGC10331. 

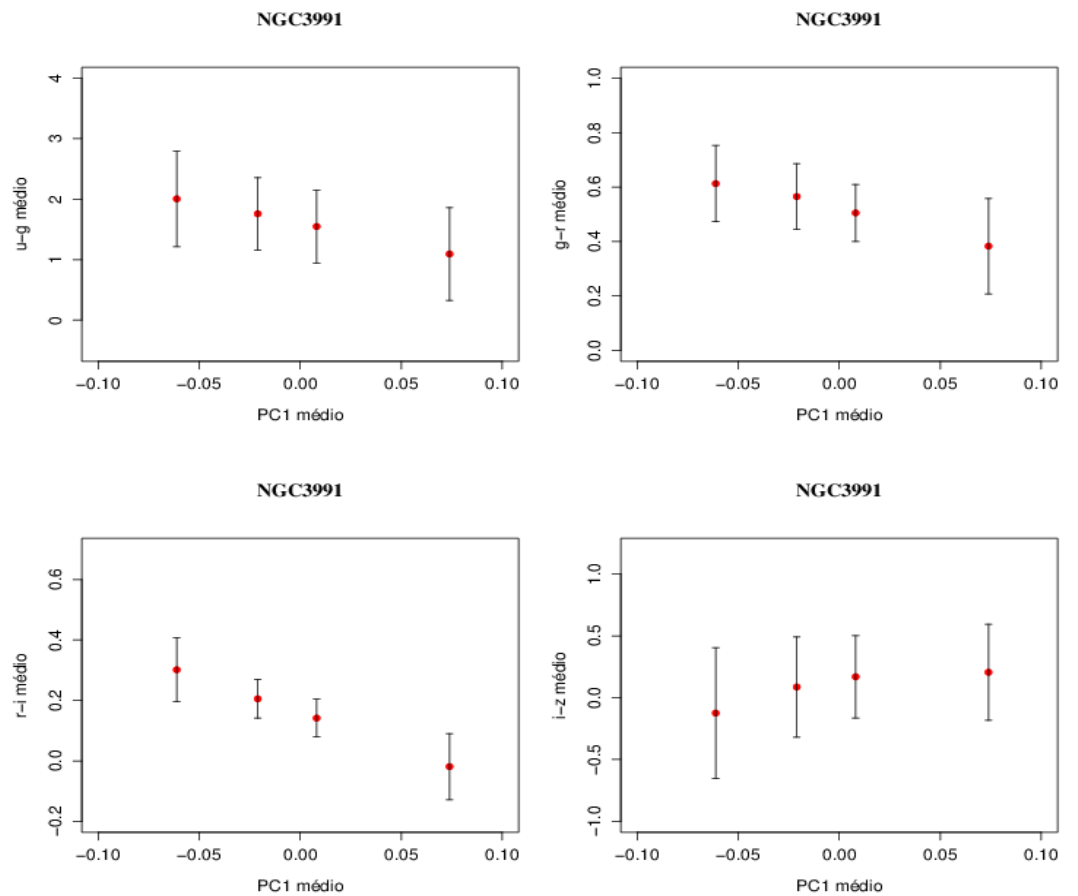

Figura A.15: Análise PCA nos píxeis: As cores $u-g, g-r, r-i$ e $i$ - $z$ em função da $1^{a}$ componente principal, para NGC3991.

\section{A.2 Distribuição Espacial}

Abaixo estão as distribuições espacias de cada população de cada galáxia. 


\section{A.2.1 Galáxias Elípticas}
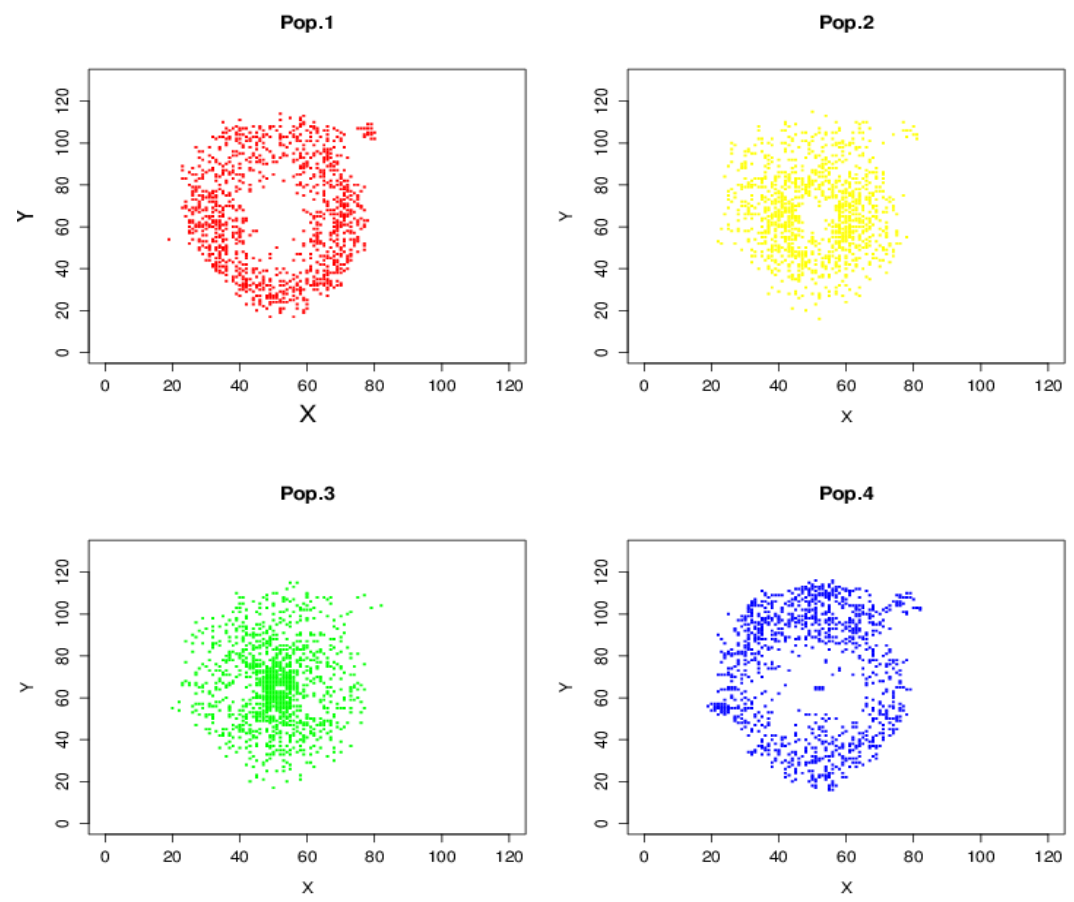

Figura A.16: NGC6173
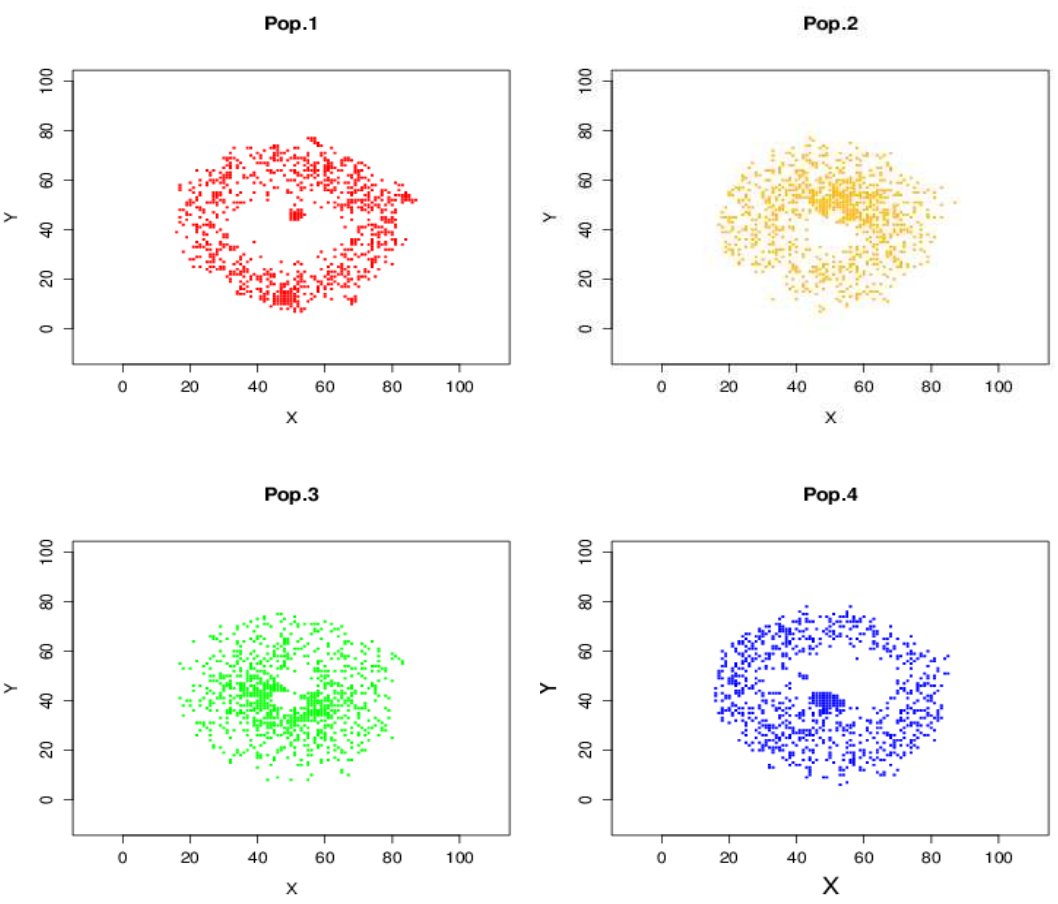

Figura A.17: NGC6125 

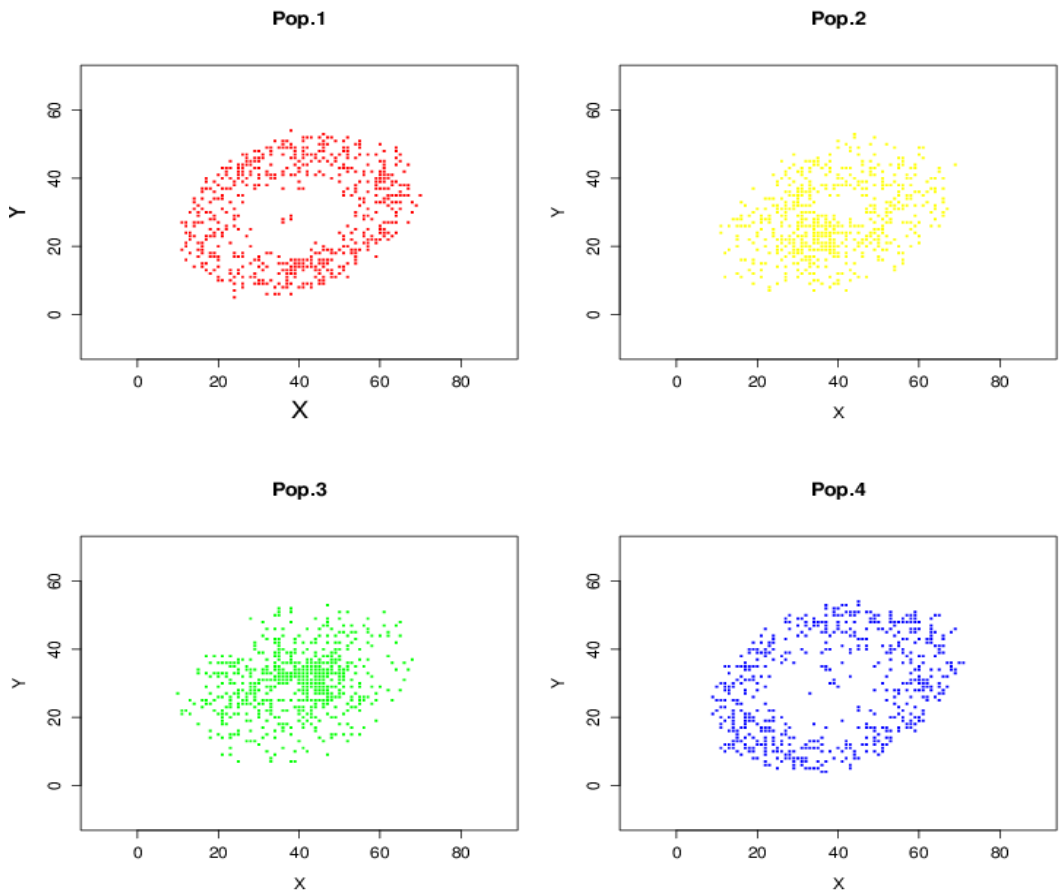

Figura A.18: NGC6146
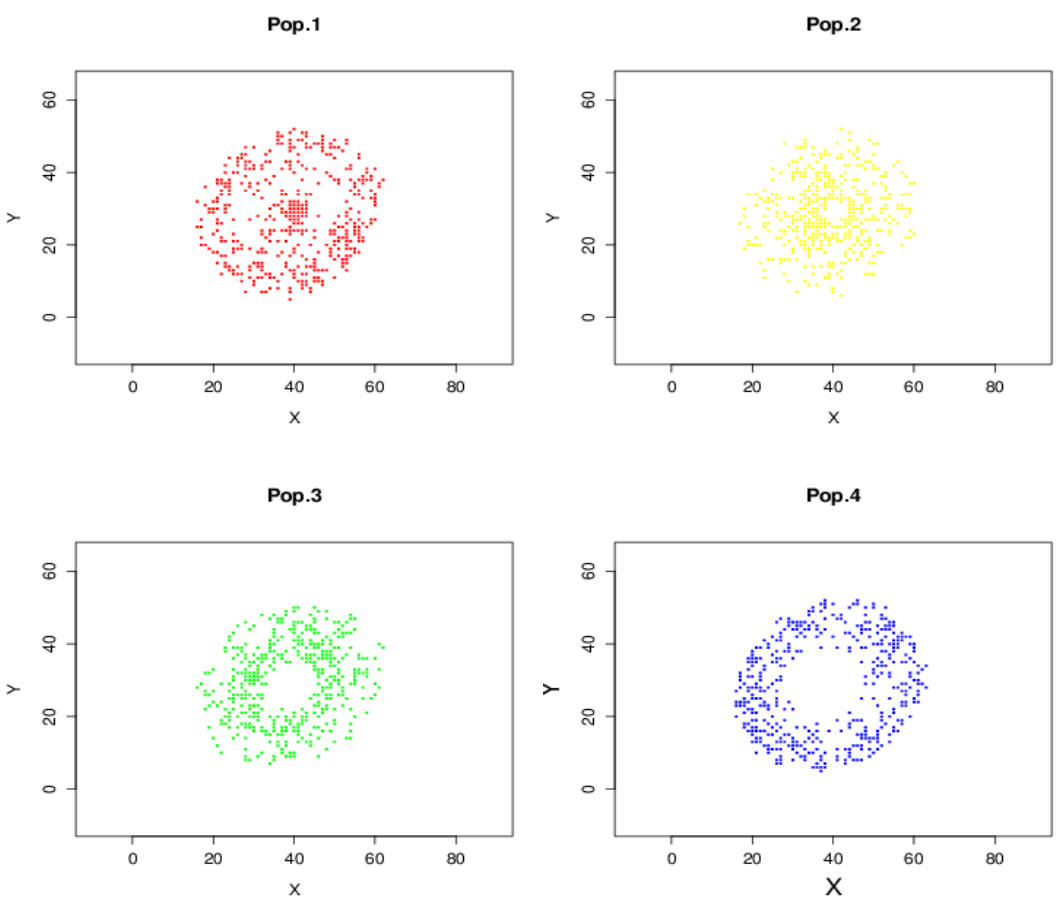

Figura A.19: NGC1349 

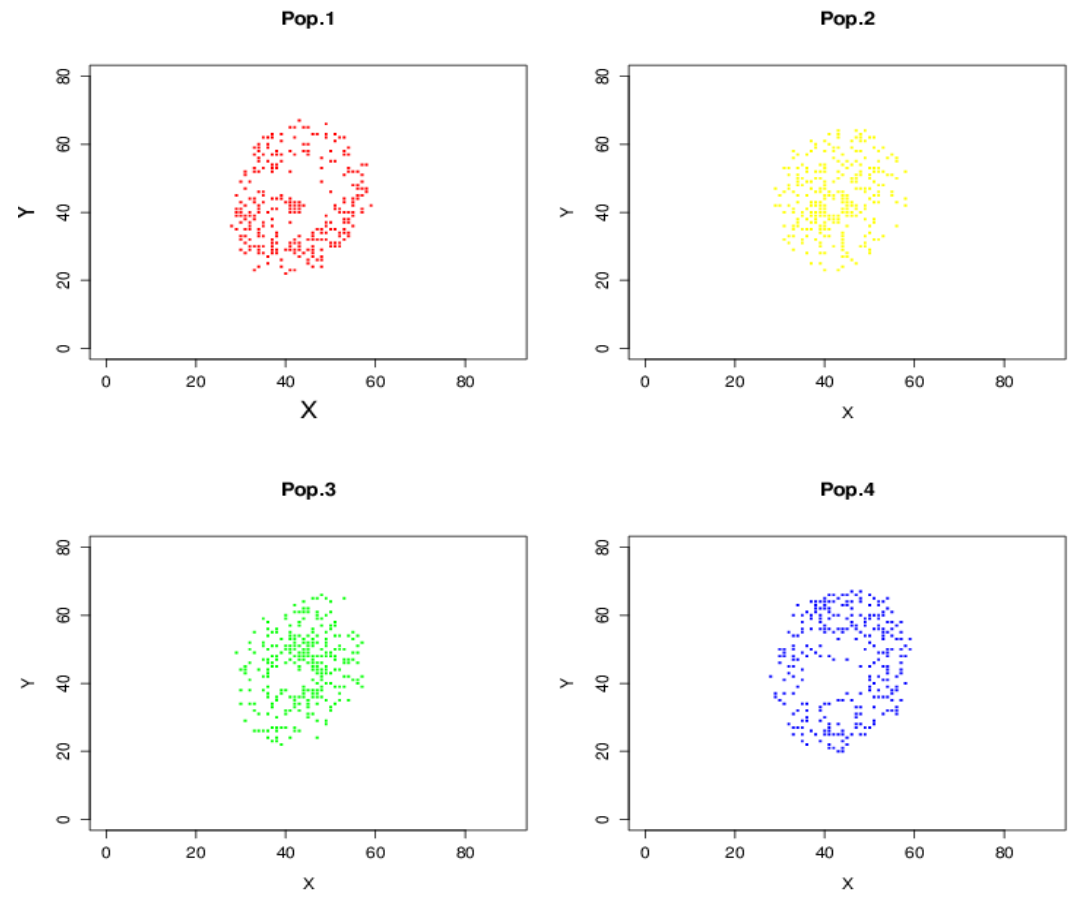

Figura A.20: UGC05771

\section{A.2.2 Galáxias Espirais}

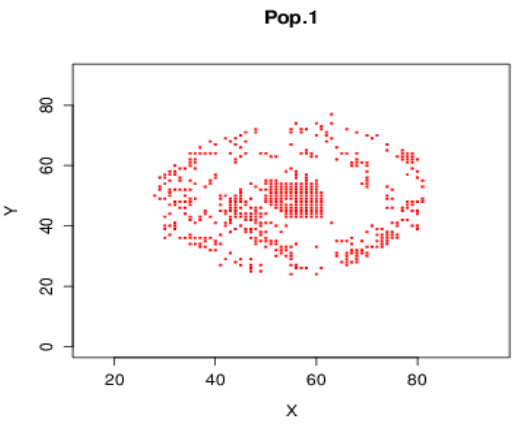

Pop.3

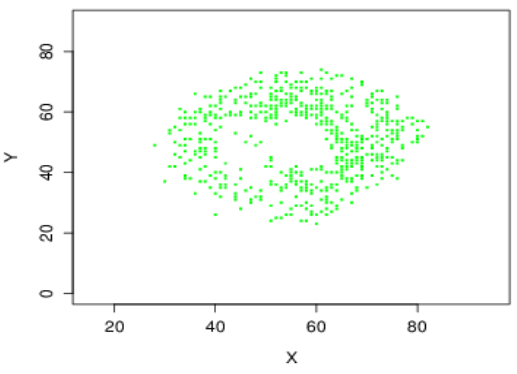

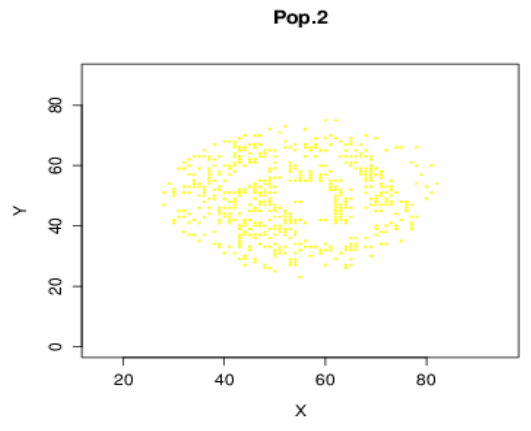

Pop.4

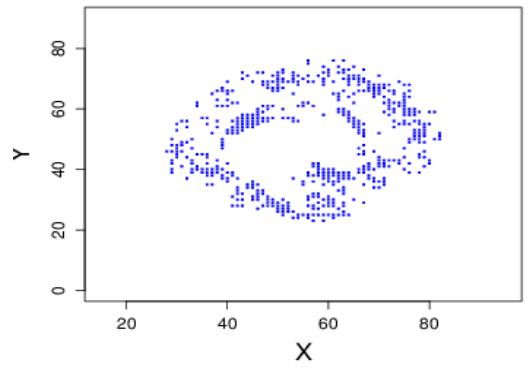

Figura A.21: NGC0776 

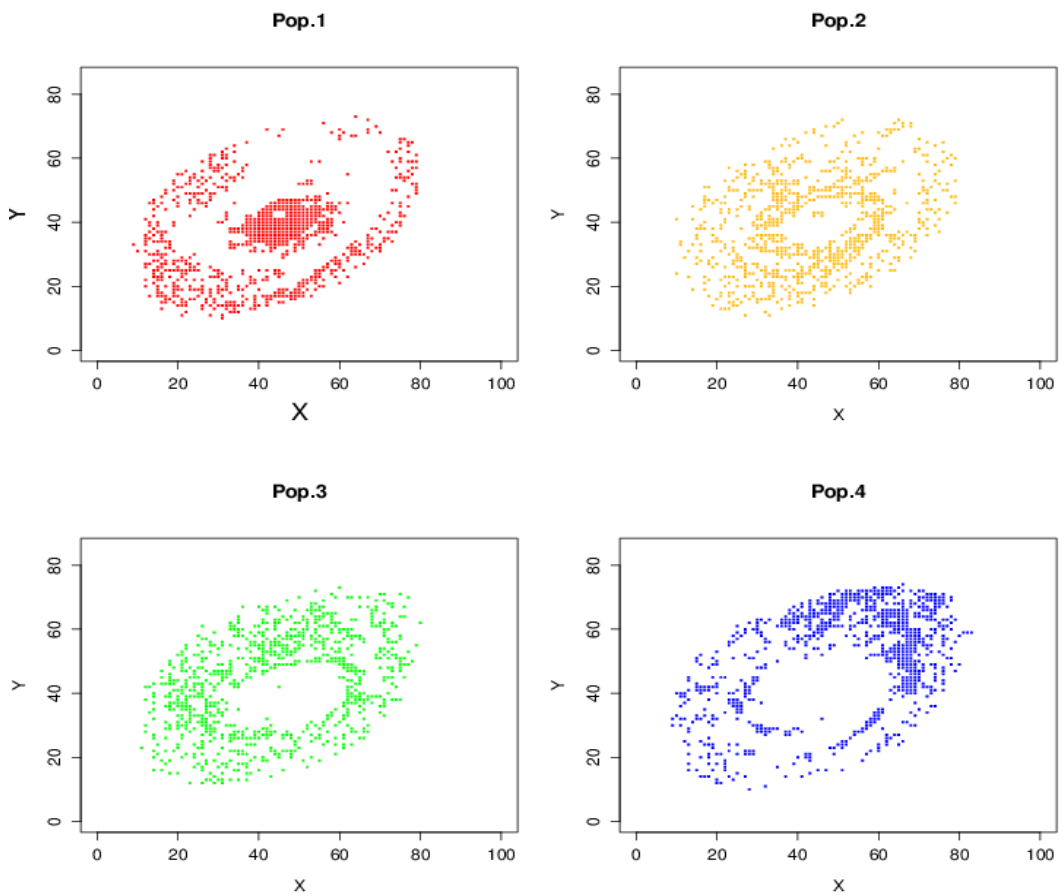

Figura A.22: NGC2916
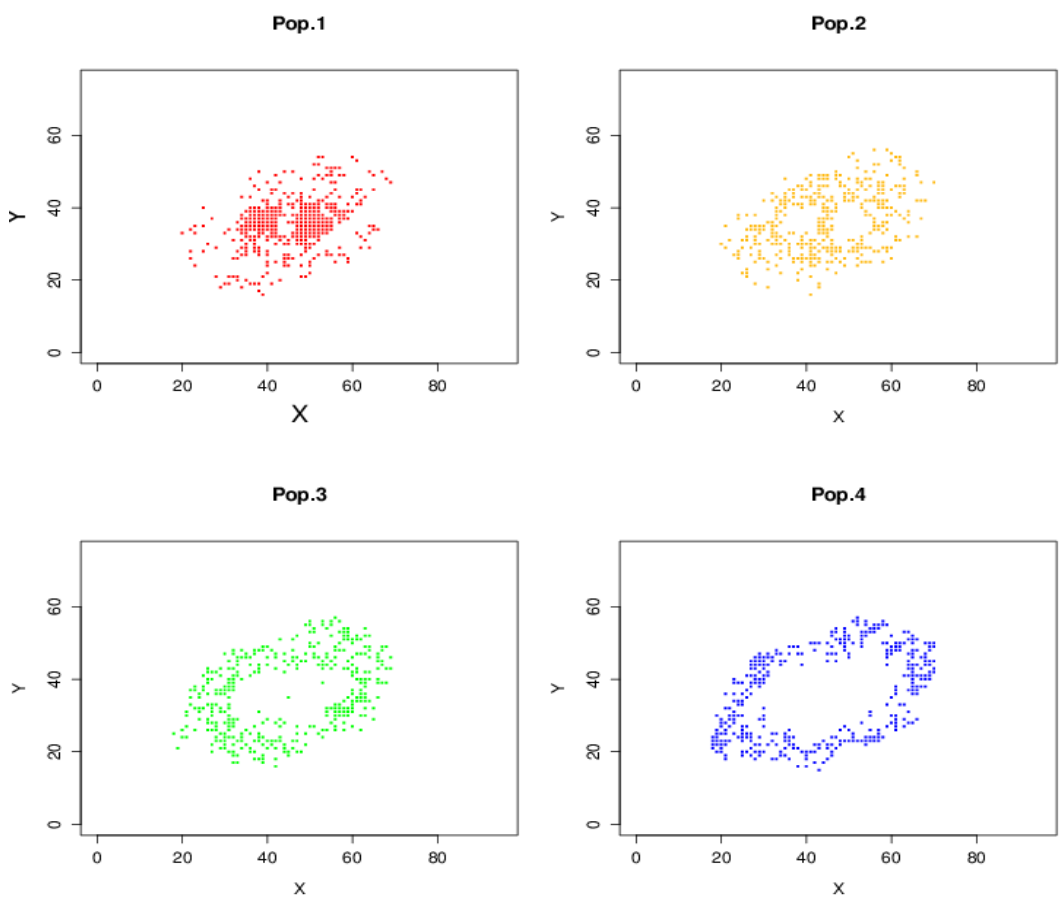

Figura A.23: NGC7819 

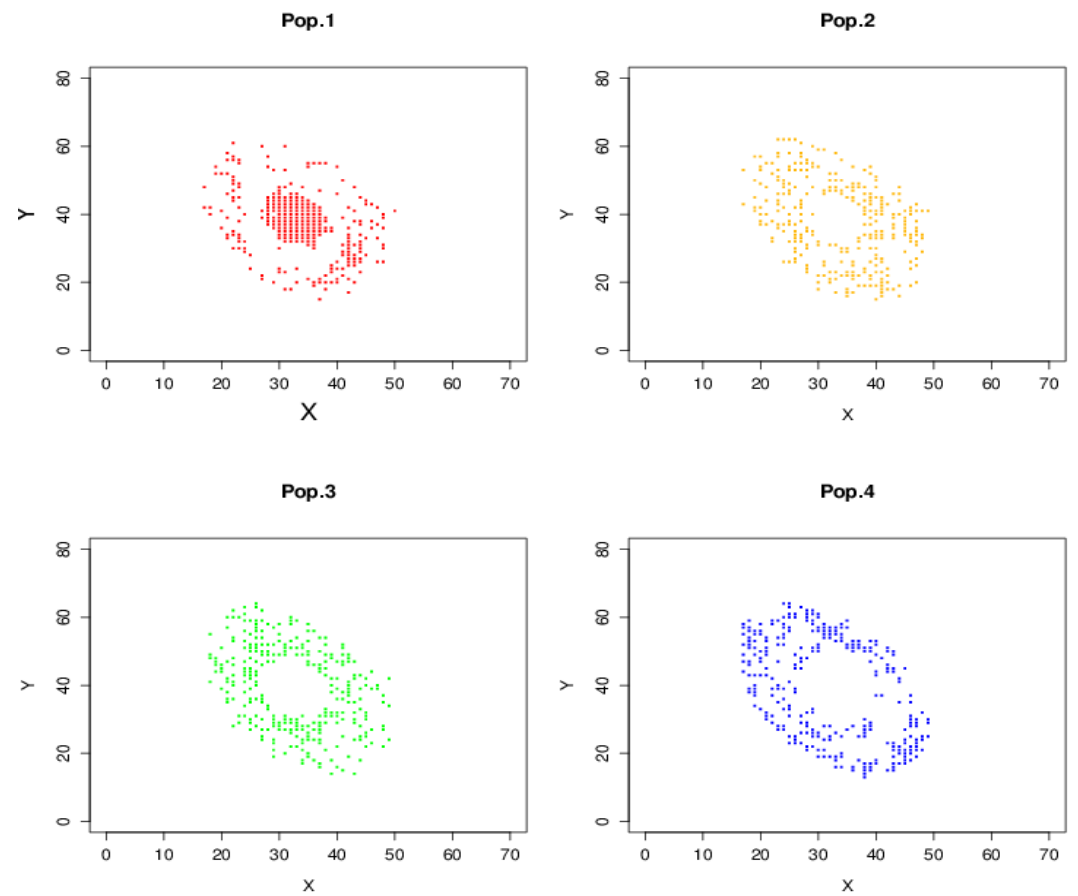

Figura A.24: UGC03253
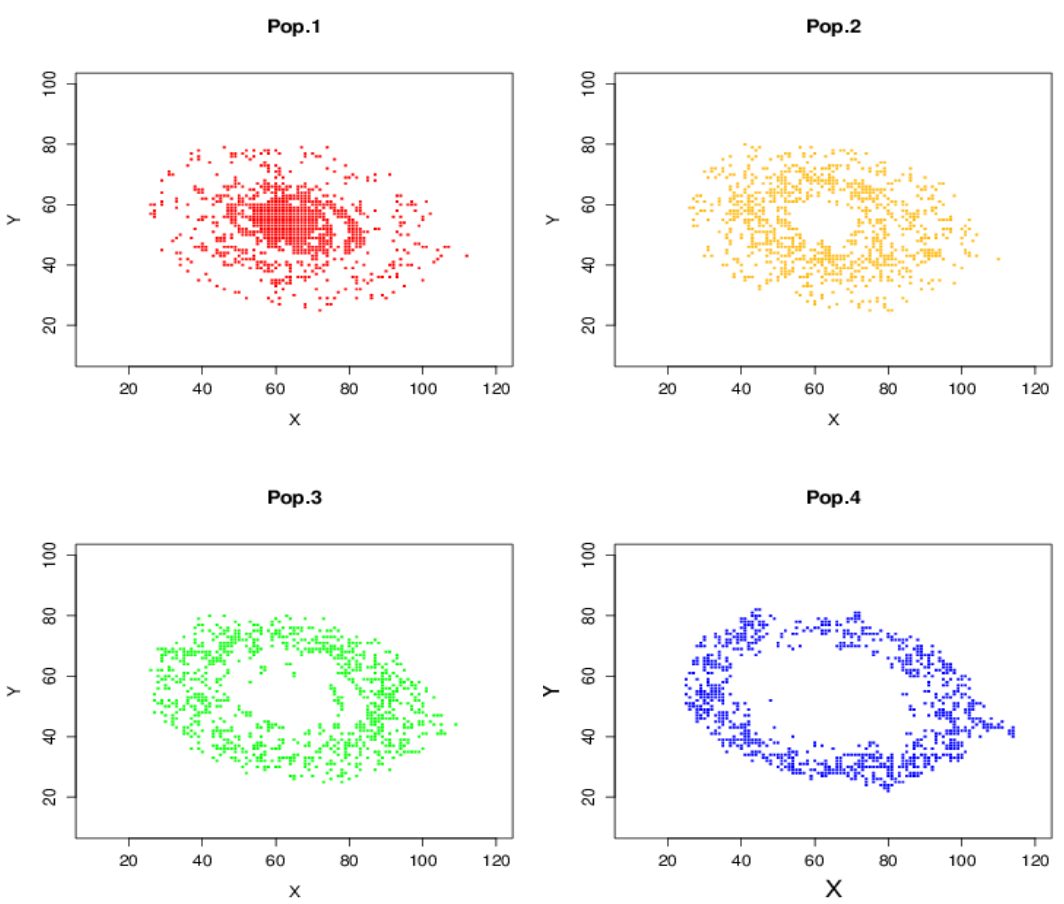

Figura A.25: NGC4185 


\section{A.2.3 Galáxias Espirais Tardias}
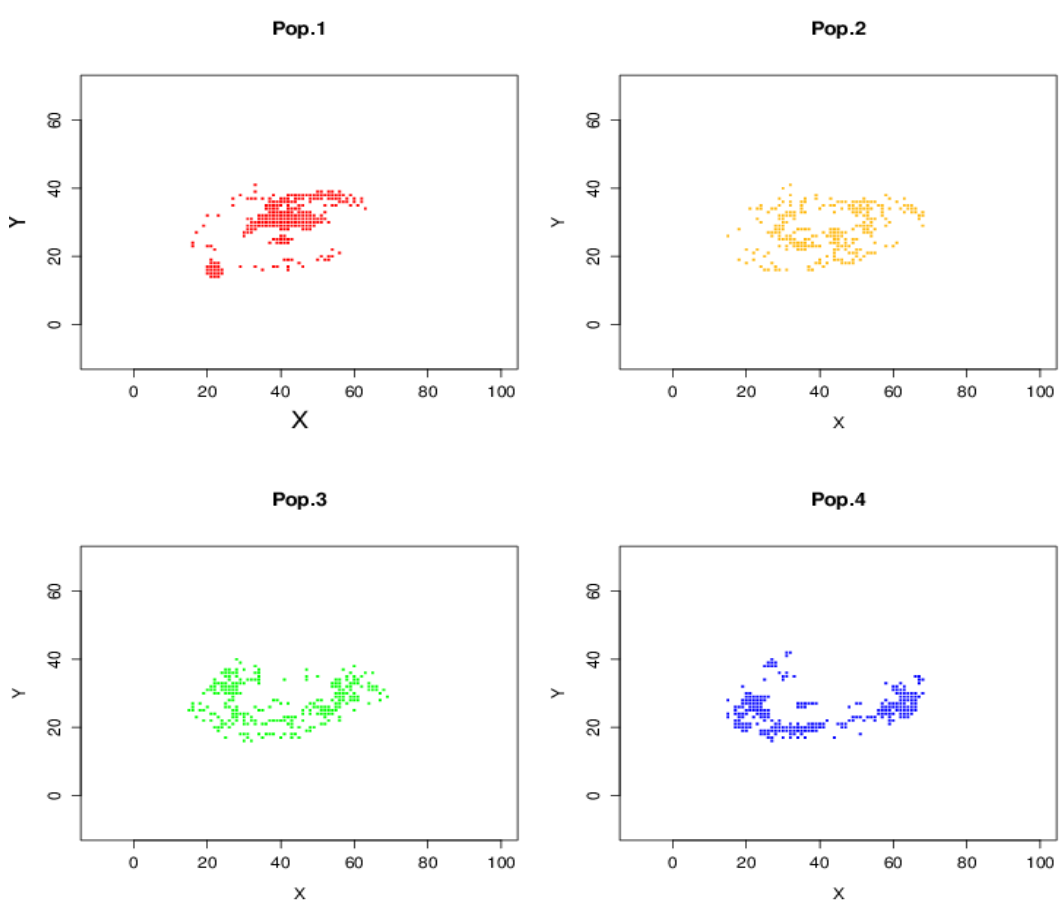

Figura A.26: UGC00312
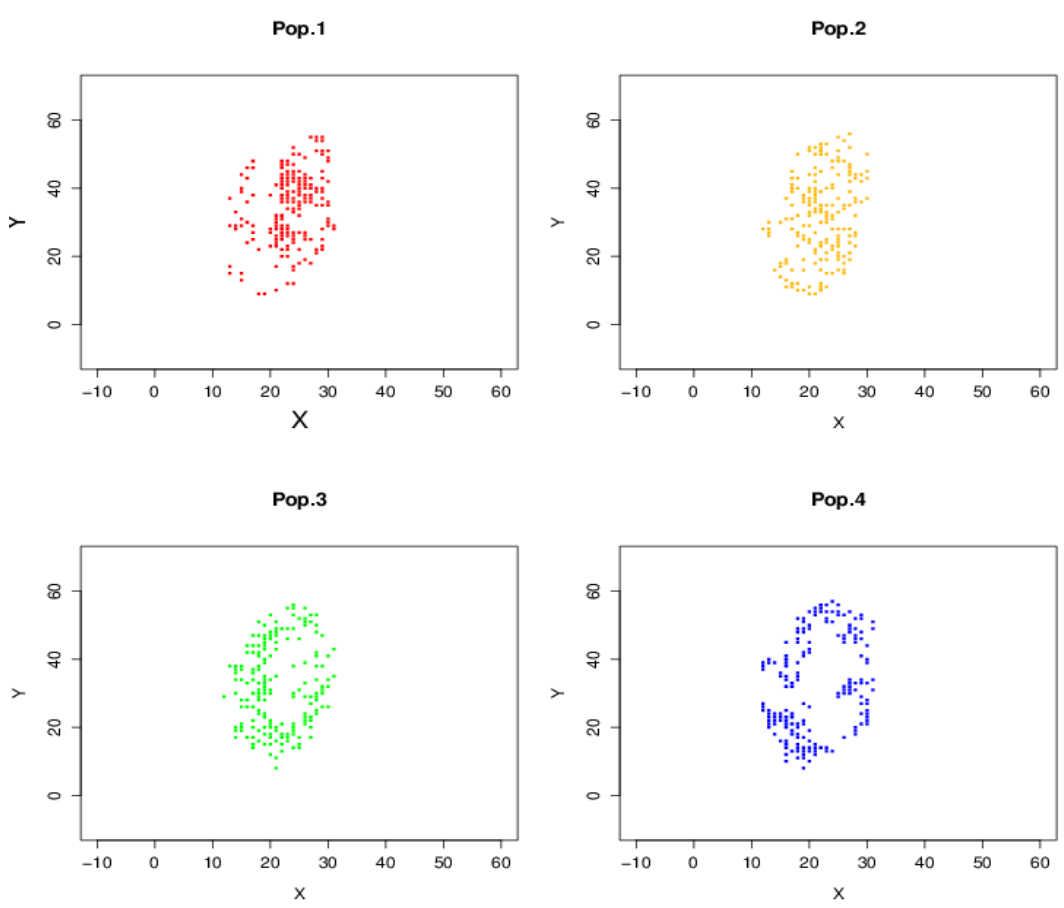

Figura A.27: UGC05358 

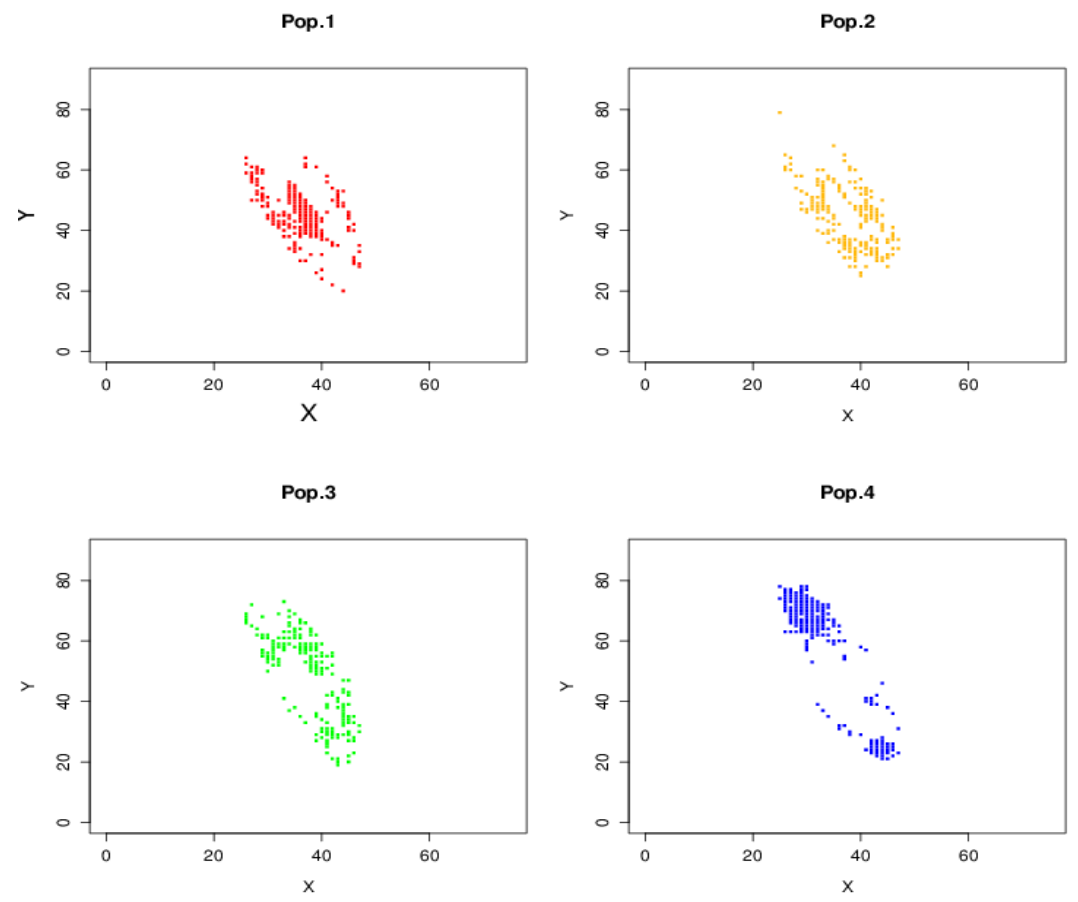

Figura A.28: UGC09665
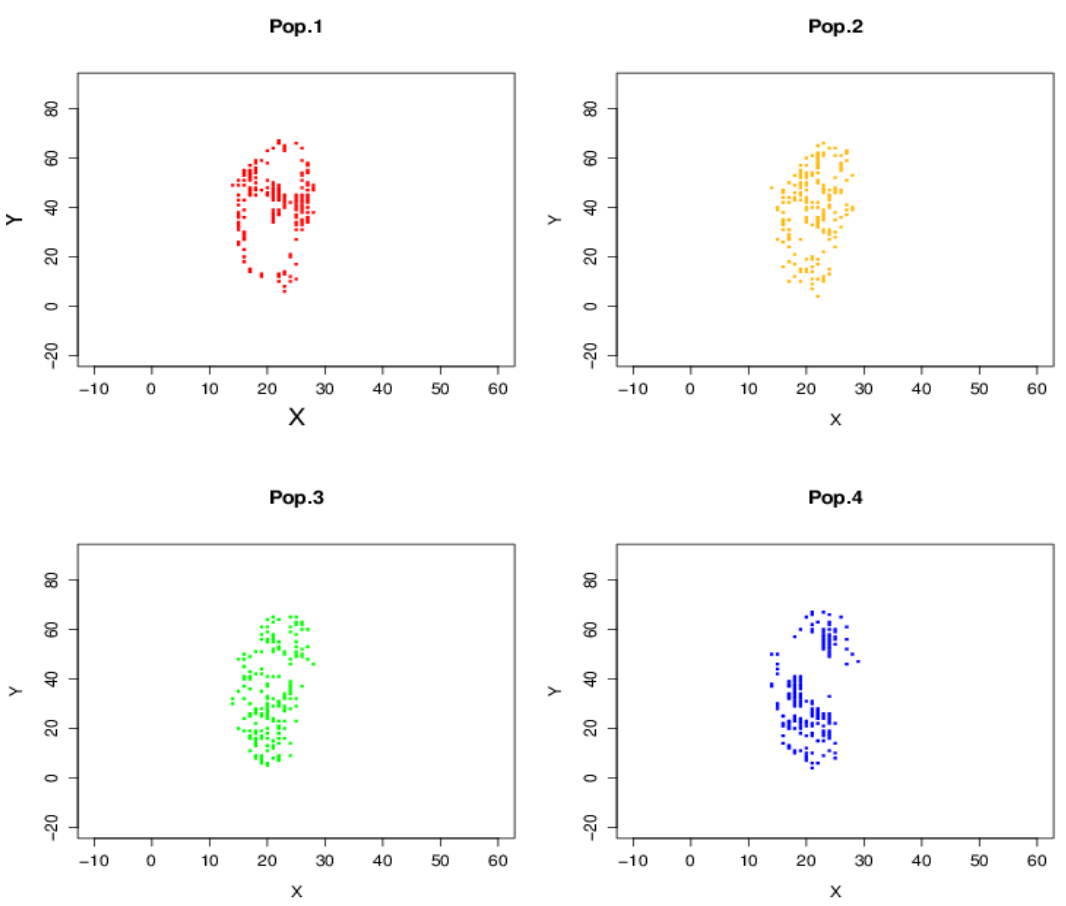

Figura A.29: UGC10331 

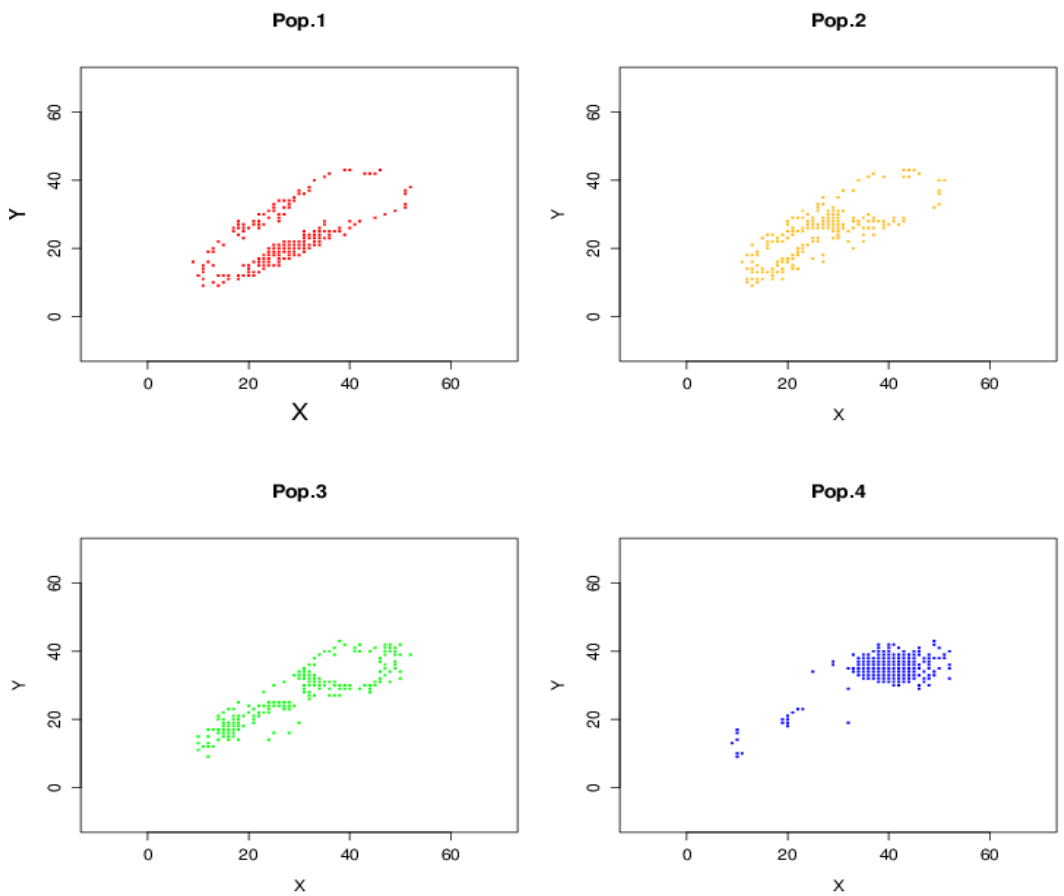

Figura A.30: NGC3991

\section{A.3 Cores e Raios Médios}

A seguir são apresentadas as correlações entre a cor $g-r$ média e o raio normalizado médio de cada população estelar, para as galáxias elípticas (figura A.31), espirais (figura A.32) e espirais tardias (figura A.33). 

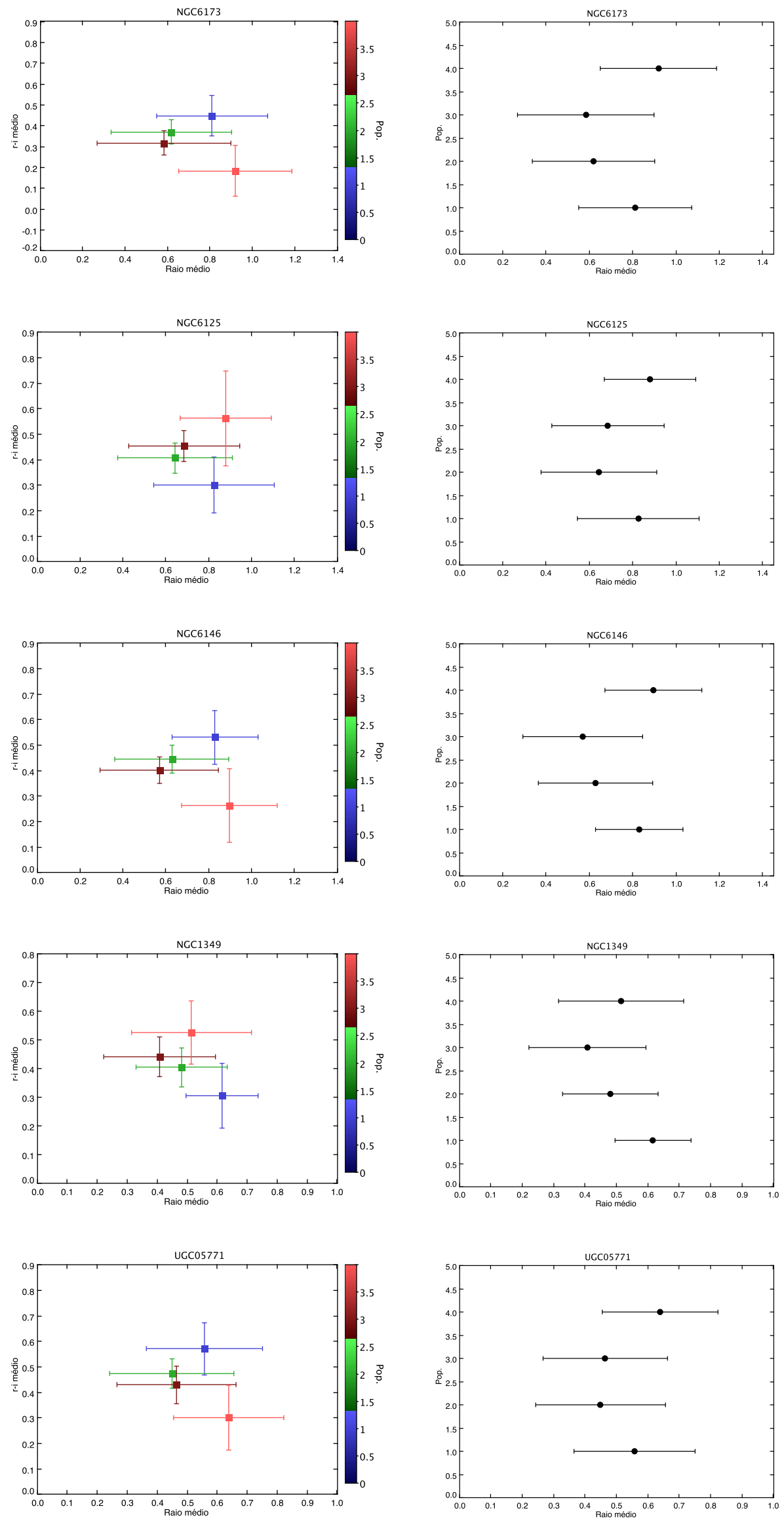

Figura A.31: Raios médios normalizados e a cor g-r em cada população, para as galáxias NGC6173, NGC6125, NGC6146, NGC1349 e UGC05771. 

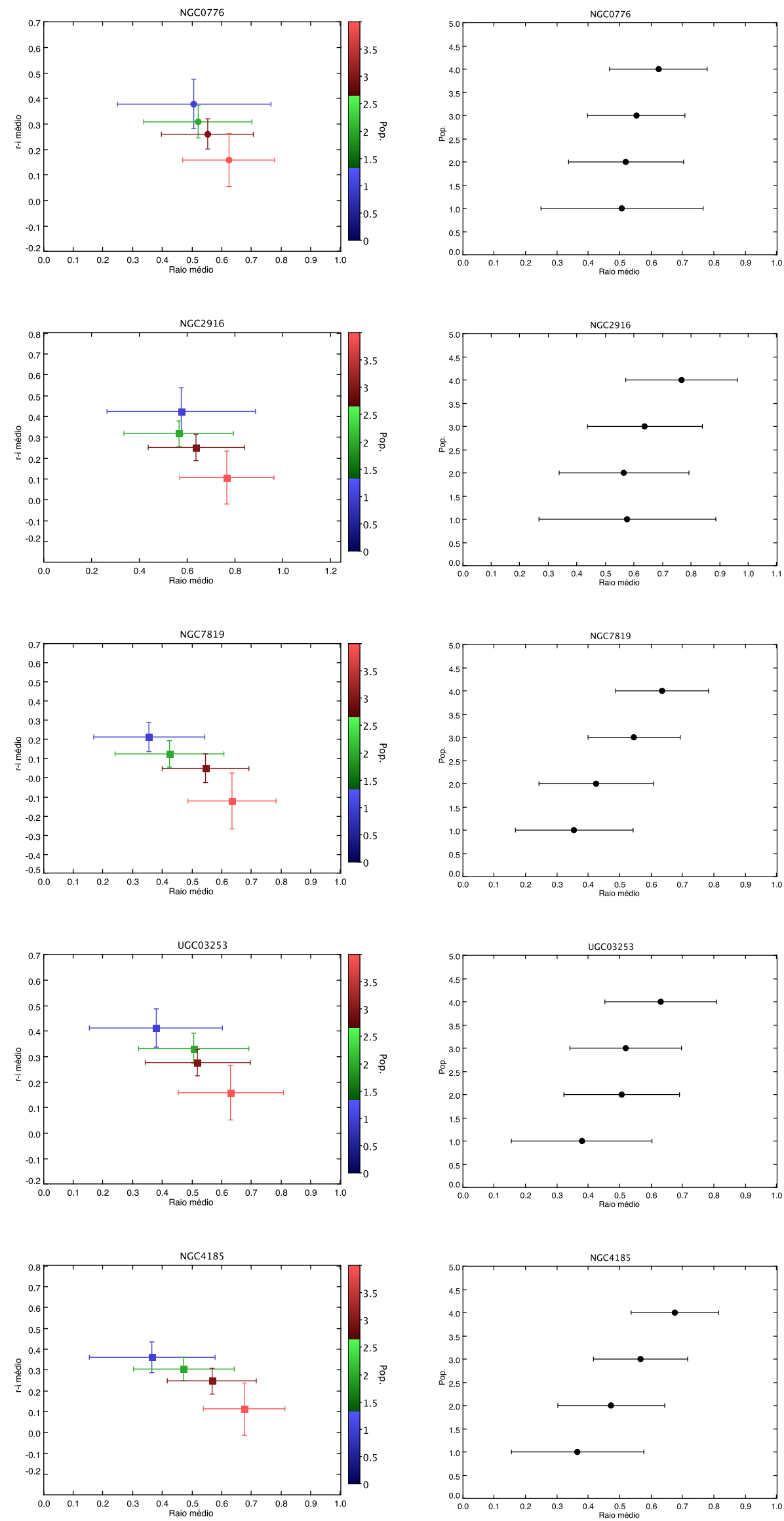

Figura A.32: Raios médios normalizados e a cor g-r em cada população, para as galáxias NGC0776, NGC2916, NGC7819, UGC03253 e NGC4185. 

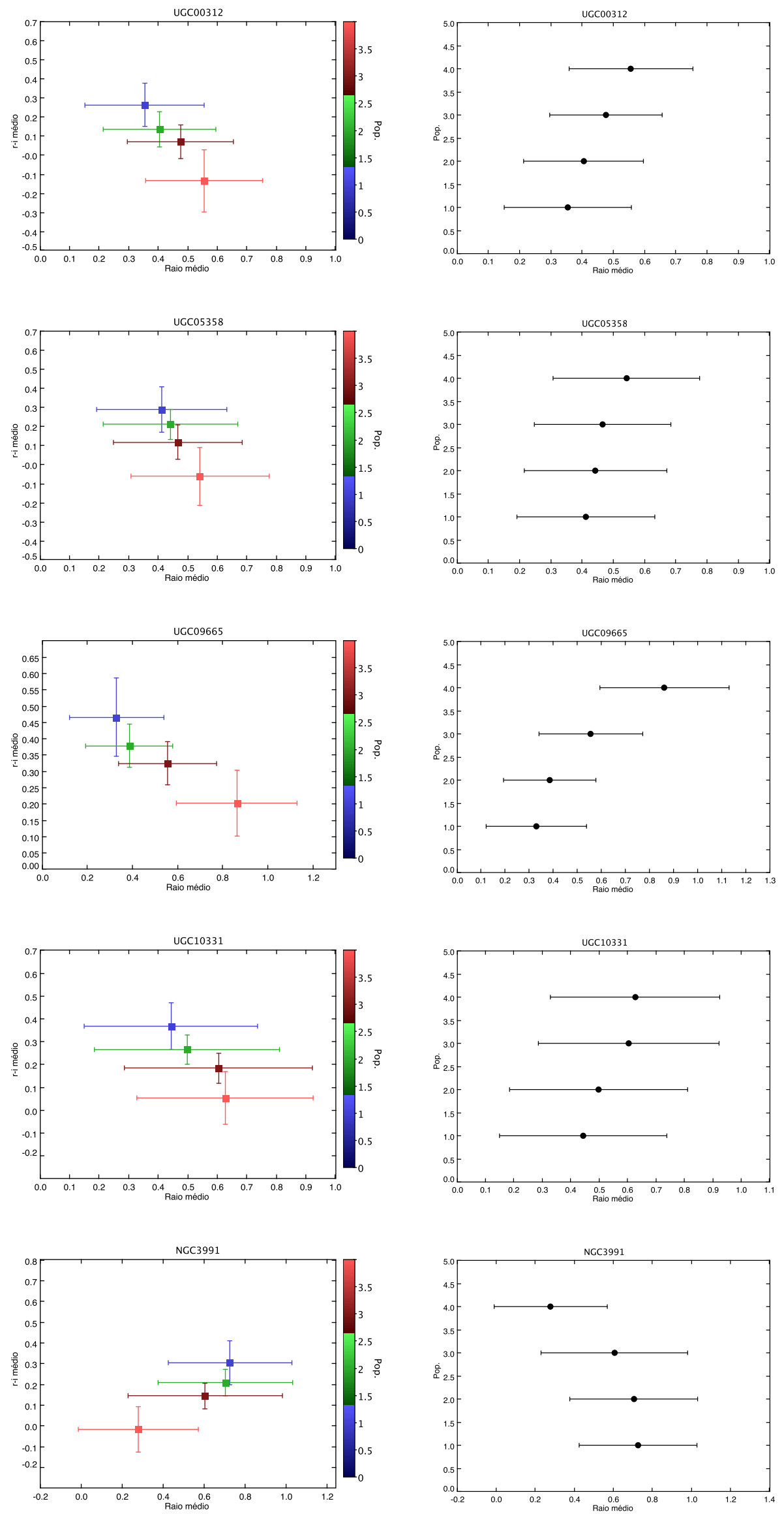

Figura A.33: Raios médios normalizados e a cor g-r em cada população, para as galáxias UGC00312, UGC05358, UGC09665, UGC10331 e NGC3991. 


\section{A.4 Coeficientes de Gini}

A seguir são apresentadas as correlações entre o Coeficientes de Gini e as populações estelares, para as galáxias elípticas (figura A.34), espirais (figura A.35) e espirais tardias (figura A.36).
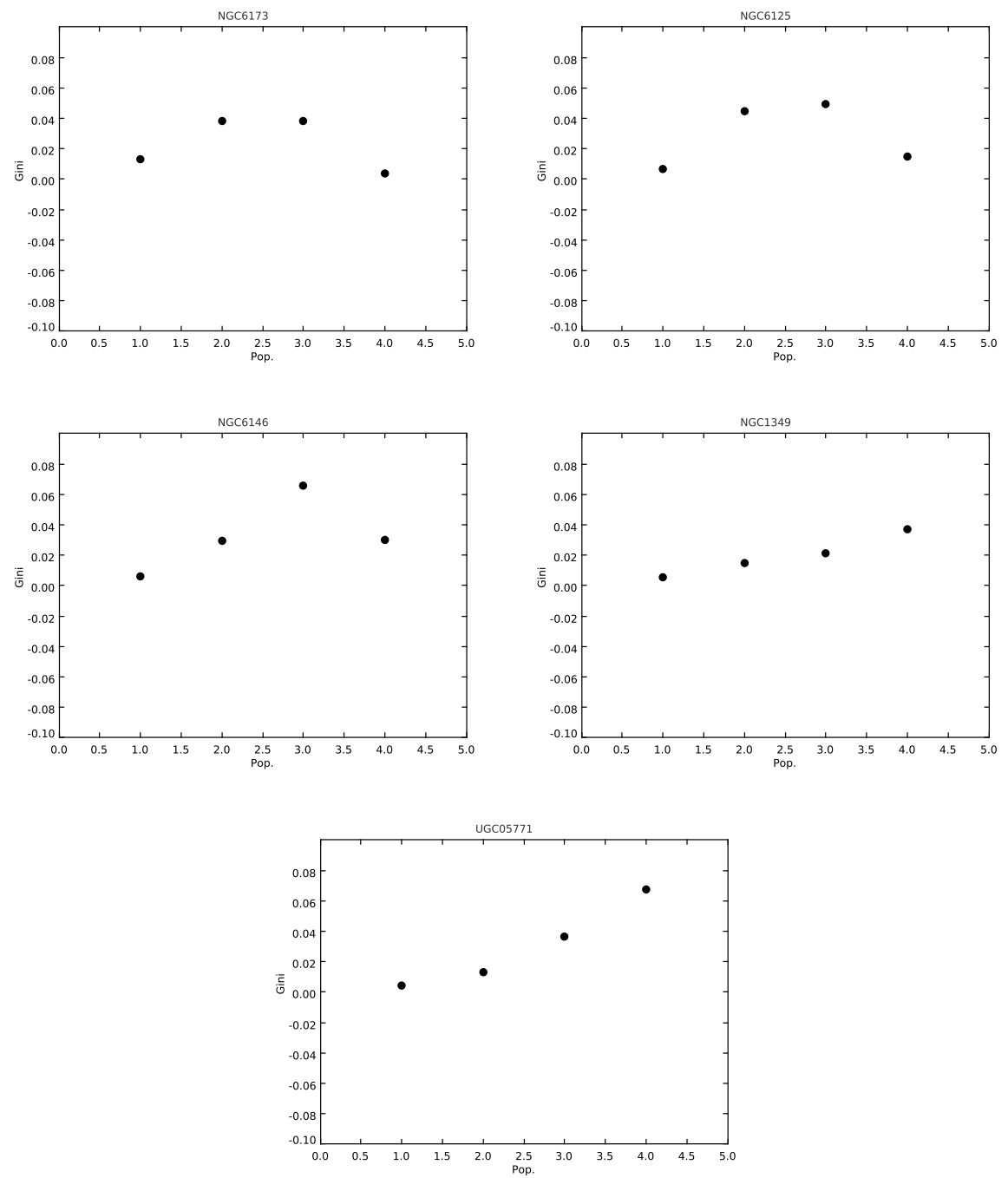

Figura A.34: Coeficiente de Gini para as galáxias NGC6173, NGC6125, NGC6146, NGC1349 e UGC05771. 

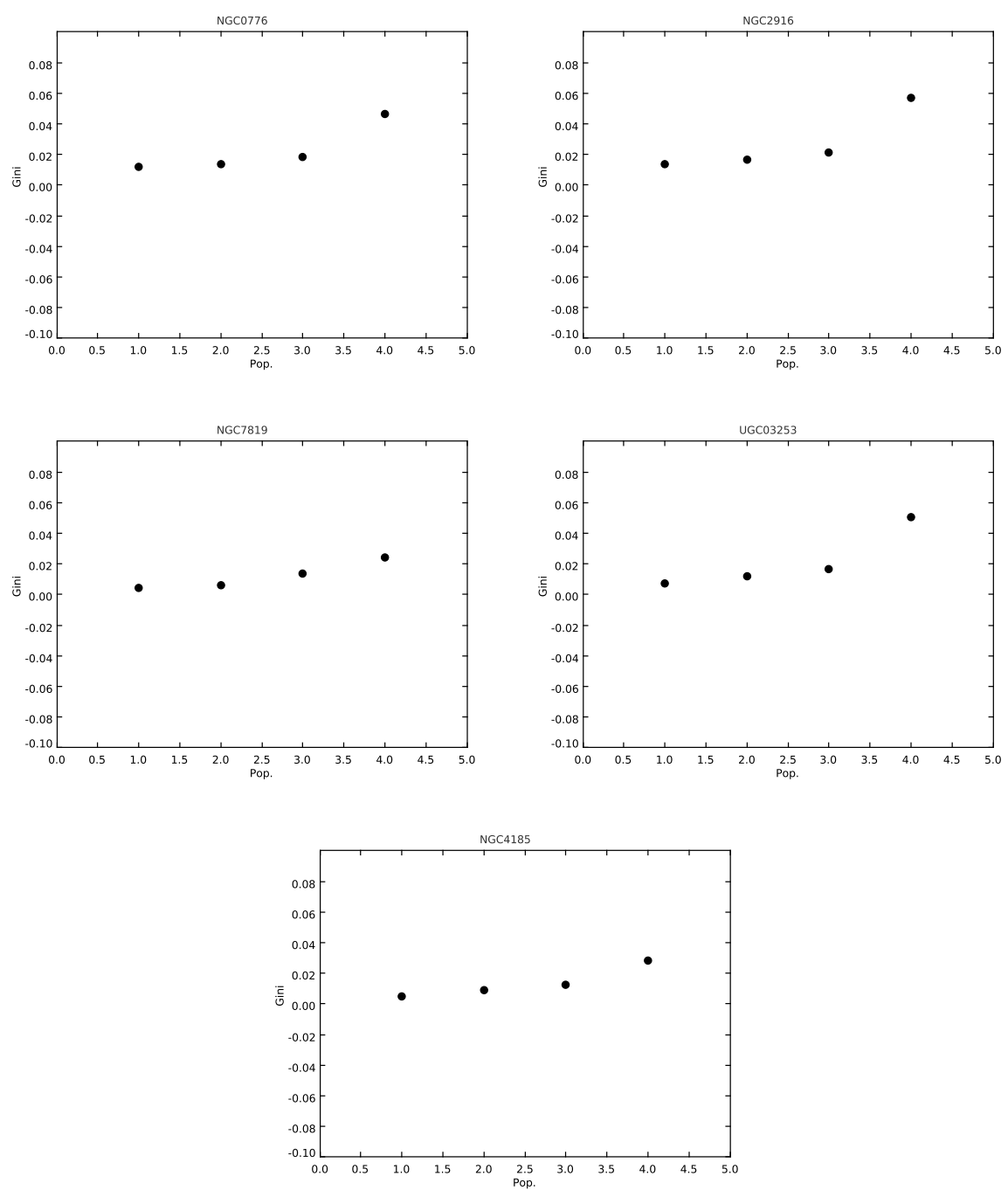

Figura A.35: Coeficiente de Gini para as galáxias NGC0776, NGC2916, NGC7819, UGC03253 e NGC4185. 

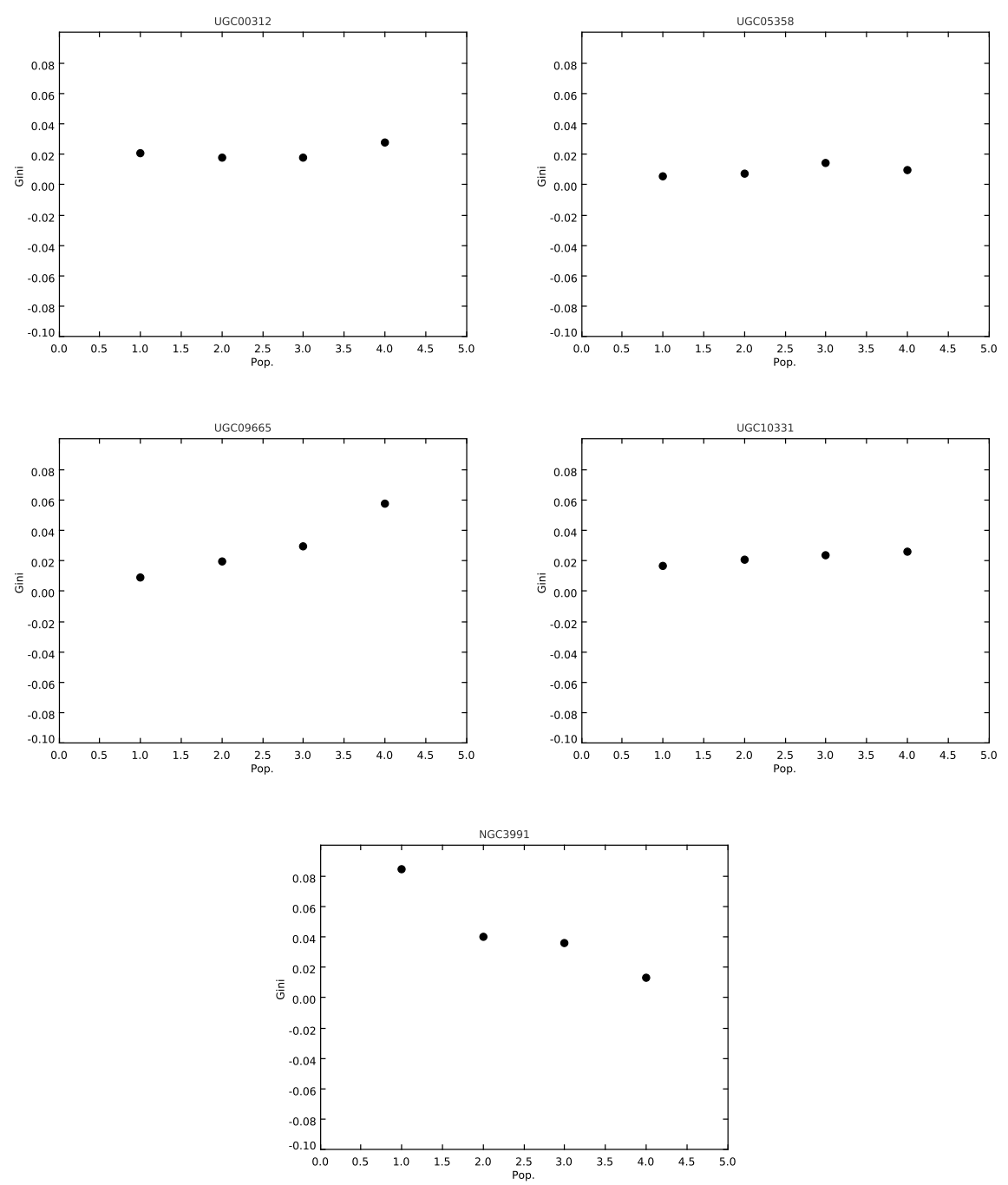

Figura A.36: Coeficiente de Gini para as galáxias UGC00312, UGC05358, UGC09665, UGC10331 e NGC3991.

\section{A.5 Funcionais de Minkowski}

A seguir são apresentadas as correlações entre a Característica de Euler-Poincaré e as populações estelares, para as galáxias elípticas (figura A.37), espirais (figura A.38) e espirais tardias (figura A.39). Nas figuras a esquerda estão representadas a CEP absoluta e a direita a CEP normalizada, de cada galáxia. 

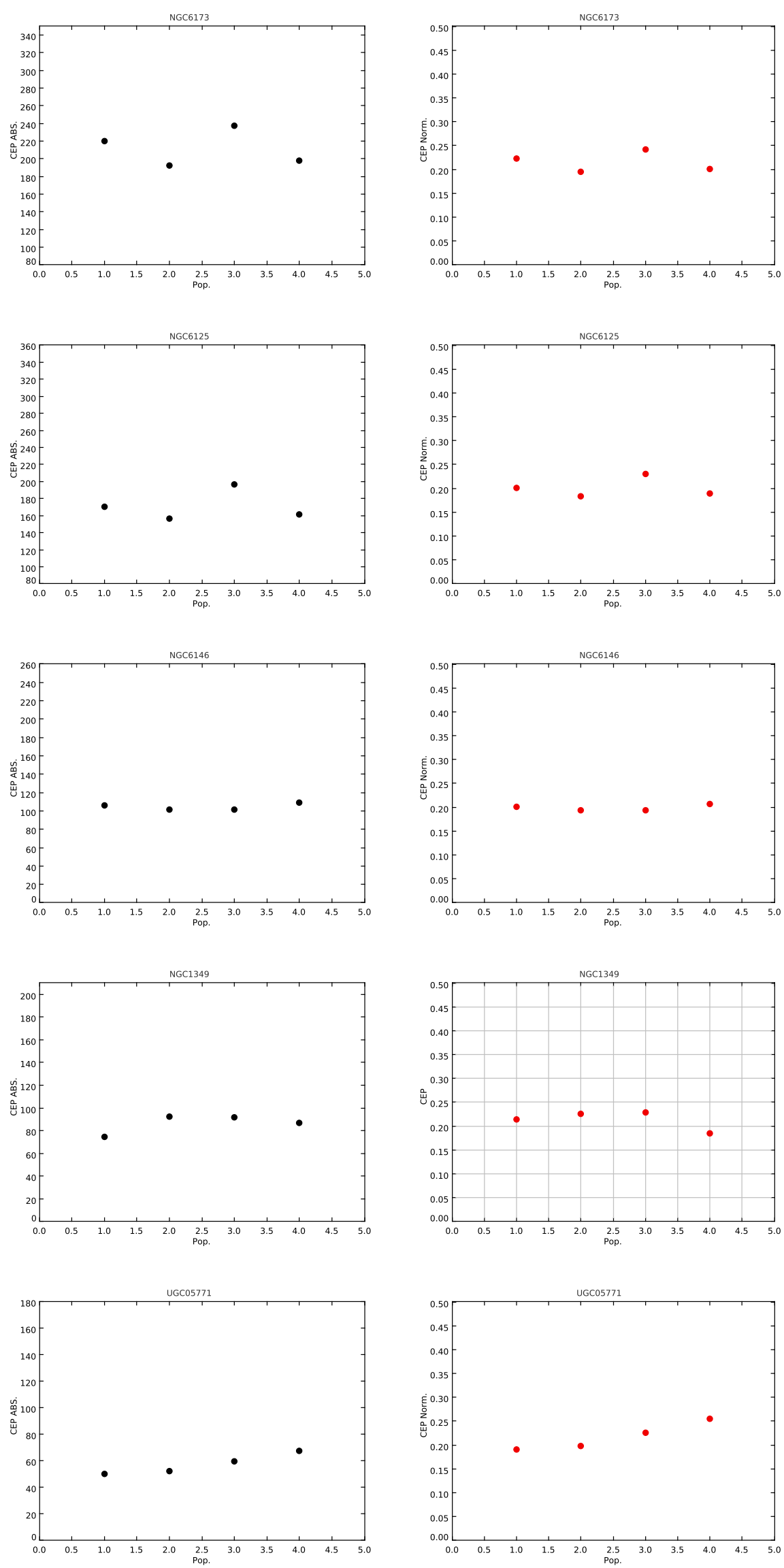

Figura A.37: Característica de Euler-Poincaré, absoluta (pontos pretos) e normalizada (pontos vermelhos), para as galáxias NGC6173, NGC6125, NGC6146, NGC1349 e UGC05771. 

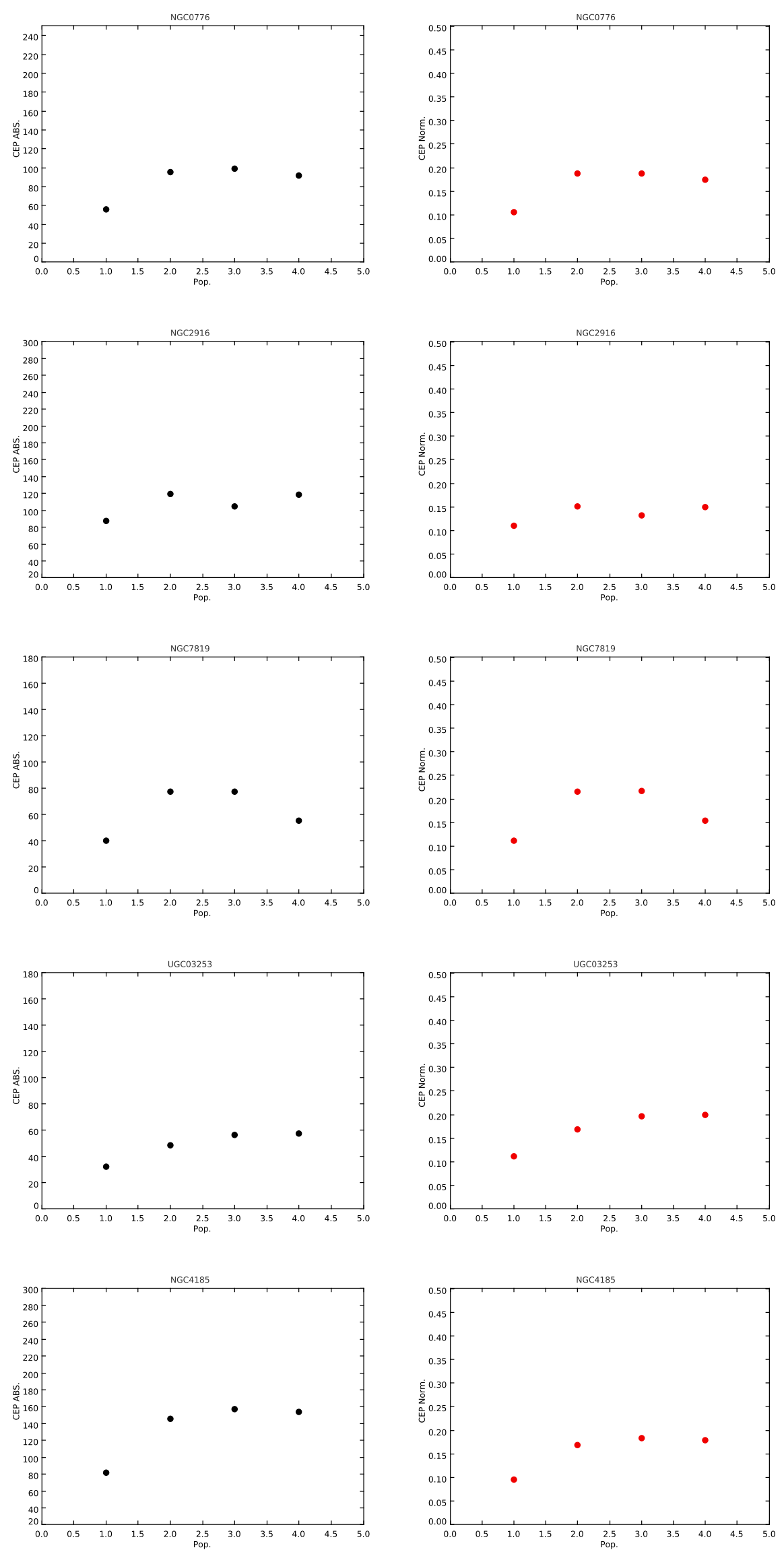

Figura A.38: Característica de Euler-Poincaré, absoluta (pontos pretos) e normalizada (pontos vermelhos), para as galáxias NGC0776, NGC2916, NGC7819, UGC03253 e NGC4185. 

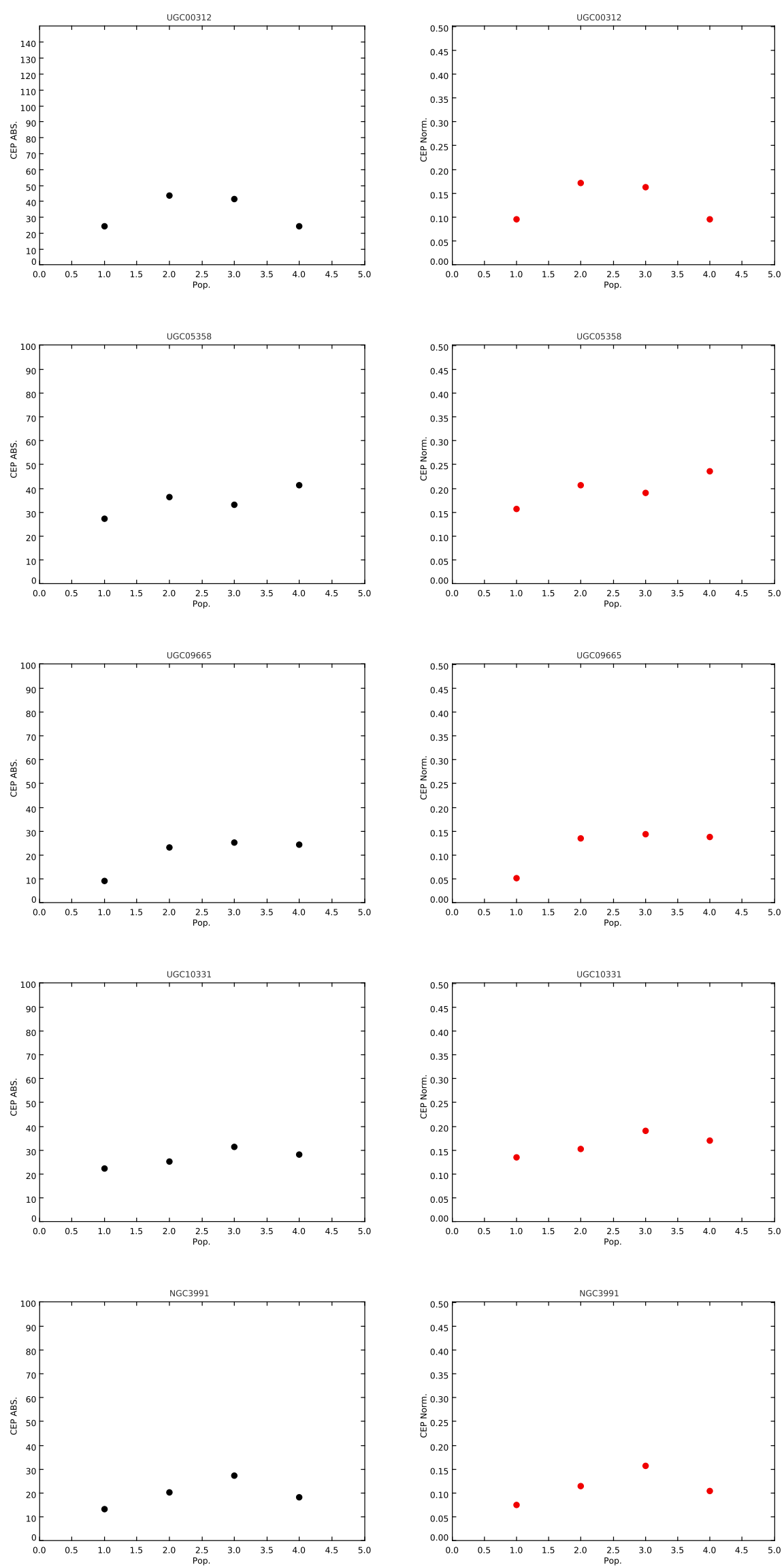

Figura A.39: Característica de Euler-Poincaré, absoluta (pontos pretos) e normalizada (pontos vermelhos), para as galáxias UGC00312, UGC05358, UGC09665, UGC10331 e NGC3991. 